Aus der Klinik für Anästhesiologie

(Prof. Dr. med. M. Quintel)

aus dem Zentrum für Anaesthesiologie, Rettungs- und Intensivmedizin

der Medizinischen Fakultät der Universität Göttingen

\title{
Sympathikusaktivität bei handchirurgischen Operationen mit axillärer Plexus- oder Allgemeinanästhesie
}

\author{
INAUGURAL-DISSERTATION \\ zur Erlangung des Doktorgrades \\ der Medizinischen Fakultät \\ der Georg-August-Universität zu Göttingen \\ vorgelegt von \\ Andreas Manfred Klaholz \\ aus \\ Brilon
}

Göttingen 2013 
Dekan:

I. Berichterstatter:

II. Berichterstatter:

Tag der mündlichen Prüfung: $\quad$ 12.02.2014
Prof. Dr. rer. nat. H. K. Kroemer

Prof. Dr. med. J. Hinz

PD Dr. med. C. Dumont 


\section{Inhaltsverzeichnis}

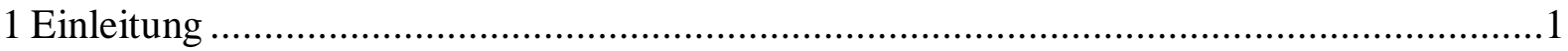

1.1 Anatomie und Physiologie des vegetativen Nervensystems ....................................1

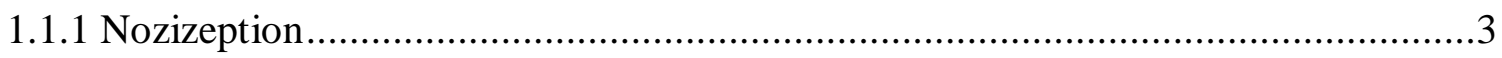

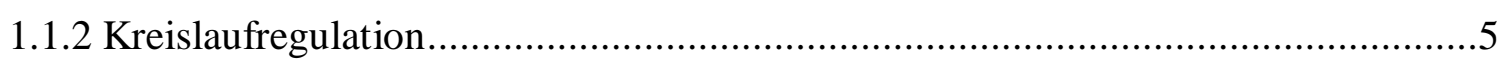

1.1.3 Erhöhte sympathische Aktivität und Stressreaktion ......................................6

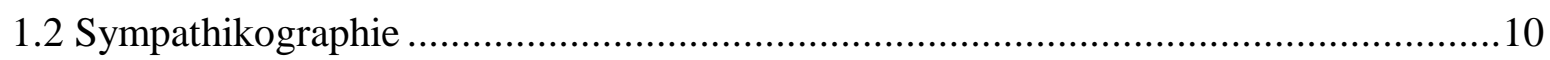

1.2.1 Anatomische und physikalische Eigenschaften der Haut.................................... 14

1.2.2 Physikalische Grundlagen der Sympathikographie .........................................15

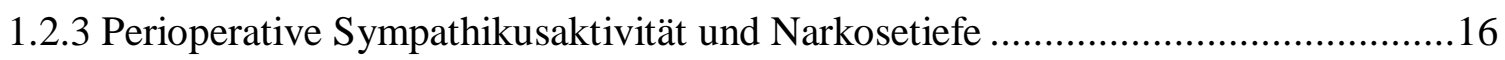

1.2.3.1 EEG, BIS, evozierte Potentiale ........................................................... 17

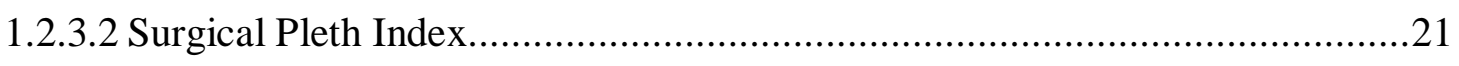

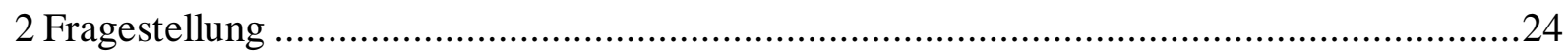

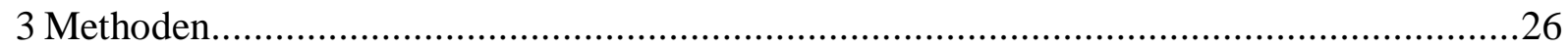

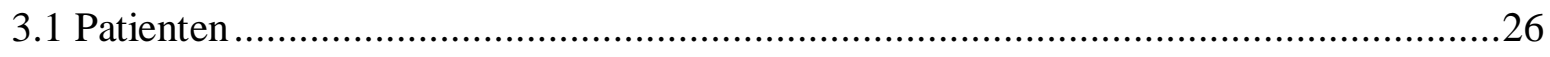

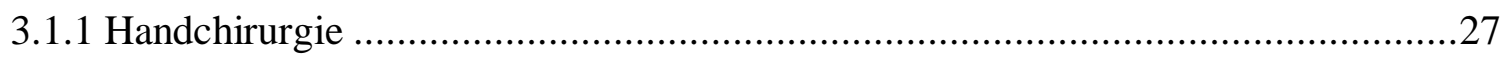

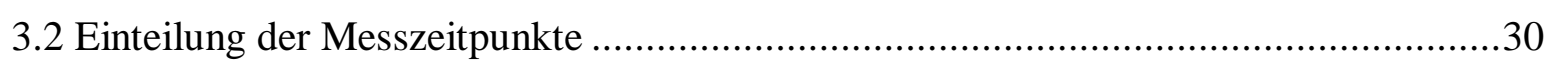

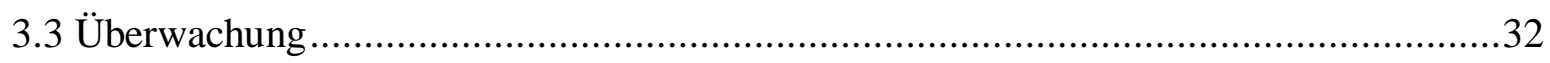

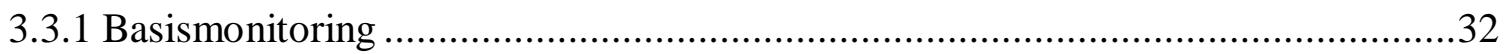

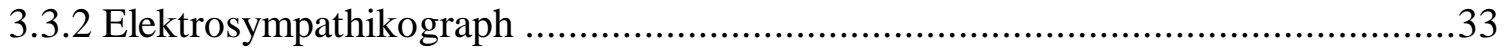

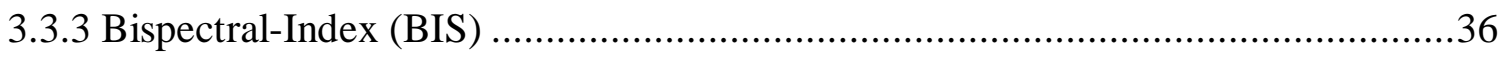

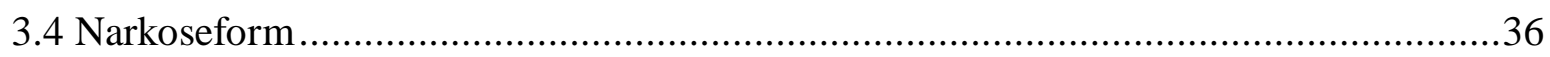

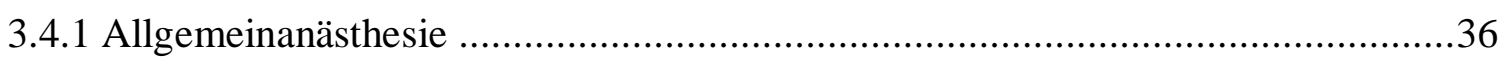

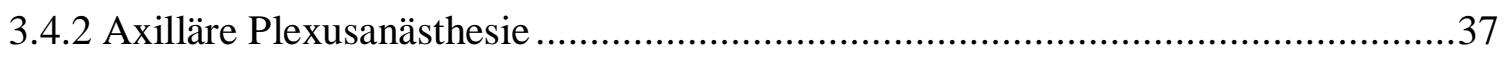

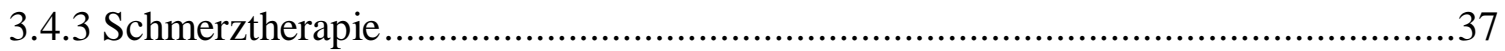

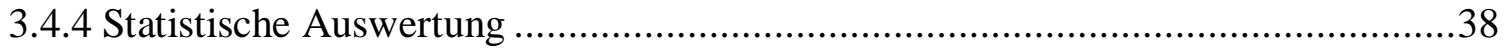

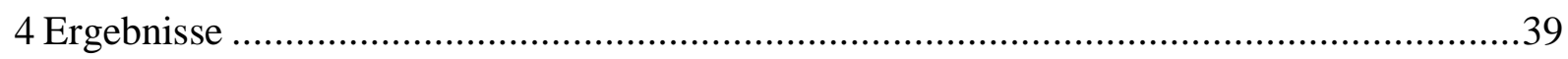

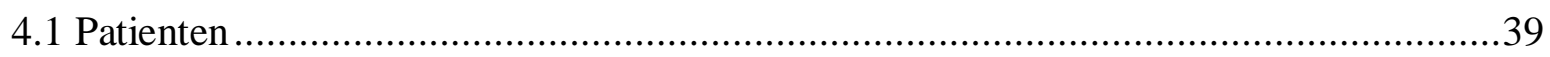

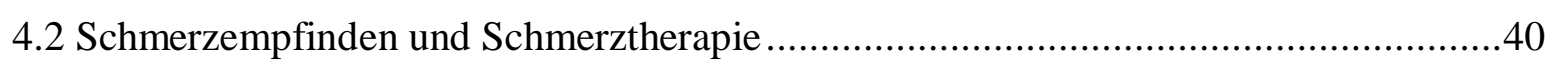

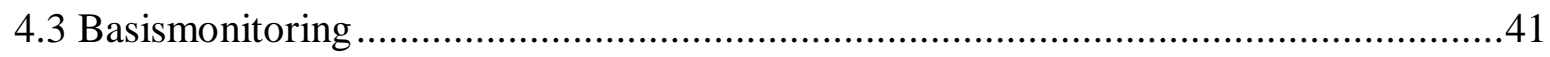

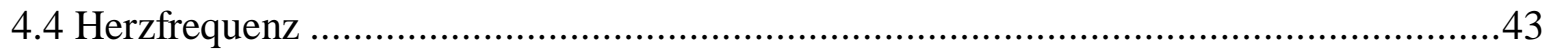

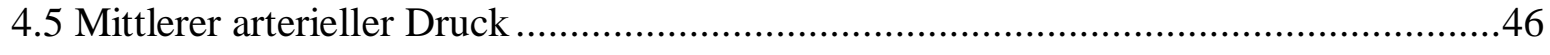

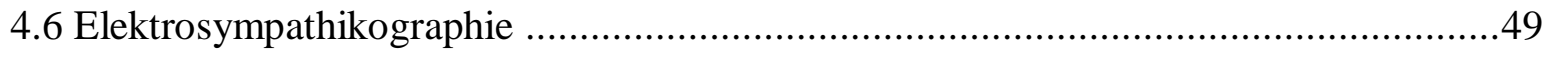

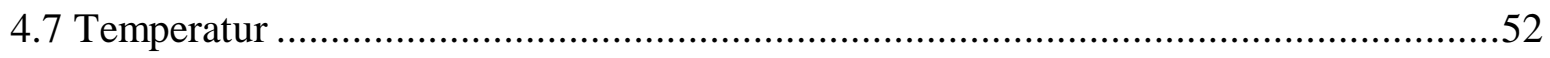

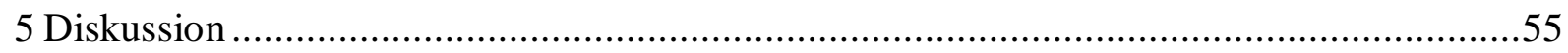

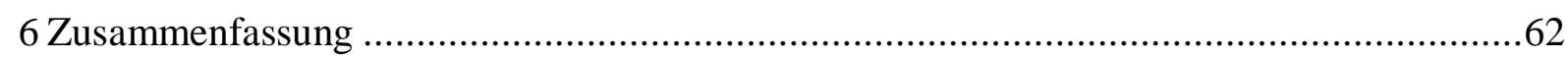

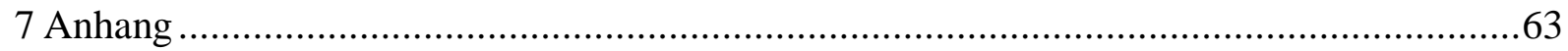

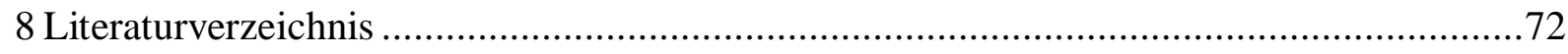




\section{Abkürzungsverzeichnis}

\begin{tabular}{|c|c|}
\hline ACTH & Adrenokortikotropes Hormon \\
\hline $\mathrm{ADH}$ & Antidiuretisches Hormon \\
\hline AEP & akustisch-evozierte Potentiale \\
\hline ASA & American Society of Anesthesiologists \\
\hline AWR & Aufwachraum \\
\hline BIS & Bispectral-Index \\
\hline BMI & Body-Mass-Index \\
\hline $\mathrm{BS}$ & Blutsperre \\
\hline $\mathrm{CO}_{2}$ & Kohlendioxid \\
\hline $\mathrm{CRH}$ & Corticotropin-Releasing Hormon \\
\hline EEG & Elektroenzephalogramm \\
\hline EKG & Elektrokardiogramm \\
\hline ESG & Elektrosympatikograph/Elektrosympathikogramm \\
\hline ETAC & end-tidal anesthetic-agent concentration \\
\hline FFT & Fast-Fourier-Transformation \\
\hline GnRH & Gonadotropin-Releasing Hormon \\
\hline $\mathrm{HBI}_{\text {norm }}$ & normalisiertes Herzschlagintervall \\
\hline $\mathrm{HF}$ & Herzfrequenz \\
\hline ITN & Intubationsnarkose \\
\hline KTS & Karpaltunnelsyndrom \\
\hline MAD & mittlerer arterieller Druck \\
\hline MLAEP & mittel-latente akustisch-evozierte Potentiale \\
\hline NRS & numerische Ratingskala \\
\hline $\mathrm{PPGA}_{\text {norm }}$ & normalisierte Pulswellenamplitude \\
\hline RBS & Ringbandstenose \\
\hline $\mathrm{SaO}_{2}$ & Sauerstoffsättigung \\
\hline SCL & skin conductance level (Hautleitfähigkeitsniveau) \\
\hline SCR & skin conductance response (Hautleitwertsreaktion) \\
\hline SEF & spektrale Eckfrequenz \\
\hline SEP & somatosensorisch-evozierte Potentiale \\
\hline SPI & Surgical Pleth Index \\
\hline SRL & skin resistance level (Hautwiderstandsniveau) \\
\hline
\end{tabular}


SRR skin resistance response (Hautwiderstandsreaktion)

SSI Surgical Stress Index

Temp Temperatur

TIVA total intravenöse Anästhesie

ZNS zentrales Nervensystem 


\section{Einleitung}

\subsection{Anatomie und Physiologie des vegetativen Nervensystems}

Die grundlegenden Funktionen des menschlichen Körpers, deren Regulation unabdingbar für das Leben des einzelnen Individuums ist, werden autonom gesteuert. Die Schaffung der Homöostase, sowie die Anpassung des Organismus an die jeweiligen Umstände und Bedürfnisse sind dem willkürlichen Einfluss entzogen (Jänig 2000). Sie unterliegen der Kontrolle des vegetativen, autonomen Nervensystems. Das vegetative Nervensystem besteht aus einem peripheren und einem zentralen Anteil. Die zentralen Anteile finden sich vor allem im Hirnstamm, Hypothalamus und Großhirn. Die Aktivität des vegetativen Nervensystems kann sowohl durch äußere Reize wie z.B. Schmerzen oder Stress als auch durch körpereigene Signale beeinflusst werden. Zentrales Organ bei der Verarbeitung von Reizen und der damit verbundenen Modulation der Aktivität des vegetativen Nervensystems ist der Thalamus. Er verarbeitet sowohl periphere Signale, die zum Cortex cerebri ziehen, als auch zentrale Signale, die vom Kortex zur Peripherie ausgesandt werden. Der Thalamus wird deshalb auch als „Tor zum Bewusstsein“ bezeichnet (Schiebler et al. 1999c). Der Thalamus ist über auf- und absteigende Bahnen mit dem Hypothalamus, dem wichtigsten Organ bei der Steuerung des vegetativen Nervensystems, verbunden. Der Hypothalamus ist für die Homöostase im menschlichen Körper verantwortlich. Ihm obliegt die Integration der Signale unter anderem von Thalamus und limbischem System (Jänig 2000). Während über den Thalamus sensorische Reize Einfluss auf die Funktion des vegetativen Nervensystems nehmen, wirken Emotionen und affektives Verhalten über das limbische System auf die sympathischen und parasympathischen Reaktionen (Hick 2000). Die Aufgabe der Signalintegration wird vor allem im lateralen Anteil des Hypothalamus wahrgenommen, der im weiteren Verlauf der Reizverarbeitung über die Formatio reticularis Einfluss auf das vegetative Nervensystem nimmt. Der mediale Anteil des Hypothalamus, der wechselseitig mit dem lateralen Hypothalamus in Verbindung steht, analysiert über spezielle Neurone wichtige Stellgrößen wie die Temperatur des Blutes, den Salz- und Zuckerspiegel, sowie den Hormonstatus. Er sendet absteigende Bahnen zur Hypophyse, über die er Einfluss auf periphere Organsysteme nimmt. Die Verbindung zur Neurohypophyse ist nerval, die zur Adenohypophyse hormonal (Jänig 2000). In der Peripherie lassen sich drei Anteile des vegetativen Nervensystems unterscheiden: Sympathikus, Parasympathikus und enterisches Nervensystem (Jänig 2000). Der Sympathikus hat seinen Ursprung in den 
thorakolumbalen Segmenten C8-L1 des Rückenmarks und wird deshalb auch als thorakolumbales System bezeichnet. Die Ursprünge des Parasympathikus liegen im Hirnstamm und im Sakralmark. Er wird deshalb auch als kraniosakrales System betitelt (Hick 2000). Im Magen-Darm-Trakt befindet sich das enterische Nervensystem, welches unabhängig von extrinsischen Einflüssen des Rückenmarks oder des Hirnstamms arbeitet. Die präganglionären Nervenfasern des Sympathikus ziehen von ihrem Ursprung im thorakolumbalen Rückenmark zu den paarig angelegten paravertebralen Ganglien und zu den prävertebralen abdominal gelegenen Ganglien (Ganglion coeliacum, Ganglion mesentericum superius und inferius, Plexus hypogastricus superius), die einzeln angelegt sind. Die paravertebral gelegenen Ganglien bilden an der jeweiligen Seite gemeinsam je einen Grenzstrang, der parallel der Wirbelsäule von zervikal bis sakral verläuft. Vom Grenzstrang bzw. von den abdominal gelegenen Ganglien ziehen die postganglionären Fasern zu den jeweiligen Zielorganen. Wesentliche Zielorgane des Sympathikus sind die glatte Muskulatur sämtlicher Organe, u.a. der Gefäße, der Lunge und der Pupillen. Der Herzmuskel und exokrine Drüsen wie Schweiß- oder Speicheldrüsen werden ebenfalls durch den Sympathikus innerviert (Jänig 2000). Der Ablauf der Signalübertragung von Sympathikus und Parasympathikus unterscheidet sich in einigen wesentlichen Punkten. So erfolgt beim Parasympathikus die Umschaltung der präganglionären Fasern auf postganglionäre Neurone im Wesentlichen erst an oder in den Zielorganen und nicht in den vorgelagerten Ganglien. Wichtige Zielorgane des Parasympathikus sind u.a. die Vorhöfe des Herzens, die glatte Muskulatur sowie die Drüsen des Gastrointestinaltrakts und der Lunge. Die glatte Muskulatur der arteriellen Gefäße wird mit Ausnahme der Gefäße der Geschlechtsorgane und des Gehirns nicht parasympathisch innerviert (Jänig 2000).

Die Transmitter des vegetativen Nervensystems sind Azetylcholin, Noradrenalin und Adrenalin. Azetylcholin und Noradrenalin vermitteln die Signalübertragung an den Synapsen der jeweiligen Nervenenden. Adrenalin dient als Überträgerstoff im ZNS. Die Signalübertragung an allen Endigungen präganglionärer Nervenfasern wird durch die Sekretion von Azetylcholin vermittelt. Azetylcholin ist ebenfalls Überträgerstoff an den meisten postganglionären Fasern des Parasympathikus, während die Signalübertragung an den sympathischen postganglionären Nervenenden durch Noradrenalin erfolgt. Der Effekt des Sympathikus an den Schweißdrüsen wird jedoch über Azetylcholin vermittelt. (Jänig 2000). Die Mehrheit der Zielorgane wird sowohl sympathisch als auch parasympathisch innerviert. Die Wirkungen beider Systeme sind durch die 
physiologischen Regelmechanismen meist entgegengesetzt. Eine Steigerung der Herzleistung bei gesteigerter Sympathikusaktivität bewirkt zugleich eine Abnahme der Aktivität des Gastrointestinaltrakts. Eine gesteigerte Parasympathikusaktivität führt hingegen zum gegenteiligen Effekt. Die Herzleistung wird vermindert und die Aktivität des Gastrointestinaltrakts steigt. Die Harnblase und die Speicheldrüsen stehen beispielhaft für die Tatsache, dass unter ausgeglichenen Bedingungen bei den meisten Organen, die parasympathisch und sympathisch innerviert werden, der Einfluss des Parasympathikus überwiegt. Es gibt aber auch Organe, die nur von dem einen oder dem anderen System innerviert werden. So werden u.a. die ekkrinen Schweißdrüsen nur sympathisch innerviert (Hick 2000, Jänig 2000). Das Zusammenspiel von Sympathikus und Parasympathikus ist die Grundlage für die Homöostase des menschlichen Organismus. Dieses System aus Spieler und Gegenspieler ermöglicht dem menschlichen Körper eine unmittelbare Anpassung an die unterschiedlichsten äußeren Einflüsse und Belastungen. Je nach Anforderung überwiegt die Aktivität des Sympathikus oder des Parasympathikus. Der Parasympathikus ist vor allem in Ruhephasen aktiv und dominiert gegenüber dem Sympathikus, was sich unter anderem in einer Abnahme der Herzfrequenz oder einer Erniedrigung des Blutdrucks bemerkbar macht, dies dient vor allem der Energieerhaltung und der Schaffung von Reserven (Larsen 2010a). Ist hingegen eine erhöhte körperliche Leistung erforderlich, so überwiegt die Aktivität des Sympathikus (Kahle 2001). Die Aktivierung des Sympathikus spiegelt die Anpassung des menschlichen Körpers an die unterschiedlichsten Stressoren dar, die auf den Körper einwirken. Zu diesen Stressoren gehören auch die Einflüsse, denen die Patienten während ihres Krankenhausaufenthaltes ausgesetzt sind. Die Steigerung der Sympathikusaktivität äußert sich direkt wahrnehmbar z.B. in einer Steigerung der Herzfrequenz oder einer Zunahme des Blutdrucks. Gerade in der perioperativen Phase sind die Patienten den unterschiedlichsten Eindrücken ausgesetzt, die als Stressoren empfunden werden können. Diese Stressoren reichen von Empfindungen, die direkt wahrgenommen werden, wie zum Beispiel die ungewohnte oder gar unangenehme Umgebung des Krankenhauses, bis zu den Schmerzreizen, die während einer Operation unbewusst wahrgenommen werden.

\subsubsection{Nozizeption}

Die Integration von nozizeptorischen Reizen stellt einen wichtigen Faktor bei der perioperativen Sympathikusaktivierung dar. Die Verarbeitung von Schmerzreizen und die daraus resultierenden Reaktionen, unter anderem des vegetativen Nervensystems, 
beruhen auf einer komplexen Verschaltung im neuronalen Netzwerk. Frei endende Nervenfasern, so genannte Nozizeptoren, dienen als periphere Empfänger des Schmerzreizes. Als schmerzleitende Fasern werden marklose C-Fasern von markhaltigen A $\delta$-Fasern unterschieden. Die Perikaryen der Nervenfasern befinden sich im Spinalganglion. Die Fasern unterscheiden sich in der Leitungsgeschwindigkeit und in der verarbeitenden Schmerzqualität. Die A $\delta$-Fasern leiten scharfe, stechende Schmerzempfindungen besonders schnell und ermöglichen über eine Verschaltung mit Motoneuronen auf Rückenmarksebene eine direkte reflexartige motorische Reaktion. So wird z.B. ein Fuß beim Tritt in eine Scherbe reflexartig zurückgezogen (Schaible und Schmidt 2000). Die C-Fasern vermitteln die Empfindung von diffusen, dumpfen oder ziehenden Schmerzen. Die Schmerzleitung ist wesentlich langsamer als bei den A $\delta$-Fasern und der Schmerz ist wesentlich schlechter lokalisierbar (Bromm 2002). Die Schmerzfasern enden im Hinterhorn des Rückenmarks. Dort findet die synaptische Umschaltung vom ersten auf das zweite Neuron statt und die Fasern kreuzen anschließend auf die Gegenseite. Im anterolateralen System des Rückmarks werden die Schmerzsignale in Tractus (Schmerzbahnen) zentral zum Thalamus, zur Formatio reticularis, zum limbischen System und zum Mesenzephalon weitergeleitet. Außerhalb des anterolateralen Systems, das den Großteil der Schmerzleitung übernimmt, wird u.a. die Eingeweidesensibilität über den Hinterstrang zentralwärts geleitet (Lorke 2002). Im Gehirn dient vor allem der Thalamus mit seinen Kerngebieten als Umschaltstation, bevor die Schmerzsignale zur endgültigen Verarbeitung in die entsprechenden Areale des Kortex gelangen. Entlang der gesamten Schmerzleitung finden sich Verschaltungen mit dem vegetativen Nervensystem (Brodner et al. 2011). Bei der Verarbeitung von Schmerzsignalen werden eingehende Signale auf Rückenmarksebene über absteigende Bahnen supraspinaler Neurone gehemmt bzw. moduliert. Der Körper kann sich so gewissermaßen vor überschießenden Schmerzsignalen schützen (Schaible und Schmidt 2000). Jede Form von Gewebeschädigung kann direkt beispielsweise durch Druck, Hitze oder Kälte aber auch indirekt durch die Substanzen, die bei einem Zellschaden freigesetzt werden, zu einer Reizung der Nozizeptoren und damit zu einer Schmerzempfindung führen. Die ausgelöste Schmerzreaktion führt dann über die Reizung des vegetativen Nervensystems zu einer Aktivierung des Sympathikus. Daraus resultieren beispielsweise eine Erhöhung des Blutflusses in der Skelettmuskulatur bei gleichzeitiger Herabsetzung der Durchblutung von Haut und Gastrointestinaltrakt. Weiterhin wird das 
Herzzeitvolumen erhöht, das Nebennierenmark zur Katecholaminausschüttung angeregt und die Schweißdrüsen aktiviert (Schaible und Schmidt 2000).

\subsubsection{Kreislaufregulation}

Eine Zunahme der Sympathikusaktivität geht unter anderem mit einer Stimulation des Herz-Kreislauf-Systems einher. Die Steigerung der kardiozirkulatorischen Aktivität erfolgt durch ein komplexes Zusammenspiel von myogenen, metabolischen, hormonalen und nervalen Effekten. Die Aktivität des Sympathikus oder des Parasympathikus ermöglicht über verschiedene Stellgrößen wie z.B. Herzleistung, peripherer Gefäßwiderstand oder Hormonausschüttung aus dem Nebennierenmark die Anpassung des Blutkreislaufs an die unterschiedlichsten Bedürfnisse. Bei der bedarfsgerechten Verteilung des vorhandenen Blutvolumens und der kurzfristigen Blutdruckregulation hat das vegetative Nervensystem einen wesentlichen Anteil (HachWunderle 2004). Die Zunahme der Sympathikusaktivität führt u.a. über $\beta$-Rezeptoren zu einer Steigerung der Herzfrequenz und der Kontraktionskraft des Herzens. Über $\alpha$ Rezeptoren in den Gefäßwänden der Widerstandsgefäße wird eine Gefäßkontraktion vermittelt (Jänig 2000). Die gesteigerte Sympathikusaktivität führt ferner zu einer Freisetzung von Adrenalin und Noradrenalin aus dem Nebennierenmark und aus weiteren Paraganglien, die die direkte Sympathikuswirkung weiter verstärkt (Schiebler et al. 1999b). Es kommt zu einer Umverteilung des vorhandenen Blutvolumens. Die Durchblutung in Organsystemen wie der Haut oder des Gastrointestinaltrakts nimmt ab, während die Skelettmuskulatur stärker durchblutet wird (Jänig 2000). Diese Reaktion wird nach dem Physiologen Walter B. Cannon auch als Fight-or-flight-Reaktion bezeichnet (Holst 1993). Neben dieser bedarfsgerechten Verteilung spielt das vegetative Nervensystem eine wesentliche Rolle bei der kurzfristigen Einstellung des arteriellen Blutdrucks. Der arterielle Blutdruck wird durch Barorezeptoren überwacht. Diese Druckrezeptoren, die sich in den Gefäßwänden der großen arteriellen Gefäße befinden, reagieren auf die Dehnung der Gefäßwand, die vom Füllungszustand des Gefäßes abhängig ist. Die Signale der Barorezeptoren werden an die zentralen Regelzentren im Hypothalamus und in der Medulla oblongata weitergeleitet. Den größten Anteil an der Regulation des arteriellen Blutdrucks haben die Barorezeptoren, die sich im Aortenbogen und im Karotissinus befinden (Busse 2000). Die Aktivierung der Barorezeptoren regelt den arteriellen Blutdruck über Pressorezeptorreflexe. Eine Erregung der Barorezeptoren durch die Dehnung der Gefäßwand führt so zu einer Aktivierung von Neuronen im 
Nucleus tractus solitarii, die von dort zu Interneuronen in der Medulla oblongata weitergeleitet wird. Diese Interneurone hemmen nun weitere Neurone in der Medulla, die ihrerseits mit präganglionären Neuronen im Rückenmark verbunden sind. Die Hemmung der Interneurone führt zur Abnahme der Aktivität von Vasokontriktorneuronen der Widerstandsgefäße und von sympathischen Kardiomotoneuronen. Die parasympathischen Kardiomotoneurone hingegen werden aktiviert. Die Gefäße werden weit gestellt und die Herzfrequenz sinkt, was insgesamt zu einem Abfall des arteriellen Drucks führt. Nimmt die Erregung der Barorezeptoren ab, so führt dies zu einer entgegengesetzten Reaktion der Pressorezeptorreflexe (Jänig 2000). Durch diese Komplexizität sind die Kreislaufreaktionen während operativer Eingriffe unter Allgemeinanästhesie oder Regionalanästhesie sehr vielfältig und kaum vorherzusagen (Lipfert und Arndt 1993). Daher sind ein exaktes, kontinuierliches Kreislaufmonitoring und die Erfassung der neurovegetativen Funktionszustände wünschenswert, um auf kritische Veränderungen des Herz-Kreislauf-Systems frühzeitig reagieren zu können.

\subsubsection{Erhöhte sympathische Aktivität und Stressreaktion}

Perioperativ ist der Patient den unterschiedlichsten Einflüssen bzw. Stressoren ausgesetzt. Diese reichen von Emotionen wie Angst und Panik über die chirurgisch ausgelösten Reize bis zu möglichen intraoperativen und postoperativen Schmerzen. Diese Reize, wie beispielsweise oben bei der Verarbeitung von Schmerzreizen beschrieben, lösen im menschlichen Körper eine zunehmende Aktivität des Sympathikus aus, die eine Reihe von physiologischen Kaskaden zur Folge hat. Hierbei spielen die SympathikusNebenierenmark-Achse und die Hypothalamus-Hypophysen-Nebennierenrinden-Achse eine zentrale Rolle (Tolksdorf 1985, Tsigos und Chrousos 2002). Insgesamt wird diese Reaktion auch als Stressreaktion bezeichnet. Das wichtigste Organ bei der Verarbeitung äußerer Reize und der damit verbundenen Steigerung der Aktivität des Sympathikus ist der Hypothalamus. Die gesteigerte Sympathikusaktivität hat zum einen direkten Einfluss auf die sympathisch innervierten Organe, so wird u.a. durch B-Rezeptoren die Herzleistung gesteigert (s.o.). Zum anderen führt die vermehrte Aktivierung des Sympathikus zu einer neuronal vermittelten und durch cholinerge Synapsen gesteuerten Freisetzung der katabolen Hormone Adrenalin und Noradrenalin aus dem Nebenierenmark. Durch diese Hormone kommt es indirekt zu einer Verstärkung der bereits direkt ausgelösten sympathischen Effekte. Diese Kaskade wird als SympathikusNebenierenmark-Achse oder sympathikoadrenales System bezeichnet (Anand und Hickey 
1992, Winterhalter 2007). Die Folge der Ausschüttung von Adrenalin und Noradrenalin ist ein Anstieg der Herzleistung und des Blutdrucks. Die Durchblutung der Muskulatur und des Gehirns nimmt auf Kosten von Haut und Eingeweiden zu (Tolksdorf 1985, Tsigos und Chrousos 2002). Um dem Körper mehr Energie bereitzustellen, führt die steigende sympathische Aktivität über $\beta_{2}$-Rezeptoren zu einer Steigerung der Glykogenolyse in der Leber. Die Muskulatur wird aktiviert und der Blutzuckerspiegel steigt. Zentrales Steuerorgan der Hypothalamus-Hypophysen-Nebennierenrinden-Achse und der endokrinen Stressreaktion ist der Hypothalamus. Seine Aktivität wird durch zentrale Regionen des Amygdala-Hippokampus-Komplexes, des Nulceus arcuatus und des mesolimbischen System moduliert (Tsigos und Chrousos 2002). Releasinghormone werden aus den hypothalamen Kerngebieten des Nucleus paraventricularis freigesetzt. Bei der Vermittlung der erhöhten sympathischen Aktivität haben vor allem das CorticotropinReleasing Hormon (CRH) und das antidiuretische Hormon (ADH) eine große Bedeutung. Durch die Wirkung von CRH und ADH wird Adrenokortikotropin (ACTH) aus dem Hypophysenvorderlappen, der Adenohypophyse, freigesetzt (Tsigos und Chrousos 2002). ACTH regt die Zellen der Nebennierenrinde zu vermehrter Cortisolproduktion und -sekretion an. Der Cortisolspiegel hemmt im Sinne einer Rückkopplung die weitere Freisetzung von $\mathrm{CRH}$ und $\mathrm{ADH}$ aus dem Hypothalamus und von ACTH aus der Hypophyse (Wuttke 2000). Die Wirkung des freigesetzten Cortisols ist vielfältig. Cortisol zeigt u.a. eine permissive Wirkung im Zusammenspiel mit den ausgeschütteten Katecholaminen. So ist die katecholaminabhängige Durchblutungsförderung der Skelettmuskulatur, die mit einer Minderdurchblutung der glatten Muskulatur des Intestinaltraktes und der Haut einhergeht, nur im Zusammenspiel mit Cortisol möglich. Um die Bereitstellung der benötigten Energie während der Stressreakton sicherzustellen, kommt es durch Cortisol zu einer Steigerung der Gluconeogenese der Leber, sowie zu einem vermehrten Muskelabbau. Der Abbau von Muskelproteinen setzt Aminosäuren frei, die zu Glucose verstoffwechselt werden können. Neben dieser eiweißkatabolen Wirkung hat Cortisol noch eine antianabole Wirkung. Cortisolbedingt stehen weniger Aminosäuren zur Verfügung, die zum Muskelaufbau verwendet werden können. Des Weiteren wird die Lipolyse gesteigert und die Liponeogenese gehemmt. Neben der Funktion der Energiebeschaffung hat Cortisol zudem eine antiinflammatorische und immunsuppressive Wirkung (Wuttke 2000).

So sehr die Abläufe der Stressreaktion der Homöostase des Körpers dienlich sind, so sehr kann eine zu große oder chronisch überhöhte Sympathikusaktivierung die Homöostase 
beeinträchtigen. Bei den Untersuchungen der Folgen massiver oder chronischer Sympathikusaktivität konnten erhebliche negative Einflüsse unter anderem auf das Immunsystem, die Fertilität und das Herz-Kreislaufsystem festgestellt werden (Brodner et al. 2011, Tsigos und Chrousos 2002). Die Immunantwort des Körpers auf einen chirurgischen oder infektbedingten Reiz führt zur Freisetzung von Zytokinen wie TNF- $\alpha$, Interleukin-6 und Interleukin-1 $\beta$. Diese Entzündungsmediatoren sind jeder für sich oder gemeinsam potente Aktivatoren der Hypothalamus-Hypophysen-NebenierenrindenAchse. Das in der Folge freigesetzte Cortisol wirkt immunsuppressiv (Tsigos und Chrousos 2002). Die über den Sympathikus freigesetzten Katecholamine wirken ebenfalls modulierend auf das Immunsystem. Der Einfluss auf die Zytokinproduktion und die Veränderung der Immunantwort lymphatischer Zellen bewirkt eine Abnahme von proinflammatorisch wirkenden Substanzen und eine Zunahme antiinflammatorischer Mediatoren. Die Immunantwort wird insgesamt abgeschwächt (Elenkov et al. 2000). Es konnte nachgewiesen werden, dass eine verminderte Immunantwort im Zusammenhang mit einer erhöhten - durch einen Tumor verursachten - Morbidität und Mortalität steht (Brodner et al. 2011). Eine chronisch überhöhte Sympathikusaktivierung führt zu einem dauerhaft erhöhten CRH-Spiegel. CRH hemmt ebenso, wie die bei der Stressreaktion freigesetzten Glukokortikoide, die Ausschüttung von GnRH (Gonadotropin-Releasing Hormon) aus den Neuronen des Hypothalamus. Die Hemmung von GnRH hat eine Abnahme der Spermatogenese und des Eizellenwachstums mit Abnahme der Fertilität zur Folge (Tsigos und Chrousos 2002). Ferner bedingt die dauerhafte Steigerung der sympathischen Aktivität pathologische Veränderungen innerhalb des kardiovaskulären Systems. Die Zunahme der Herzleistung kann zu einem Missverhältnis zwischen myokardialem Sauerstoffbedarf und myokardialem Sauerstoffangebot führen. Der prokoagulatorische Einfluss des Sympathikus auf das Gerinnungssystem, durch eine vermehrte Freisetzung von Faktor VIII und von Willebrand Faktor, sowie durch eine Hemmung der Fibrinolyse, erhöht das Risiko myokardialer Schädigungen. Eine veränderte Stabilität der Koronarenplaques und die erhöhten Kräfte, die durch eine gesteigerte Hämodynamik auf die Plaques wirken und diese reißen lassen können, erhöhen die Möglichkeiten eines perioperativen Infarkts zusätzlich (Brodner et al. 2011).

In der klinischen Anästhesie werden die neurovegetativen Regelmechanismen und damit der Funktionszustand des Sympathikus gedämpft. Eine qualitativ gute Anästhesie zeigt sich einerseits durch einen neurovegetativen Funktionszustand ohne totale 
neurovegetative Blockade und ohne das Auftreten von überschießenden Stressreaktionen durch Erhöhung des sympathischen Funktionszustandes andererseits (Götte 1999). 


\subsection{Sympathikographie}

Mit der Sympathikographie bzw. der Hautwiderstandsmessung steht eine Möglichkeit zur Bestimmung der Narkosetiefe zur Verfügung, mit der die Sympathikusaktivität als Indikator für eine ausreichende Sedierung und Analgesie gemessen werden kann. Sie steht im Mittelpunkt der hier durchgeführten Studie. Die grundlegenden wissenschaftlichen Untersuchungen zur elektrodermalen Aktivität - ein Begriff unter dem Johnson und Lubin 1966 die unterschiedlichsten bis dato entdeckten elektrischen Erscheinungen der Haut zusammenfassten - und der damit verbundenen Hautwiderstandmessung, fanden bereits Ende des 19ten Jahrhunderts statt (Bouscein 1988). Die Mitarbeiter des französischen Neurologen Jean-Martin Charcot, Romain Vigouroux und Charles Féré legten mit ihren Forschungsergebnissen den Grundstein in der Erforschung der elektrodermalen Aktivität. Vigouroux publizierte bereits 1879 die Messung des tonischen Hautwiderstandes bei verschiedenen Patientengruppen als mögliches diagnostisches Mittel. Bei seinen Untersuchungen hatte er festgestellt, dass Patienten mit einer Halbseitenlähmung auf der gelähmten Seite einen höheren Hautwiderstand aufwiesen als auf der intakten Seite (Burk 2005, Dawson et al. 2000). Charles Féré legte 1888 an zwei auf der Haut angebrachten Elektroden einen Gleichstrom an und entdeckte eine Änderung des Widerstandes zwischen den Elektroden, sobald er die Probanden unterschiedlichsten Sinneswahrnehmungen aussetzte (Féré 1888). Nahezu parallel zu den Ergebnissen der französischen Forscher veröffentlichte Tarchanoff 1890 seine Beobachtungen von Galvanischen Erscheinungen der Haut. Ausgelöst durch äußere Reize, wie beispielsweise durch das Berühren der Haut mit einem Pinsel, bei der Imagination bestimmter Gefühle oder bei geistiger Tätigkeit wie dem Lösen von Rechenaufgaben, beobachtete Tarchanoff eine Veränderung des Stromflusses auf der Haut, die er mit der Absonderung von Schweiß durch eine erhöhte Aktivität der Schweißdrüsen begründete (Tarchanoff 1890). Bereits in diesen grundlegenden Untersuchungen konnte zudem festgestellt werden, dass zum einen ein stärkerer Reiz eine stärkere Veränderung des Hautwiderstandes zur Folge hat, und zum anderen, dass eine Reizfolge zu einer Gewöhnung und damit Abschwächung der Reizantwort führt (Dawson et al. 2000).

Während Vigouroux die Ursache für die Veränderung des Hautwiderstandes in einer veränderten Durchblutung der Haut sah, konnte Darrow 1927 die Überlegungen von Tarchanoff bezüglich der Aktivierung der Schweißdrüsen untermauern. Darrow erfasste 
bei seinen Untersuchungen parallel die Veränderung des Hautwiderstandes und die Schweißsekretion. Er stellte dabei fest, dass die phasische Veränderung des Hautwiderstandes bereits eine Sekunde vor der Registrierung von Schweiß auf der Haut messbar war. Die Folgerung aus diesen Ergebnissen war, dass vor allem die Aktivität der Schweißdrüsen, und nicht der Schweiß auf der Haut selbst, entscheidend für die Veränderung des Hautwiderstandes ist (Darrow 1927).

Die physiologischen Abläufe, die zur Schweißsekretion führen, werden als Sudomotorik bezeichnet. Die Innervation der Schweißdrüsen erfolgt durch sympathische C-Fasern (Macefield et al. 2002). Eine Besonderheit ist, dass bei dieser rein sympathischen Innervation Acetylcholin und nicht Noradrenalin als Transmitter auch postganglionär fungiert. Acetylcholin bindet an muskarinerge Rezeptoren der Schweißdrüse und initiiert so die Schweißsekretion (Sato K und Sato F 1983). Bei der Schweißsekretion werden thermoregulatorisches und emotionales Schwitzen unterschieden. Thermoregulatorisches Schwitzen dient der Abkühlung des Körpers bei körperlicher Anstrengung, während emotionales Schwitzen unter anderem durch psychischen Stress ausgelöst wird (Simon 2000). Die zentrale Regulation des Wärmehaushaltes und damit die Steuerung des thermoregulatorischen Schwitzens findet im Hypothalamus statt (Simon 2000). Signale von peripheren Temperaturfühlern werden über $\mathrm{A} \delta$ - und $\mathrm{C}$-Fasern zum Hypothalamus geleitet (Schlereth et al. 2009). Im Hypothalamus selbst wird zudem die innere Körpertemperatur gemessen (Simon 2000). Ist eine Kühlung der Körperoberfläche zur Senkung der Temperatur erforderlich, so kommt es zur sympathisch vermittelten Schweißsekretion, die beim thermoregulatorischen Schwitzen mit einer Dilatation der peripheren Gefäße einhergeht. Eine Erweiterung der Gefäße, trotz gesteigerter Sympathikusaktivität wird vermutlich durch vasodilatatorisch wirkende Stoffe wie Bradykinin ausgelöst, die von den aktivierten Schweißdrüsen selbst freigesetzt werden (Simon 2000). Während beim thermoregulatorischen Schwitzen vor allem Schweißdrüsen der behaarten Haut aktiviert werden, aktiviert emotionales Schwitzen vorwiegend Schweißdrüsen im Gesicht, im Bereich der Achseln, sowie der plantaren und palmaren Flächen von Füßen und Händen (Schlereth et al. 2009). Im Gegensatz zur Vasodilatation beim thermoregulatorischen Schwitzen geht das emotionale Schwitzen mit einer Vasokonstriktion einher (Simon 2000). Wesentlichen Einfluss auf diese Form des Schwitzens haben sowohl Neokortex als auch das limbische System (Ogawa 1975). Neben den Vitalwerten wie beispielsweise Blutdruck, Herzfrequenz und Tränenfluss ist das Schwitzen ein wichtiger Parameter zur Beurteilung der Narkosetiefe (Schmidt et al. 
2008). Wird nun die elektrodermale Aktivität registriert, so werden ein tonischer und ein phasischer Anteil unterschieden. Als tonisch wird der Basiswert des Hautwiderstandes (Hautwiderstandsniveau, SRL) respektive der Hautleitfähigkeit (Hautleitfähigkeitsniveau, SCL) in Abwesenheit von äußeren Reizeinflüssen bezeichnet, der über einen längeren Zeitraum gemessen wird. Der phasische Anteil stellt die durch einen Reiz ausgelöste Veränderung der elekrodermalen Aktivität dar. Er wird als Hautleitwertsreaktion (SCR) oder Hautwiderstandsreaktion (SRR) bezeichnet (Dawson et al. 2000). Während eine Änderung des tonischen Widerstandes einiger Minuten bedarf, liegt die Änderung des phasischen Anteils im Sekundenbereich. Die Messung der elektrodermalen Aktivität erfolgt als endosomatische oder exosomatischen Messung. Eine endosomoatische Messung registriert die Veränderungen der Leitfähigkeit der Haut direkt, ohne dass von außen Energie hinzu gegeben wird. Exosomatische Messungen hingegen benötigen eine externe Energiequelle, Gleichstrom oder Wechselstrom (Dawson et al. 2000). Die exosomatischen Methoden werden ihrerseits in Konstantstrommessungen und Konstantspannungsmessungen unterteilt. Wie in der jeweiligen Bezeichnung bereits klar wird, ist entweder die applizierte Spannung oder der applizierte Strom während der Messung konstant. Die Konstantspannungsmessungen ermitteln den Hautleitwert G [ $\mu$ siemens], die Konstantstrommessungen den Hautwiderstand R [kOhm]. Da aus dem Widerstand der Leitwert berechnet werden kann und umgekehrt, werden beide Messverfahren außer im Grenzbereich von Spannung oder Strom als gleichwertig angesehen (Winterhalter 2007).

Die in dieser Studie verwendete Messung mittels des Elektrosympathikographen (ESG 1001 Monitor-System, Ingenieurbüro Dr. Janitzki, Altenbeken, Germany) zählt zu den exosomatischen Messungen und wird dort der Gruppe der Konstantstrommessungen zugeordnet (Winterhalter 2007). Lidberg und Wallin konnten mittels elektrischer Reizung von präparierten sympathischen Nervenfasern eine direkte Relation zwischen der Entladungsrate der Nervenfasern und den registrierten Widerstandsänderungen der Konstantstrommessung feststellen (Lidberg und Wallin 1981). Während die elektrodermale Aktivitätsmessung vor allem in der Psychologie und der Physiologie eine große Bedeutung hat, beschränkte sich die Anwendung in der Medizin zunächst auf regionale Anästhesieverfahren (Burk 2005, Zaps 2010). Hier konnten Janitzki und Götte anhand von zeitgleichen Messungen an der oberen und der unteren Extremität die Veränderungen des Hautwiderstandes während einer Spinalanästhesie aufzeigen (Janitzki 
und Götte 1995). Während der Hautwiderstand an der unteren Extremität mit Ausbreitung der Anästhesie zunahm, blieb der Widerstand an der Hand zunächst konstant. Erst bei sehr hohen Spinalanästhesien kam es auch an der oberen Extremität zu einer Zunahme des Hautwiderstandes. Janitzki und Götte erbrachten hierbei den Nachweis, dass die Hautwiderstandsmessung als früher Indikator für eine $\mathrm{zu}$ hohe Ausbreitung einer Spinalanästhesie genutzt werden kann, und somit frühzeitig vor den möglichen kardiovaskulären und pulmonalen Folgen für den Patienten warnen kann (Janitzki und Götte 1995). Entscheidende Untersuchungen zum Verhalten des Hautwiderstandes unter Vollnarkose erfolgten 2002 durch Storm et al. Sie wiesen nach, dass die registrierten Änderungen der Hautleitfähigkeit während einer Narkose direkt mit den Veränderungen des Blutdrucks und der Hormonspiegel von Adrenalin und Noradrenalin korrelierten (Storm et al. 2002). Sie folgerten, dass die Hautleitfähigkeit und die perioperative Sympathikusaktivität bzw. der perioperative Stress in direktem Zusammenhang stehen. Storm konnte in weiteren Studien nachweisen, dass sich die Messung des Hautwiderstandes als objektive, non-invasive Methode zur Messung des individuellen Schmerzempfindens von Patienten eignet (Storm 2008). Aktuelle Studien befassen sich intensiv mit der Möglichkeit, diese Methodik in der Kinderheilkunde anzuwenden, da dort bekanntermaßen große Schwierigkeiten bestehen, um gerade bei Kleinstkindern das Schmerzempfinden $\mathrm{zu}$ evaluieren. Erste Ergebnisse konnten beim Vergleich von etablierten Schmerzskalen und Messungen des Hautwiderstandes einen Zusammenhang zwischen Hautwiderstand und kindlichem Schmerz belegen (Dalal et al. 2012).

Die Validierung des hier verwendeten Elektrosympathikographen zur Messung der Narkosetiefe (ESG 1001 Monitor-System, Ingenieurbüro Dr. Janitzki, Altenbeken, Germany) erfolgte 2007 durch Priv.-Doz. Dr. Michael Winterhalter, der über diese Thematik seine Habilitation „Messung der Hautimpedanz: Ein neuer Weg zur „Narkosetiefebestimmung“ in der Allgemeinanästhesie“ verfasste. In einer ersten Untersuchung konnte durch Winterhalter et al. bei 62 Patienten, die in wachem Zustand unterschiedlichen Reizen ausgesetzt wurden, eine direkte signifikante Korrelation zwischen der Reizintensität, der Abnahme des Hautwiderstandes und der Veränderung der Herzfrequenz nachgewiesen werden. Schmerzreize und akustische Reize führten zu den stärksten Veränderungen der registrierten Parameter (Winterhalter et al. 2007a). Weiterhin wurde die Messung der Sedierungstiefe von Patienten unter Propofol mittels BIS, klinischer Beurteilung der Patienten und ESG-Messung verglichen. Es konnte ein 
signifikanter Zusammenhang der ESG-Messung mit den anderen Parametern und der Propofolplasmakonzentration nachgewiesen werden (Winterhalter 2007). Diese Beobachtungen wurden bei weiteren Untersuchungen, bei denen Narkosen mittels Etomidate und Fentanyl eingeleitet wurden, bestätigt (Winterhalter et al. 2010). Die weiteren Studien von Winterhalter konnten nachweisen, dass bei kardiochirurgischen Operationen unter TIVA die gemessenen Hautwiderstände mit den gleichzeitig bestimmten Vitalparametern und den gemessenen Stresshormonen ADH, Cortisol, ACTH, Adrenalin und Noradrenalin korrelierten (Winterhalter 2007).

\subsubsection{Anatomische und physikalische Eigenschaften der Haut}

Da die Haut eine entscheidende Rolle für die Messung der Sympathikusaktivität spielt, soll hier kurz auf die wesentlichen, für diese Arbeit maßgeblichen, anatomischen und physiologischen Eigenschaften des größten Organs des menschlichen Körpers eingegangen werden. Die Haut bedeckt den gesamten menschlichen Körper. Sie dient dem Schutz vor äußeren Einflüssen, und sie ermöglicht durch ihre große Anzahl an Rezeptoren für Tastsinn, Temperatur und Schmerz die Kommunikation mit der Umwelt. Die gesamte Haut des erwachsenen Menschen misst zwischen 1,2 bis $2,3 \mathrm{~m}^{2}$, bei einer Dicke von 1,5 bis $4 \mathrm{~mm}$. Die Haut besteht aus den beiden Schichten Epidermis und Dermis. Unter der Haut liegt die Subkutis. Haare, Nägel, Talg- und Schweißdrüsen werden als Hautanhangsgebilde bezeichnet (Gratzl 2002). Die Epidermis kann von außen nach innen in fünf verschiedene Schichten von Keratinozyten (Stratum corneum, lucidum, granulosum, spinosum und basale) unterteilt werden. Stratum papillare und reticulare bilden die Dermis. Der Schutz des Körpers ist die Hauptaufgabe der Epidermis, sie verhindert eine Austrocknung sowie das Eindringen von Fremdkörpern. Die bindegewebige Dermis unterstützt die Epidermis und verzahnt sie mit den darunter liegenden Schichten. Sie enthält den sekretorischen Teil der ekkrinen Schweißdrüsen, die zu den Hautanhangsgebilden zählen. Die Schweißdrüsen sind tubuläre Drüsen, deren Ausführungsgänge ohne Verzweigungen die Epidermis durchziehen und auf dem Stratum corneum enden (Gratzl 2002). Insgesamt besitzt der Mensch circa zwei Millionen Schweißdrüsen (Schiebler et al. 1999a). Mit wenigen Ausnahmen - z.B. der Glans penis sind diese Drüsen auf der gesamten Körperoberfläche zu finden (Gratzl 2002). An Handflächen, Fußsohlen und Achseln findet sich die größte Dichte von Schweißdrüsen (Sato K et al. 1989). 
Der Schweiß ist ein Ultrafiltrat des Blutes. Er wird durch die Kontraktion von Myoeptithelzellen aus den mukösen Zellen der Schweißdrüsen, die die Sekretgranula enthalten, freigesetzt. Bei einem pH-Wert von 4,5 besteht der Schweiß im Wesentlichen aus Wasser, Natriumchlorid, Harnstoff, Ammoniak und Harnsäure. Während der Anteil von Natrium im Blut bei 140-160 mmol/l liegt, hat der Schweiß einen Natriumanteil von 20-60 mmol/l (Gratzl 2002). Die im Schweiß enthaltenden Ionen machen einen Transport elektrischer Ladungen möglich. Eine Änderung dieser Ladungsträger, die mit der Aktivität und Funktion der Schweißdrüsen in direktem Zusammenhang steht, beeinflusst somit auch die Leitfähigkeit und den Widerstand der Haut (Zaps 2010). Der Stromfluss durch die Haut findet entweder an der Hautoberfläche entlang der Epidermis statt oder durch Dermis und Subkutis (Chizmadzhev et al. 1998). Den größten Widerstand für den Stromfluss bildet die Epidermis (Becker 2008). Diesen Nachweis erbrachten Yamamoto et al. Die Forscher brachten Klebestreifen auf der Haut an, die beim Abziehen durch die Anhaftung von Zellen die Lipid-Keratozyten-Matrix des Stratum corneum reduzierten. Dieses Verfahren wird als „Tape Stripping“ bezeichnet. Anschließend konnten sie eine deutliche Reduktion des Hautwiderstandes nachweisen (Yamamoto und Yamamoto 1976). Jede Veränderung des Zustandes des Stratum corneum, ob eine Änderung der Hydratation, eine Verletzungen oder eine Abschilferung, kann demnach den Hautwiderstand beeinflussen. Der Widerstand der tiefer gelegenen Schichten Dermis und Subkutis ist deutlich geringer (Kalia und Guy 1995, Zaps 2010).

\subsubsection{Physikalische Grundlagen der Sympathikographie}

Bei der Bestimmung der Sympathikusaktivität ist der Widerstand der Haut gegenüber dem angelegten Strom von entscheidender Bedeutung (s.o.). Die physikalischen Grundlagen dazu sollen im Folgenden erläutert werden. Der elektrische Widerstand R, den geladene Teilchen überbrücken müssen, ist abhängig von der anliegenden Spannung U und der Stromstärke I. Die Einheit des Widerstandes ist Ohm

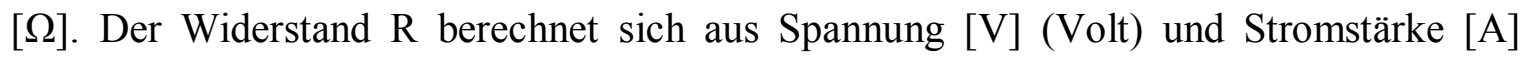
(Ampère) wie folgt: $\mathrm{R}[\Omega]=\mathrm{U}[\mathrm{V}] / \mathrm{I}[\mathrm{A}]$ (Chizmadzhev et al. 1998). Nach dem Ohmschen Gesetz sind Spannung und Stromstärke proportional, damit ist der Widerstand konstant. Der Widerstand ist abhängig von der Länge [1], dem Querschnitt [A], und dem Material des durchflossenen Leiters. Um dies zu berücksichtigen, wird für jedes Material der spezifische elektrische Widerstand $\rho$ (Rho) angegeben, der besagt, wie groß der Widerstand des jeweiligen Materials bei einer Länge [1] von $1 \mathrm{~m}$ und einem 
Querschnitt [A] von $1 \mathrm{~mm}^{2}$ ist. Bei der Berechung eines elektrischen Widerstandes gilt dann $\mathrm{R}=\rho \times 1 / \mathrm{A}$. Durch Umformen lässt sich die Einheit von $\rho$ berechnen: $\left[\Omega \times \mathrm{mm}^{2} / \mathrm{m}\right]$ (Bergmann F et al. 1994, Zaps 2010).

Für die Haut als elektrischer Leiter ist eine Angabe eines einheitlichen spezifischen elektrischen Widerstands nicht möglich. Je nach Ort der Messung differieren die Werte ebenso bei ein und derselben Person (intraindividuell), als auch zwischen verschiedenen Personen (interindividuell) (Becker 2008, Winterhalter et al. 2007b, Zaps 2010). Nach der Berechnung des elektrischen Widerstandes lässt sich aus diesem direkt der jeweilige Leitwert $G[S]$ (Siemens) berechnen: $G=1 / R$.

\subsubsection{Perioperative Sympathikusaktivität und Narkosetiefe}

Im Folgenden wird auf die weiteren Möglichkeiten der perioperativen Messung der Sympathikusaktivität und Bestimmung der Narkosetiefe eingegangen. Dieses Kapitel erhebt keinen Anspruch auf Vollständigkeit, da vor allem die in dieser Studie verwendeten Verfahren und ihr Hintergrund näher beleuchtet werden sollen.

Um jeden Patienten vor einer überschießenden sympathischen Aktivität zu schützen, ist das Erreichen einer ausreichenden Narkosetiefe von entscheidender Bedeutung. Seit den Anfängen der Anästhesie wurde nach Möglichkeiten gesucht, um die Tiefe einer Narkose zu erfassen. Die erste Graduierung der Narkosetiefe stammt aus dem Jahr 1847 und wurde von John Snow entwickelt (Schmidt et al. 2008). Das eingängigste Schema, das für die Zeit der Äthernarkosen Anwendung fand, geht auf Arthur Guedel zurück und stammt aus dem Jahr 1920. Zentrales Element dieses Schemas war die klinische Beobachtung des Patienten. Veränderungen von Atmung, Pupillenstatus, Bewegungen der Augen, die Aktivität der Reflexe des Patienten und Änderungen des Vegetativums dienten zur Einteilung in eines der vier Narkosestadien, Amnesie und Analgesie, Exzitation, chirurgische Toleranz oder Vergiftung (Larsen 2010b). Da in der heutigen Zeit die Schmerztherapie während einer Narkose mit Opiaten durchgeführt wird, und häufig Muskelrelaxantien eingesetzt werden, kann das von Guedel entwickelte Schema nur noch bedingt angewendet werden (Schmidt et al. 2008). 1987 entwickelte John M. Evans den PRST-Score. Das Akronym PRST steht für die Parameter Pressure, Rate, Sweating und Tearing. Evans fasste die klinische Beobachtung von Blutdruck, Herzfrequenz, Schweißsekretion und Tränenfluss in einer Punkteskala zusammen. Die Summe der einzelnen Parameter liegt zwischen null und acht. Ein Wert, der größer ist als zwei, weist auf eine zu flache Narkose hin (Evans 1987). Mittlerweile ist bekannt, dass alle im PRST- 
Score berücksichtigten Parameter einer großen individuellen Schwankungsbreite unterliegen. Die Aussagekraft des PRST-Scores ist deutlich eingeschränkt. So wurden in den USA Fälle von intraoperativer Wachheit aus den Jahren 1960 bis 1995 untersucht. Nur bei $15 \%$ der Patienten fand sich bei der Untersuchung der Narkoseprotokolle ein Blutdruckanstieg, bei 7\% eine Zunahme der Herzfrequenz und lediglich bei $2 \%$ der Patienten wurde eine intraoperative Bewegung registriert (Domino et al. 1999). Ferner werden beispielsweise die Herzfrequenz und der Blutdruck sowohl durch die Vormedikation des Patienten als auch durch die intraoperativ angewendeten kreislaufstabilisierenden Medikamente maßgeblich beeinflusst.

\subsubsection{EEG, BIS, evozierte Potentiale}

Mit der Entdeckung der elektrischen Aktivität der Nervenzellen, die auf der Oberfläche des Gehirns erfasst werden kann, ergab sich eine weitere Möglichkeit, die Narkosetiefe zu bestimmen. Die elektrischen Signale, die auf der Hirnoberfläche messbar sind, werden durch Pyramidenzellen erzeugt, die vertikal zur Hirnoberfläche stehen. Erregte Hirnareale sind durch eine negative, nicht erregte Areale durch eine positive Ladung gekennzeichnet. Gemeinsam bilden negative und positive Areale einen Dipol. Die Summe der Dipole erzeugt Potentiale, die auf der Kopfhaut als Elektroenzephalogramm (EEG) gemessen werden können. Da die Aktivität der oberflächlich gelegenen Pyramidenzellen maßgeblich durch eine Vielzahl von Verbindungen zu subkortikalen Arealen beeinflusst wird, ist das EEG ein Abbild der gesamten Hirnaktivität (Schmidt et al. 2008). Die ersten EEG Untersuchungen am Menschen wurden durch Hans Berger 1923 durchgeführt. Er untersuchte die EEG-Veränderungen während Chloroformnarkosen (Berger 1929). Die Ableitung des EEG erfolgt durch Elektroden, die auf der Kopfhaut angebracht werden. Der Frequenzbereich reicht von $0-80 \mathrm{~Hz}$ und die Amplituden von 1-100 $\mu \mathrm{V}$ (Birbaumer und Schmidt 2000). Von entscheidender Bedeutung bei der Interpretation des EEG sind die Frequenz der Schwingungen und die Morphologie der aufgezeichneten EEG-Wellen (Schmidt et al. 2008). Unterschieden werden $\alpha-, \beta-, \gamma-, \zeta-$ und $\delta$-Wellen. Die Frequenz der $\alpha$-Wellen liegt in einem Bereich von 8-13 Hz. In wachem Zustand mit geschlossenen Augen finden sich im EEG vor allem $\alpha$-Wellen. Der Patient ist wach, konzentriert sich aber nicht auf etwas Bestimmtes. $\alpha$-Wellen treten meistens synchronisiert auf. Bei einem synchronisierten EEG werden an einem Messpunkt überwiegend Wellen mit gleicher Frequenz und Amplitude aufgezeichnet. Wirken auf den Patienten Sinnesreize ein, so werden die $\alpha$-Wellen von $\beta$-Wellen mit einer 
Frequenz von 15-30 Hz abgelöst, diese sind meist desynchronisert. Bei erhöhter geistiger Arbeit treten $\gamma$-Wellen auf, die mit einer Frequenz über $30 \mathrm{~Hz}$ imponieren. Niederfrequente Wellen mit großer Amplitude sind die $\zeta$ - und $\delta$-Wellen, die das SchlafEEG kennzeichnen. $\zeta$-Wellen liegen im Frequenzbereich von 4-7 Hz, $\delta$-Wellen im Bereich von 0,1-4 Hz. Nur im Kindesalter und bei krankhaften Veränderungen finden sich diese Wellen auch im Wachzustand (Birbaumer und Schmidt 2000). Die Medikamente, die während einer Narkose eingesetzt werden, bedingen eine Änderung des EEG von wachen zu schlafähnlichen Frequenzen und Amplituden. Da die Interpretation der Rohdaten des EEG äußerst kompliziert ist und eine unmittelbare intraoperative Interpretation allein aufgrund der großen Datenmenge - die Aufzeichnung erfolgt in der Regel mit $30 \mathrm{~mm} / \mathrm{s}$ - nahezu unmöglich ist, stehen verschiedene Verfahren zur rechnerischen Interpretation zur Verfügung. Um das EEG zeitnah verarbeiten zu können wird eine Spektralanalyse durchgeführt, bei der kurze EEG-Abschnitte von beispielsweise 5 oder 10 Sekunden prozessiert werden. Diese Analyse wird als Fast-FourierTransformation (FFT) bezeichnet. Ihm liegt die physikalische Annahme zugrunde, dass die aufgezeichneten EEG-Wellen aus Sinus- und Kosinusschwingungen zusammengesetzt sind. Die untersuchten EEG-Abschnitte werden auf Korrelationen mit vorliegenden EEGs hin untersucht. Das Ergebnis des FFT wird als Leistungs- oder Powerspektrum bezeichnet und ermöglicht eine Darstellung der vorherrschenden Frequenzbereiche des zu untersuchenden EEG-Bereichs. Durch die Berechnung der Fläche des Leistungsspektrums für die einzelnen Wellenbereiche ist eine noch genauere Analyse des FFT-Ergebnisses möglich. Die einzelnen Frequenzbänder können als absolute Zahl [uV²] oder als prozentualer Anteil an der Gesamtaktivität [\%] dargestellt werden. Aus der Analyse des Leistungsspektrums lässt sich ferner die Medianfrequenz, als Frequenz unter- und oberhalb der 50\% der EEG-Aktivität liegen, errechnen. Bei der Durchführung chirurgischer Eingriffe wird eine Medianfrequenz im Bereich von 2-3 Hz empfohlen. Weiterhin ist die spektrale Eckfrequenz (SEF) ersehbar. Mit der spektralen Eckfrequenz (SEF) wird die Frequenz des EEG angegeben, unter der 95\% (SEF 95) oder 90\% (SEF 90) der Gesamtaktivität des EEG liegen. Die SEF für operative Eingriffe sollte im Bereich von 8-12 Hz liegen (Schmidt et al. 2008). Trotz seiner Empfindlichkeit ist das EEG nicht uneingeschränkt zur Beurteilung der Narkosetiefe geeignet. Bruhn konnte die Limitierung des EEG und der daraus berechneten Parameter zur Beurteilung der Narkosetiefe zeigen. Seine Untersuchungen ergaben, dass bei einer geringen Anästhetikakonzentration während der Narkoseeinleitung die hohen EEG-Frequenzanteile zunehmen, die SEF 95 
steigt folglich an. Ferner konnte eine zunehmende EEG-Aktivität trotz tiefer Narkose beobachten werden (Bruhn 2003). Der paradoxe Wechsel zwischen hoch- und niederfrequenten EEG-Frequenzen wird als „Burst-suppression“ - Muster bezeichnet. Das Auftreten von Bursts trotz hoher Anästhetikakonzentration lässt eine alleinige Beurteilung der Narkosetiefe mittels EEG nicht zu (Bruhn 2003). Ferner können sowohl Veränderungen der Hirnfunktion als auch die Einflussnahme von Außen durch unterschiedliche Pharmaka zu einer Veränderung der Hirnaktivität und damit des erstellten EEGs führen. Die Modulation des EEG, die beispielsweise durch eine Hypoglykämie oder eine verminderte zerebrale Durchblutung verursacht wird, ist der einer Narkose sehr ähnlich. Medikamente wie Ketamin oder Lachgas, die in der Narkoseführung häufig noch Anwendung finden, können ebenfalls modulierend auf das EEG Einfluss nehmen. Die Verwendung von Ketamin bedingt eine Veränderung der $\beta-, \zeta-$ und $\delta$-Wellen, was die Verwertung des EEG deutlich einschränkt, da es zu widersprüchlichen EEG-Veränderungen kommt. Wohingegen Lachgas eine Narkose des Patienten auslösen kann, ohne dass es zu entsprechenden Veränderungen im EEG kommt. Dieser Effekt ist bedingt durch die Tatsache, dass Lachgas vor allem im Hirnstamm und Rückenmark, und nicht im Kortex wirkt (Schmidt et al. 2008).

Um die immensen Informationen des EEG leichter zugänglich zu machen und seine Aussagekraft bei der Messung der Narkosetiefe zu verbessern wurde das BIS-Monitoring (Bispectral-Index) entwickelt. Der BIS wird als dimensionslose Zahl zwischen 0 und 100 dargestellt. Der Name impliziert bereits die zugrunde liegende mathematische Funktion, die Bispektralanalyse. Mit der Bispektralanalyse wird hier die Biokohärenz, also das Verhältnis der zugrunde liegenden Sinusschwingungen des EEG beschrieben (Lehmann et al. 2001). Der genaue Algorithmus, der der BIS-Berechnung zugrunde liegt, wurde jedoch durch die Entwickler bisher nicht veröffentlicht. Bekannt ist aber, dass in der Bispektralanalyse Parameter aus der Analyse des konventionellen EEGs wie FFT (FastFourier-Transformation), Spektralanalyse und Burst-Supression-Verhältnis gemeinsam mit Bispektralparametern verwendet werden (Rampil 1998). Bei der Entwicklung des BIS wurde zudem auf eine große Sammlung von EEGs mit den entsprechend dokumentierten Verhaltensmustern der Patienten zurückgegriffen. Die mathematische Analyse der Daten hatte das Ziel, eine Bewegung des Patienten auf einen Schmerzreiz vorherzusagen (Lehmann et al. 2001). Ergebnis dieser komplexen Berechnung ist ein BIS zwischen 0 und 100, dessen Erläuterung in Tabelle 1 dargestellt ist. 


\begin{tabular}{l|l}
\hline & Narkosetiefe, Bewusstseinsstatus \\
\hline \hline BIS & Wach, Erinnerungsvermögen intakt \\
$100-90$ & Sedierung \\
$85-65$ & ausreichende Narkosetiefe bei Allgemeinanästhesie \\
$40-60$ & tiefe Narkose, fehlendes unbewusstes Erinnerungsvermögen \\
$40-35$ & beginnende burst-suppression \\
$<40$ & zunehmende burst-suppression \\
$<30$ & Nulllinien-EEG \\
0 &
\end{tabular}

Tabelle 1: Erläuterung der BIS-Werte (ab Version 3.0) (Lehmann et al. 2001)

Das BIS wurde in vielen Studien untersucht, und die Korrelation zu den Narkosestadien einer Sedierung mit Midazolam oder Propofol konnte nachgewiesen werden (Glass et al. 1997, Liu et al. 1996, Liu et al. 1997). Ebenso konnte bei der Kombination von Propofol mit dem Opioid Alfentanil und bei den volatilen Anästhetika Isofluran und Sevofluran eine gute Korrelation des BIS mit dem erreichten Sedierungsgrad beobachtet werden (Glass et al. 1997, Iselin-Chaves et al. 1998, Katoh et al. 1998). Auch aus ökonomischer Sicht zeigten sich bei der Verwendung von BIS deutliche Vorteile. Studien belegten eine signifikante Reduktion des Narkosemittelbedarfs sowie eine schnellere Verlegung aus dem Aufwachraum bei Narkosen, die mittels BIS gesteuert wurden (Añez et al. 2001, Song et al. 1997). Obwohl die Vorteile und die Wirksamkeit von BIS vielfach belegt wurden, so konnten in weiteren Studien auch Einschränkungen festgestellt werden. Beispielsweise ist die Steuerung einer Ketanestnarkose mit BIS nicht möglich, ferner führte die Kombination einer Propofolnarkose mit Ketanest zu einer tieferen Sedierung, ohne dass sich durch die additive Gabe von Ketanest eine Änderung der vorbestehenden BIS-Werte ergab. Weiterhin wurde festgestellt, dass BIS nicht dazu verwendet werden kann, um die auftretenden pharmakologischen Effekte wie Schmerzlinderung und Sedierungssteigerung, die sich bei der Hinzunahme von Lachgas zeigen, zu überwachen (Lehmann et al. 2001).

In einer aktuellen Studie von Avidan et al. aus 2011 wurde bei 6041 Patienten die Narkosetiefe während einer Vollnarkose entweder mit BIS oder mit ETAC (end-tidal anesthetic-agent concentration) überwacht. Die Forscher stellten in der BIS-Gruppe einen höheren Anteil von Awareness fest (Avidan et al. 2011). Zusammenfassend kann man zum jetzigen Zeitpunkt sagen, dass mit BIS ein wichtiger zusätzlicher Parameter zur 
Narkoseüberwachung zur Verfügung steht, der aber letztlich noch nicht zur alleinigen Überwachung der Narkosetiefe ausreicht.

Eine weitere spezielle Form des EEG, auf die hier kurz eingegangen werden soll, stellen die akustisch-evozierten (AEP) und die somatosensorisch-evozierten Potentiale (SEP) dar. Bei den AEP werden nach Abgabe eines akustischen Reizes die entsprechenden Veränderungen der Hirnströme, die eine Reizantwort darstellen, von der Kopfhaut, ähnlich dem EEG, abgeleitet. Diese Signale müssen aus dem EEG herausgefiltert und anschließend gemittelt werden, da sie eine um ein Vielfaches kleinere Amplitude aufweisen. Zur Beurteilung der AEPs wird eine Abfolge von Reizantworten gemittelt. AEPs können nur alle 1-3 min erfasst werden, trotz eines Reizes erfolgt in der Zwischenzeit keine Antwort. Neue AEPs fließen direkt in die Mittelung ein und das dann älteste AEP wir aus der Mittelung gestrichen. Das ,aktuell“ angezeigte AEP beruht also unter anderem auf Messungen aus der Vergangenheit (Schmidt et al. 2008). AEPs werden anhand ihres Auftretens nach einem Reiz in frühe $(0-10 \mathrm{~ms})$, mittel-latente (MLAEP) (10-100 ms) und späte (100-1000 ms) AEPs unterteilt (Winterhalter 2007). Es konnte gezeigt werden, dass die AEPs geeignet sind, um die Narkosetiefe zu bestimmen. Analgetika wie Ketanest und Fentanyl oder Benzodiazepine zeigten keinen Einfluss auf die AEPs, volatile Anästhetika führten abhängig von ihrer Dosis zwar zu einer Abschwächung der Messungen, was jedoch im Rahmen einer Awareness durch die zunehmende Hirnaktivität ausgeglichen wurde. Eine Aussage über eine ausreichende Analgesie ist mittels AEP nicht möglich (Freye et al. 2000). Somatisch-evozierte Potenziale scheinen hingegen für das Monitoring einer ausreichenden Analgesie geeignet zu sein. Zur Auslösung eines somatisch-evozierten Potenzials wird fünf Mal pro Sekunde ein Reiz von 0,5 ms Dauer mit einer Stärke, die $1 \mathrm{~mA}$ über der Grenze zur Auslösung einer motorischen Antwort liegt, am Nervus medianus appliziert. Die Auswertung entspricht dann im Wesentlichen der der akustisch evozierten Potenziale. Die Gabe von Analgetika führt, je nach Potenz des Analgetikums zu einer erneuten Reduktion der Amplitude (Freye et al. 2000). Mast et al. konnten zeigen, dass SEPs des Nervus medianus Anästhetikaeffekte nachweisen und zur Beurteilung der Narkosetiefe beitragen können (Mast 2009).

\subsubsection{Surgical Pleth Index}

Der Surgical Pleth Index (SPI) oder auch Surgical Stress Index (SSI) stellt eine neuere Methode zur Beurteilung der perioperativen Sympathikusaktivität und Nozizeption 
dar, die zurzeit in vielen Studien intensiv untersucht wird. Grundlage des SPI ist die periphere Vasokonstriktion, die maßgeblich durch die zentrale sympathische Aktivität induziert wird. Photoplethysmographische Messungen sollen Rückschlüsse auf die zentrale sympathische Aktivität ermöglichen (Huiku et al. 2007). Diese Methode wurde von Huiku et al. entwickelt, die plethysmographische und elektrokardiographische Untersuchungen bei Patienten unter Allgemeinanästhesie durchführten. Sie kombinierten die Messung der normalisierten Pulswellenamplitude (PPGA ${ }_{\text {norm}}$ ) und des normalisierten Herzschlagintervalls $\left(\mathrm{HBI}_{\text {norm}}\right)$ und berechneten daraus anhand folgender Formel den Surgical Stress Index: SSI $=100-\left(0,7 * \mathrm{PPGA}_{\text {norm }}+0,3 * \mathrm{HBI}_{\text {norm }}\right)$. Huiku et al. konnten bei ihrer Untersuchung feststellen, dass der SSI auf perioperative Schmerzreize und auf Veränderungen der applizierten Remifentanildosis reagierte (Huiku et al. 2007). Der SPI wird als dimensionsloser Index von 0-100 dargestellt, wobei 0 auf eine geringe und 100 auf eine sehr hohe sympathische Aktivität hinweisen soll (Ludwigs 2011). In mehreren Studien unter Allgemeinanästhesie konnte bereits nachgewiesen werden, dass der SPI geeignet ist, um intraoperative Schmerzreize darzustellen. So führten nozizeptive Reize eher zu einer Veränderung des SPI als zu einer Veränderung der Messung von Blutdruck oder Herzfrequenz (Chen et al. 2012). Struys et al. konnten ferner eine gute Korrelation des SPI mit der Remifentanilkonzentration bei intraoperativen Schmerzreizen nachweisen. Sie folgerten, dass mittels des SPI eine gute Steuerung der perioperativen analgetischen Therapie des Patienten möglich ist (Struys et al. 2007). Chen et al. konnten beim Vergleich von Allgemeinanästhesien, bei denen die Analgetikagabe in Abhängigkeit vom SSI erfolgte, und Allgemeinanästhesien ohne SSI, einen geringeren Remifentanilbedarf bei besserer hämodynamischer Stabilität unter SSI-Monitoring nachweisen (Chen et al. 2010). Ähnliche Ergebnisse zeigten auch Untersuchungen von Bergmann I et al., bei denen unter SSI-Monitoring geringere Dosierungen von Remifentanil und Propofol bei Allgemeinanästhesien benötigt wurden, und die postoperative Erholung signifikant schneller erfolgte (Bergmann I et al. 2012). Trotz der insgesamt viel versprechenden Ergebnisse entdeckten einige Studien gerade bei wachen Patienten auch Einschränkungen des SPI. So zeigten die Untersuchungen von Ludwigs, dass die äußeren Reize, die bei wachen Patienten unter Spinalanästhesie auf den Patienten einwirken, eine Veränderung des SPI trotz Schmerzfreiheit verursachen. Ferner konnte bei diesen Untersuchungen ein Einfluss der Lagerung des Patienten auf die SPI-Werte nachgewiesen werden (Ludwigs 2011). Insgesamt aber deuten die bisherigen Ergebnisse daraufhin, dass der Surgical Pleth 
Index eine viel versprechende Methode zum perioperativen Monitoring der Sympathikusaktivität zu sein scheint. 


\section{Fragestellung}

In der modernen Anästhesie gibt es immer wieder große Herausforderungen, weil man es regelmäßig mit Patienten in Extremsituationen im Operationssaal zu tun hat. Die Patienten wissen durch Aufklärung und Selbststudium, dass eine Anästhesie ebenso wie die jeweilige Operation Risiken beinhaltet, dies verursacht Ängste und Stress. Die anästhesiologische Behandlung mit den unterschiedlichen Verfahren der Allgemein- und der Regionalanästhesie ist in den letzten Jahren wesentlich verbessert worden. Das Interesse von Anästhesisten besteht darin, für jeden Patienten das individuell bestmögliche Anästhesieverfahren durchzuführen. Das perioperative anästhesiologische Risiko der Patienten resultiert aus Komplikationen von Vorerkrankungen, psychischen Belastungen und komplikationsträchtigen anästhesiologischen Maßnahmen. Für optimale Behandlungsergebnisse ist neben der Qualifikation und Ausbildung der behandelnden Anästhesisten, die richtige Auswahl des Anästhesieverfahrens und die Optimierung des Monitorings von Bedeutung. Monitoring bedeutet kontinuierliche bzw. nahezu kontinuierliche Visualisierung von physiologischen Funktionen. Es sollte gezielt eingesetzt werden, damit gefährliche Störungen von Vitalfunktionen der Patienten minimiert werden und pathologische Reaktionsmechanismen des Körpers frühzeitig erfasst werden. Der Funktionszustand des zentralen und vegetativen Nervensystems nimmt in der Anästhesie eine wichtige Rolle als Zielorgan des anästhesiologischen Handelns ein. Ein Blick in all diese Schaltzentralen bei perioperativen Belastungen erfordert ein hohes Maß an Verständnis für die Steuer- und Regelmechanismen und deren Auswirkungen auf die Organsysteme. Die heute zur Verfügung stehenden respiratorischen und kardiozirkulatorischen Monitoringsysteme erlauben eine umfassende Überwachung der entsprechenden Funktionen. Dieses Monitoring scheint für die Routine der klinischen Anästhesie ausreichend.

Im Bereich der kortikalen und neurovegetativen Aktivität stellen sich die Verhältnisse intraoperativ anders dar. Zwar wird das EEG zunehmend für die Überwachung von kortikalen Funktionen eingesetzt, dieser Parameter stellt jedoch nur einen Teilaspekt der für die Anästhesie bedeutsamen Abläufe im ZNS und Neurovegetativum dar (Wilhelm und Kreuer 2003). Das EEG erlaubt Rückschlüsse auf den Hypnosegrad eines anästhesierten Patienten, nicht aber auf den Funktionszustand vegetativer Zentren und auf das Ausmaß induzierter und spontaner sympathischer Reaktionen (Wallenborn 2012). 
Seine Aussagekraft ist gerade in neueren Untersuchungen in Frage gestellt worden (Avidan et al. 2011).

Der Schutz des Patienten vor zu hoher, unphysiologischer sympathischer Aktivität, die negative Folgen für den weiteren Krankheitsverlauf des Patienten haben kann, gehört zu den besonders wichtigen Zielen in der Anästhesie. Tierexperimentell und in klinischen Studien konnte nachgewiesen werden, dass eine überschießende Sympathikusaktivierung einen negativen Einfluss auf die Immunabwehr des Körpers und damit auf den postoperativen Verlauf hat (Ben-Eliyahu et al. 1999, Herwig et al. 2003). Ein Stressmonitoring mit Erfassung der Sympathikusaktivität als mögliche Ergänzung des EEGs erscheint somit zur Erfassung der zu erwartenden Belastungen der Patienten wünschenswert. Mit der Sympathikographie steht ein Verfahren zur Verfügung, das indirekt, durch Messung des Hautwiderstandes, die Sympathikusaktivität und damit den perioperativen Stress messen kann (Storm et al. 2002).

Es stehen heute verschiedenste anästhesiologische Möglichkeiten zur Verfügung um einer perioperativ zu stark erhöhten Sympathikusaktivität vorzubeugen bzw. um auf diese Einfluss zu nehmen. Bei Operationen an der Hand bietet sich beispielsweise die axilläre Plexusblockade als eine Form der Regionalanästhesie an. Doch obwohl der Patient nach erfolgreicher Plexusblockade keine chirurgischen Schmerzreize mehr empfindet, ist er während der Operation schon allein durch die ungewohnte Umgebung im OP-Saal Stress ausgesetzt. Noch ist weitestgehend unbekannt, inwiefern, trotz ausreichender Analgesie, unbewusste Stressreaktionen beim wachen Patienten unter Regionalanästhesie vorhanden sind und welche Auswirkungen sie haben.

Im Fokus dieser Studie steht die Beobachtung der perioperativen sympathischen Aktivität bei handchirurgischen Operationen durch Messung der indirekten Parameter Sympathikographie, Herzfrequenz, mittlerer arterieller Druck und Hauttemperatur. Folgende Fragestellungen stehen dabei im Blickpunkt: Lässt sich anhand der erfassten Parameter ein Unterschied in der Sympathikusaktivität der Patienten unter axillärer Plexusanästhesie oder Allgemeinanästhesie nachweisen? Kann eine Regionalanästhesie die perioperative Reaktion des Sympathikus effektiver dämpfen als eine Allgemeinanästhesie? Gibt es neben dem chirurgischen Trauma weitere Faktoren, die im Hinblick auf die Stressreaktion des Patienten eine Rolle spielen? 


\section{Methoden}

Zwei Gruppen á 23 Patienten, die sich in ultraschallgesteuerter axillärer Plexusblockade (axPlex) oder Allgemeinanästhesie (ITN) einer Operation unterziehen mussten, wurden nach Genehmigung durch die Ethikkommission untersucht. Die Symphatikusaktivität wurde anhand des Hautwiderstands (ESG 1001, Ingenieurbüro Dr. Janitzki, Altenbeken, Germany) gemessen, die Herzfrequenz und der mittlere arterielle Druck als indirekte Sympathikusindikatoren erfasst. Ferner wurde die Hauttemperatur an der nicht zu operierenden Hand gemessen. Die Narkosetiefe der ITN wurde mit BIS überwacht. Das peri- und postoperative Schmerzempfinden wurde mittels einer numerischen Ratingskala erfasst. Die Messung umfasste insgesamt sieben Messzeitpunkte $\left(\mathrm{T}_{\text {Ausgangswert }}, \mathrm{T}_{\text {Narkosebeginn }}, \mathrm{T}_{\text {Blutsperre }}, \mathrm{T}_{\text {Schnitt }}, \mathrm{T}_{\text {OP-Ende }}, \mathrm{T}_{\text {Aufwachraum }}\right.$, $\left.\mathrm{T}_{\text {Normalstation }}\right)$.

\subsection{Patienten}

46 Patienten nahmen an der Studie teil, die mit einer Genehmigung der Ethikkommission der Universität Göttingen (Antragsnummer 7/10/10) durchgeführt wurde. Alle Patienten unterzogen sich Operationen in der handchirurgischen Abteilung des St. Vincenz Krankenhauses in Paderborn. Bei diesen handchirurgischen Eingriffen handelte es sich überwiegend um Karpaldachspaltungen, Ringbandspaltungen und Osteosynthesen, die als ambulante Operation je nach Studiengruppe in Plexusanästhesie (axPlex) oder Allgemeinanästhesie (ITN) durchgeführt wurden. Ausschlusskriterien waren ein Alter $<18$ Jahre, um die volle Geschäftsfähigkeit der Patienten zu gewährleisten, oder ein Alter $>80$ Jahre, da bei diesen Patienten eine ambulante Versorgung meist nicht möglich ist. Weiterhin wurden Patienten mit einer ASAKlassifikation > III, sowie Patienten mit einer Vorerkrankung, die eine Kontraindikation gegen die verwendeten Medikamente oder Narkosenformen darstellte, ausgeschlossen. Nach ausführlicher mündlicher und schriftlicher Aufklärung über die beiden möglichen Narkoseverfahren (axilläre Plexusanästhesie vs. Allgemeinanästhesie) und anschließender Einwilligung der Patienten konnten diese die Narkoseform frei wählen. Die Einteilung der Patienten in die ITN- oder die axPlex-Gruppe erfolgte anhand der Entscheidung des Patienten. 
Am Tag der Operation erhielten alle Patienten eine Stunde vor der Operation eine orale Prämedikation mit Dikalium-Clorazepat (Tranxilium®, Sanofi-Aventis Deutschland $\mathrm{GmbH}$, Frankfurt am Main, Deutschland) $20 \mathrm{mg}$ und Ranitidin (Ranitic $®$, Hexal, Holzkirchen, Deutschland) $150 \mathrm{mg}$. Anschließend wurden sie in den Aufwachraum gebracht.

Im Aufwachraum wurden die Patienten mit einem peripheren venösen Zugang versorgt und an das Basismonitoring bestehend aus Blutdruckmessung, Herzfrequenzüberwachung und Messung der Sauerstoffsättigung angeschlossen. Das ESG wurde an Daumen und Zeigefinger der nicht zu operierenden Hand, an der auch die Temperatur gemessen wurde, angebracht. Zur anonymen Datenauswertung erhielt jeder Patienten eine fortlaufende Nummer. Die erhobenen Daten bestehend aus Herzfrequenz [1/min], Blutdruck [mmHg], Sauerstoffsättigung [\%], Hautwiderstand $[\mathrm{kOhm}]$ und Hauttemperatur $\left[{ }^{\circ} \mathrm{C}\right]$ wurden im Abstand von fünf Minuten sowie bei besonderen Ereignissen notiert. In der Gruppe der Allgemeinanästhesien wurden zusätzlich die BIS-Werte, der expiratorische $\mathrm{CO}_{2}$-Partialdruck [mmHg], die expiratorische Sevofluran (Sevorane®, Abbot GmbH \& Co. KG, Wiesbaden, Deutschland)-Konzentration und der MAC-Wert des Narkosegases erfasst.

\subsubsection{Handchirurgie}

In diesem Kapitel soll kurz auf die studienrelevanten, handchirurgischen Grundlagen eingegangen werden. Karpaltunnelsyndrome (KTS), Ringbandstenosen (RBS) und kleinere Frakturen bildeten den Großteil der operationsbedürftigen Krankheitsbilder, die bei den Studienpatienten vorlagen.

Beim Karpaltunnelsyndrom (KTS) handelt es sich um ein Kompressionssyndrom des Nervus medianus im Bereich des Handgelenkes. Etwa 10\% der Bevölkerung sind betroffen, wobei mehr Männer als Frauen an diesem Krankheitsbild leiden (Böttner 2010). Der Nervus medianus zieht vom Unterarm aus durch den Karpaltunnel in die Handinnenfläche. Der Boden und die Wände des Karpaltunnels setzen sich aus mehreren Handwurzelknochen zusammen, während das Dach durch das Retinaculum flexorum, ein derbes, bindegewebiges Band, gebildet wird. Neben dem Nervus medianus ziehen auch die Fingerbeugesehnen durch den Karpaltunnel (Rudigier 2006). Der Nerv teilt sich im Kanal, oder distal des Kanals, in seine sensiblen und motorischen Anteile zur Versorgung der Hand auf. Ursache eines KTS ist eine Druckerhöhung im Karpaltunnel, die vielfältigste Gründe haben kann. In der Mehrzahl der Fälle liegt dem KTS eine 
Schrumpfung bzw. eine Degeneration des Retinaculum flexorum, eine Vermehrung oder Schwellung des synovialen Gleitlagers der Sehnen oder eine Veränderung der umgebenden knöchernen Strukturen zu Grunde (Rudigier 2006). Die Patienten berichten von einem Taubheitsgefühl oder von Schmerzen im Bereich des Versorgungsgebietes des Nervus medianus. Die Beschwerden treten vor allem nachts auf. Neben den wegweisenden Symptomen zeigt sich im fortgeschrittenen Stadium eine Atrophie des Daumenballens. Klinische Tests, wie beispielsweise das Hoffmann-Tinel-Zeichen oder der Phalen-Test, ermöglichen dem erfahrenen Untersucher bereits die eindeutige Diagnose eines KTS. Zudem wird die Diagnose häufig durch die Messung einer herabgesetzten Nervenleitgeschwindigkeit untermauert (Böttner 2010). In der handchirurgischen Abteilung des St. Vincenz Krankenhauses ist die offen chirurgische Spaltung des Retinaculums bis in die angrenzende Palmaraponeurose hinein der Standard zur Therapie des KTS. Durch die Entlastung des Nervs können sich so die präoperativen Beschwerden nahezu vollständig zurückbilden. Je nach Befund und Krankheitsstadium, in dem der Patient operiert wird, sind jedoch bleibende sensible und motorische Störungen möglich (Rudigier 2006).

Bei Ringbandstenosen (RBS) kommt es zu Engpässen der Ringbänder, die zur Führung der Beugesehnen im Bereich der Hohlhand und der Finger notwendig sind. Die Sehne kann bei Bewegungen des Fingers nicht mehr frei durch das Ringband gleiten. Im frühen Stadium der RBS ist diese durch das klinische Bild des „schnellenden Fingers“ (Digitus saltans) gekennzeichnet. Hierbei kann der Patient die beim Beugen oder Strecken im Ringband hängen gebliebene Sehne selbstständig durch Zug oder Druck am betroffenen Finger befreien und der Finger schnellt in die Streckstellung zurück. Ringbandstenosen können angeboren oder erworben sein. Die erworbenen RBS sind häufiger als die Angeborenen und treten meist im Alter von 40 bis 60 Jahren auf, wobei Frauen häufiger betroffen sind als Männer (Böttner 2010, Rudigier 2006). Bei den angeborenen RBS ist das Ringband zu klein für die durchlaufende Sehne, hier ist in den meisten Fällen der Daumen betroffen. Im Erwachsenenalter kommt es durch altersbedingte Veränderungen oder zu hohe Belastung zu einer Volumenzunahme der Sehne oder des umgebenden Gewebes. Bei den älteren Patienten ist meistens das erste Ringband betroffen, es liegt im Bereich der Köpfchen der Mittelhandknochen. In der klinischen Untersuchung ist die pathologische Verdickung der Sehne meist bei passiver Bewegung des Fingers palmarseitig im Bereich des Grundgelenkes des erkrankten Fingers tastbar (Rudigier 2006). Standardverfahren in unserem Haus ist die chirurgische Spaltung des betroffenen 
Ringbandes und Befreiung der Sehnen von gegebenenfalls stenosierendem Gleitgewebe. Um ein erneutes Verkleben der durchtrennten Enden des Ringbandes zu verhindern, und so einem Rezidiv vorzubeugen, wird ein Teil des Ringbandes reseziert. Eine vollständige Heilung ist nur möglich, solange es durch die eingeschränkte Beweglichkeit noch nicht zu Kontrakturen gekommen ist. Je früher die Indikation zur Operation gestellt wird, desto besser ist das Outcome (Rudigier 2006).

Ein Teil der untersuchten Patienten bedurfte der Versorgung einer Fraktur an der Hand. Die Osteosynthesen, die im Rahmen der Studie durchgeführt wurden, erfolgten vor allem bei Frakturen im Bereich der Handwurzel (Os scaphoideum) und der Mittelhandknochen (Os metacarpale D1 und D5). Die Frakturen wurden je nach Art des Knochenbruchs mittels intramedullärer Drähte, Platten- oder Schraubenosteosynthesen versorgt. 


\subsection{Einteilung der Messzeitpunkte}

Die gesamte Messung bestand aus sieben Messzeitpunkten zu denen die genannten Parameter (Herzfrequenz, Blutdruck, Sauerstoffsättigung, Hautwiderstand, expiratorischer $\mathrm{CO}_{2}$-Partialdruck, BIS-Wert, Hauttemperatur, expiratorische SevofluranKonzentration und MAC-Wert) dokumentiert wurden.

\begin{tabular}{l|cc}
\hline \multicolumn{1}{c}{ Messzeitpunkt } & Allgemeinanästhesie & axPlex \\
\hline \hline $\mathbf{T}_{\text {Ausgangswert }}$ & Messung im AWR & Messung im AWR bis Plex-Anlage \\
$\mathbf{T}_{\text {Narkosebeginn }}$ & Messung im OP vor Narkoseeinleitung & Plexus Anlage \\
$\mathbf{T}_{\text {Blutsperre }}$ & Anlage Blutsperre bis Schnitt (exklusive Schnitt) \\
$\mathbf{T}_{\text {Schnitt }}$ & Schnitt bis einschließlich Öffnung der Blutsperre \\
$\mathbf{T}_{\text {OP-Ende }}$ & OP Ende bis Pat wach ansprechbar \\
$\mathbf{T}_{\text {Aufwachraum }}$ & (inkl. Ende) \\
$\mathbf{T}_{\text {Normalstation }}$ & Messung im AWR post OP \\
\hline
\end{tabular}

Tabelle 2: Übersicht der Messzeitpunkte

$\mathrm{T}_{\text {Ausgangswert }}$ Während des ersten Messzeitpunkts lag der Patient ruhig im Aufwachraum. Die so ermittelten Werte wurden für jeden Patienten gemittelt und als individuelle Ausgangswerte festgelegt. Sie dienten als Grundlage, um die Veränderungen in den anderen Zeitpunkten berechnen und auswerten zu können.

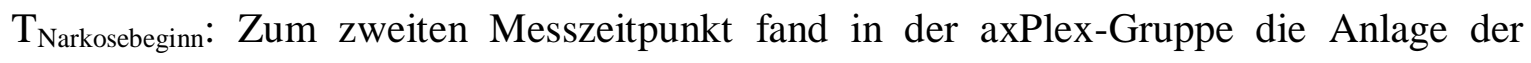
axillären Plexusblockade unter sonographischer Kontrolle statt. Die Patienten der ITNGruppe wurden bereits in den OP gebracht. Hier wurde ihre Reaktion auf die unmittelbar bevorstehende Narkoseeinleitung ermittelt. 
$\mathrm{T}_{\text {Blutsperre: }}$ In beiden Gruppen wurden die Werte von der Anlage der Blutsperre, durch die Kollegen der Handchirurgie, bis unmittelbar vor dem Hautschnitt notiert. In der ITNGruppe erfolgte hier die Narkoseeinleitung.

$\mathrm{T}_{\text {Schnitt: }}$ Der vierte Messzeitpunkt umfasste die Phase der Operation vom Hautschnitt bis zur Öffnung der zuvor angelegten Blutsperre.

$T_{\text {OP-Ende: }}$ Der fünfte Messzeitpunkt erstreckte sich vom Ende der Operation bis zum Ende der Messung im OP. Als Endpunkt für die Messung in der ITN-Gruppe wurde der Zeitpunkt festgelegt, an dem der Patient wieder wach und orientiert war.

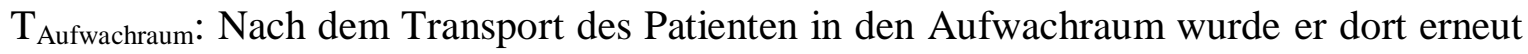
an das Monitoringsystem angeschlossen und die Werte erneut im Abstand von fünf Minuten notiert. Zudem wurde anhand einer NRS-Skala das perioperative Schmerzempfinden dokumentiert.

$\mathrm{T}_{\text {Normalstation: }}$ Auf der peripheren Station erfolgte vor Entlassung des Patienten die Messung von Hauttemperatur, ESG und postoperativem Schmerzempfinden mittels der NRS-Skala. 


\section{3 Überwachung}

\subsubsection{Basismonitoring}

Bei jedem Patienten erfolgte standardmäßig die Überwachung der Vitalparameter Blutdruck, Herzfrequenz, und Sauerstoffsättigung, prä- und postoperativ im Aufwachraum mit dem Propaq Encore - Monitor (Welch Allyn, New York, USA) intraoperativ mit den Messgeräten des Narkosegerätes ZEUS (Firma Dräger, Lübeck, Deutschland). In der Gruppe der Allgemeinanästhesie wurde zudem der expiratorische $\mathrm{pCO}_{2}$ ermittelt. Zur Messung der Narkosetiefe während der Vollnarkose kam das BIS ${ }^{\circledR}$ Monitoring (Aspect Medical Systems, Norwood, USA) zur Anwendung. Die Hauttemperatur wurde mit dem bosotherm medical - Thermometer boso (Firma Bosch + Sohn, Jungingen, Deutschland) an der Hand gemessen, an der auch die ESG Messung erfolgte. Um die Sympathikusaktivität mittels des Elektrosympathikographen zu bestimmen, brachten wir bei jedem Patienten an der nicht zu operierenden Hand je eine $\mathrm{Ag} / \mathrm{AgCl}$-Elektrode (ASF40C, Asmuth GmbH Medizintechnik, Minden, Germany) palmarseitig am Endglied von Daumen und Zeigefinger an (s. Abbildung 2).

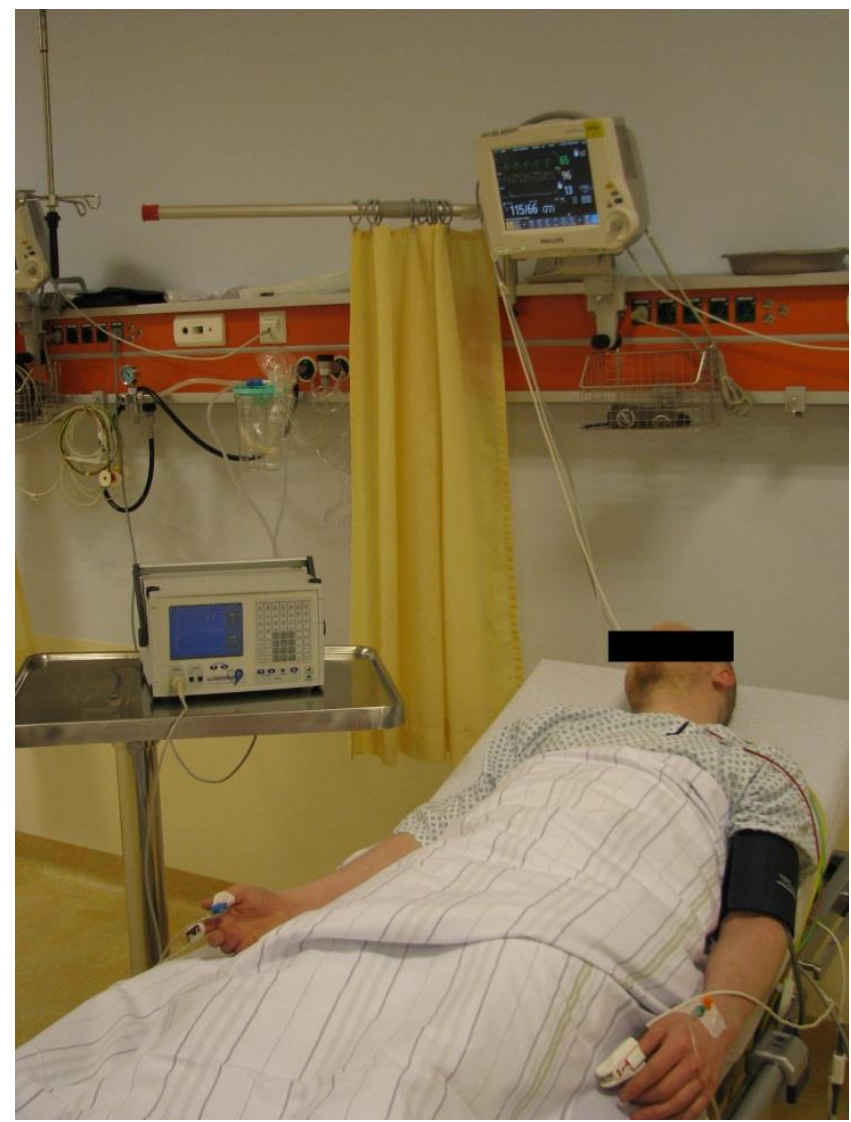

Abbildung 1: Basismonitoring im Aufwachraum

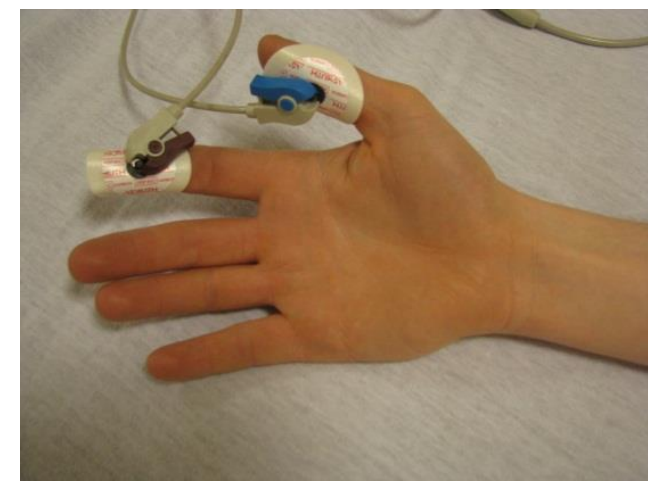

Abbildung 2: Anschluss des ESG 


\subsubsection{Elektrosympathikograph}

Zur Messung des Hautwiderstandes und damit der Aktivität des Sympathikus, wurde das Tischgerät ESG 1001 verwendet (ESG 1001 Monitor-System, Ingenieurbüro Dr. Janitzki, Altenbeken, Germany)(s. Abbildung 3). Die Methode zur Registrierung des Elektrosympathikogramms entwickelte Dr. Janitzki im Fachbereich Nachrichtentechnik der Universität Paderborn (Janitzki 2010a). Als Sensoren dienen zwei $\mathrm{Ag} / \mathrm{AgCl}$-Elektroden mit einer Oberfläche von jeweils ca. $1 \mathrm{~cm}^{2}$. Bei einer definierten Stromdichte von $<2 \mu \mathrm{A} / \mathrm{cm}^{2}$ wird über die Sensoren Gleichstrom abgegeben. Das ESG registriert den Spannungsabfall des abgegebenen Gleichstroms über den jeweiligen Messorten. Widerstandsänderungen werden nahezu ausschließlich im Bereich der Elektroden erfasst. Ursächlich dafür sind die annähernd senkrecht ins Körperinnere führenden Strombahnen, die an den Sensoren ihren Ursprung haben. Der Messbereich liegt zwischen $5 \mathrm{kOhm}$ und $2 \mathrm{MOhm}$. Am Monitor des ESG werden die Widerstände als ganze kOhm angezeigt (Janitzki 2010a). Die registrierten Spannungsabfälle werden im Abstand von $200 \mathrm{~ms}$ aufgezeichnet. Nach der Registrierung folgt eine analoge Signalaufbereitung und Filterung. Um die Störanfälligkeit möglichst gering zu halten, werden die Daten frühzeitig digitalisiert und im internen Speicher des PCs, der sich im ESG befindet, abgelegt (Winterhalter 2007). Ähnlich wie beim BIS wurde auch beim ESG der genaue Ablauf der Datenverarbeitung durch den Hersteller nicht freigegeben. Am Monitor des ESG werden im Abstand von 5 Sekunden die aktuellen tonischen Werte des Hautwiderstandes angezeigt, in denen phasische Veränderungen auf äußere Reize enthalten sind. Die genaue Erläuterung der tonischen und phasischen Widerstände sowie weitere Details zur Sympathikographie finden sich im Kapitel 1.2. Die Anzeige erfolgt als tonisches Elektrosympathikogramm (tonic ESG). Die kOhm-Werte werden als Kurve in Abhängigkeit von der Zeit dargestellt. Diese Darstellung birgt eine Besonderheit im Gegensatz zu anderen Monitorsystemen der Anästhesie, bei denen meist eine hohe Aktivität durch entsprechend hohe Monitorwerte dargestellt wird. Beim ESG hingegen wird eine hohe Aktivität des Sympathikus, die zu einer Abnahme des Hautwiderstandes führt, mit niedrigen $\mathrm{kOhm}$-Werten dargestellt. 


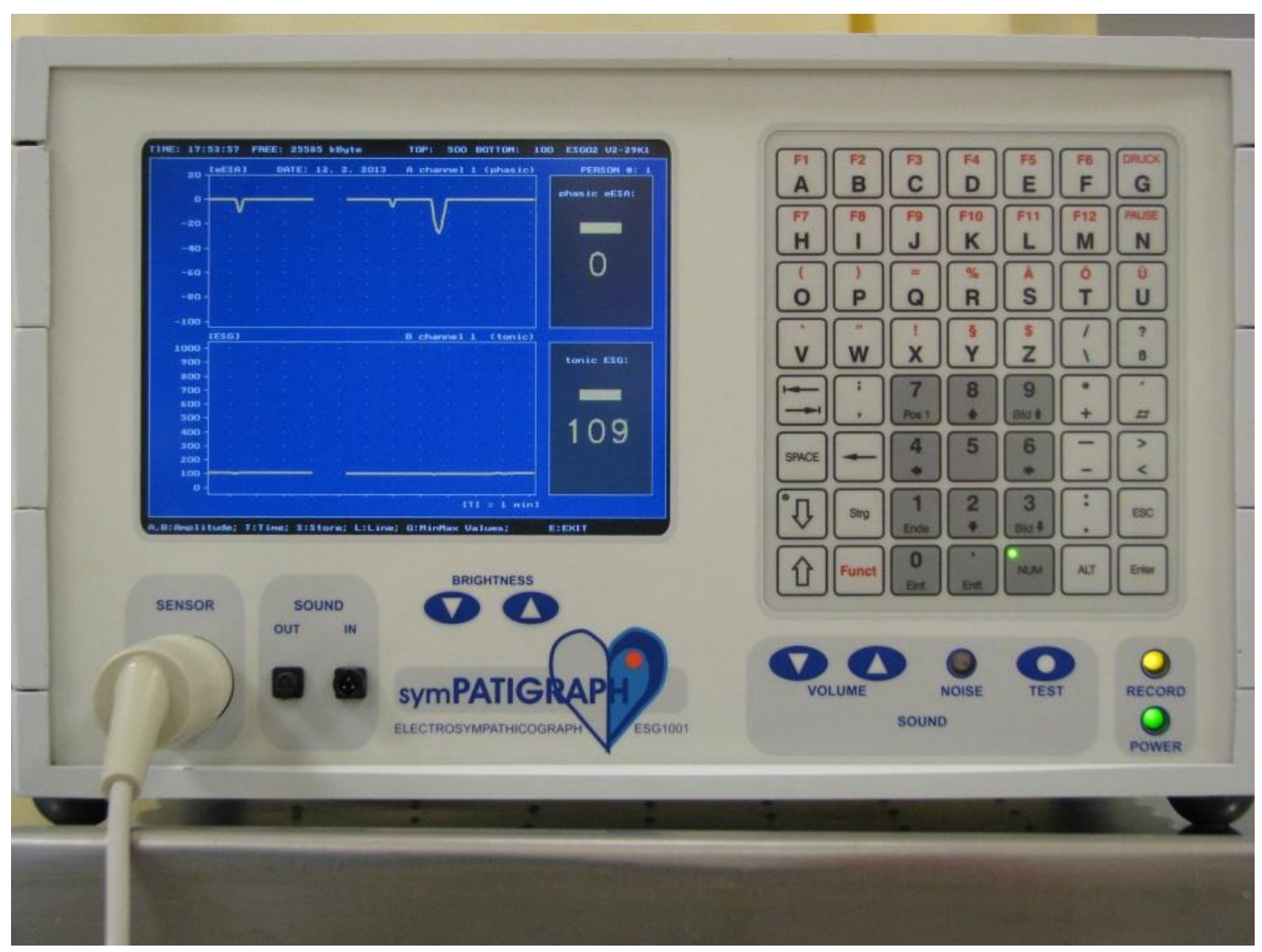

Abbildung 3: Tischgerät ESG 1001 


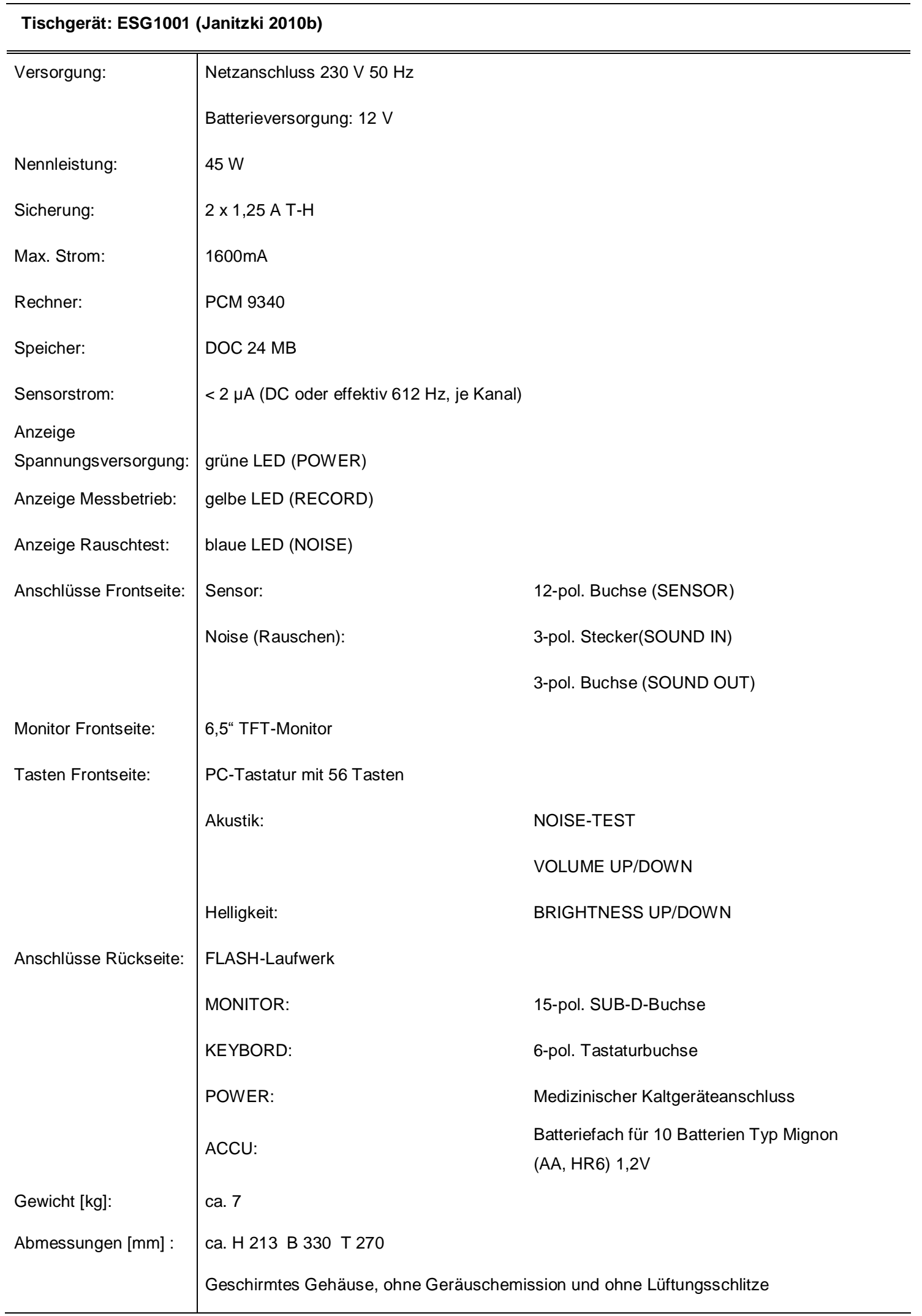

Als Sensoren des ESG und beim Basismonitoring wurden Ag/AgCl-Elektroden (ASF40C, Asmuth GmbH Medizintechnik, Minden, Germany) verwendet, die in unserer Klinik standardmäßig zur perioperativen Ableitung des Patienten EKGs verwendet werden. 


\subsubsection{Bispectral-Index (BIS)}

Beim BIS-Monitoring handelt es sich um ein System zur Überwachung der Narkosetiefe. Grundlage ist das EEG. Die EEG-Signalaufnahme erfolgte über Klebeelektroden (BIS ${ }^{\circledR}$ QUATRO, Aspect Medical Systems, Norwood, USA) die auf der Stirn des Patienten angebracht werden. Dieses Signal wird in einem Analog-DigitalKonverter umgewandelt und geräteintern wird der BIS-Wert berechnet. In dieser Studie wurde der BIS ${ }^{\circledR}$-Monitor der Firma Aspect (Aspect Medical Systems, Norwood, USA) verwendet. Der BIS-Wert ist dimensionslos und liegt zwischen 0 und 100. Bei einem BISWert von 0 liegt keine EEG-Aktivität vor, beträgt der Wert 100, so ist der Patient wach. In dieser Studie wurde bei den Fällen mit Allgemeinanästhesie ein Wert zwischen 40 bis 60 angestrebt, da in diesem Bereich jeweils eine ausreichende Schlaftiefe vorliegt und keine Awareness $\mathrm{zu}$ erwarten ist (Wilhelm und Kreuer 2003). Auch möglicherweise zu erwartende ungünstige neurovegetative und kardiovaskuläre Effekte bei größeren individuell unterschiedlichen Anästhesietiefen, mit eventuell sogar totaler neurovegetativer Blockade, können durch das BIS-Monitoring vermieden werden.

\subsection{Narkoseform}

\subsubsection{Allgemeinanästhesie}

Im Operationssaal wurde der Patient an das Monitoring angeschlossen. Es folgte ein initialer Messzeitpunkt, in dem alle beschriebenen Untersuchungswerte ermittelt wurden. Nach ausreichender Präoxygenierung wurden folgende Medikamente zur Narkoseeinleitung verwendet:

Fentanyl (Fentanyl ${ }^{\circledR}$-Janssen, Janssen-Cilag, Neuss, Deutschland) $(1-5 \mu \mathrm{g} / \mathrm{kgKG})$

Propofol (Propofol ${ }^{\circledR}$-Lipuro $10 \mathrm{mg} / \mathrm{ml}$ (1\%), Braun, Melsungen, Deutschland) bis zu einem BIS-Wert $<60$ bei maximal 2,5 mg/kgKG

Bei ausreichender Narkosetiefe des Patienten fand eine Atemwegssicherung mittels einer Larynxmaske (Ambu ${ }^{\circledR}$ Aura Once ${ }^{\mathrm{TM}}$, Ambu Inc., Glen Burnie, USA) statt. Die Messung der Narkosetiefe erfolgte mit dem BIS - Monitoring (Aspect Medical Systems, Norwood, USA). Die Anästhesie wurde mit Sevofluran (Sevorane ${ }^{\circledR}$, Abbot GmbH \& Co. KG, Wiesbaden, Deutschland) in angepasster exspiratorischer Konzentration aufrechterhalten 
(MAC 0,5-3 Vol.-\%). Die Beatmung und Überwachung erfolgte mit dem Narkosegerät ZEUS (Firma Dräger, Lübeck, Deutschland). EKG, Sauerstoffsättigung $\left(\mathrm{S}_{\mathrm{a}} \mathrm{O}_{2}\right)$ und expiratorischer $\mathrm{pCO}_{2}$ wurden kontinuierlich und der Blutdruck alle 5 min aufgezeichnet. Bei Bedarf wurden während der Narkose zusätzliche Analgetika wie Piritramid (Dipidolor $^{\circledR}$, Janssen-Cilag, Neuss, Deutschland) 3,75-7,5 mg i.v., Metamizol Natrium $1 \mathrm{H}_{2} \mathrm{O}$ (Novaminsulfon-ratiopharm ${ }^{\circledR}$, Ratiopharm, Ulm, Deutschland) 2,5 g als Kurzinfusion oder Paracetamol (Perfalgan ${ }^{\circledR}$, Bristol-Myers Squibb, München, Deutschland) $1 \mathrm{~g}$ als Kurzinfusion verabreicht.

\subsubsection{Axilläre Plexusanästhesie}

Nach Auslagerung des zu operierenden Arms, gründlicher Desinfektion und steriler Abdeckung erfolgte die ultraschallgesteuerte Anlage der axillären Plexusanästhesie. Zur Sonographie wurde das Micro Max Ultrasound System der Firma SonoSite ${ }^{\circledR}$ (SonoSite ${ }^{\circledR}$ Inc., Bothell, USA) verwendet.

Eine $40 \mathrm{ml}$ Lösung bestehend aus $20 \mathrm{ml}$ Ropivacain 0,75\% (Naropin® 7,5 mg/ml, AstraZeneca GmbH, Wedel, Deutschland), $10 \mathrm{ml}$ Prilocain $1 \%$ (Xylonest ${ }^{\circledR}$, AstraZeneca GmbH, Wedel, Deutschland) und $10 \mathrm{ml}$ Prilocain 2\% (Xylonest ${ }^{\circledR}$, AstraZeneca GmbH, Wedel, Deutschland) wurde injiziert. Unter sonographischer Kontrolle wurden $5 \mathrm{ml}$ der Lösung mittels einer Stimuplex ${ }^{\circledR}$ A - Nadel (22 G x 2 “, 0,7 x 50 mm) der Firma Braun ${ }^{\circledR}$ (Braun, Melsungen, Deutschland) am Nervus musculocutaneus, und die restlichen $35 \mathrm{ml}$ zu gleichen Teilen an Nervus ulnaris, Nervus radialis und Nervus medianus platziert.

\subsubsection{Schmerztherapie}

Das peri- und postoperative Schmerzempfinden der Patienten wurde mit einer numerischen Ratingskala (NRS-Skala) von null bis zehn bestimmt. Kein Schmerzempfinden wurde mit null und maximal vorstellbarer Schmerz mit zehn bewertet. Kein Schmerz

\begin{tabular}{|l|l|l|l|l|l|l|l|l|l|l|}
\hline 0 & 1 & 2 & 3 & 4 & 5 & 6 & 7 & 8 & 9 & 10 \\
\hline
\end{tabular}

max. Schmerz

Zur postoperativen Schmerztherapie wurden Piritramid (Dipidolor®, Janssen-Cilag, Neuss, Deutschland) 3,75-7,5 mg i.v., Metamizol Natrium $1 \mathrm{H}_{2} \mathrm{O}$ (Novaminsulfonratiopharm®, Ulm, Deutschland) 2,5 g als Kurzinfusion oder als Tropfen p.o., Paracetamol (Perfalgan®, Bristol-Myers Squibb, München, Deutschland) $1 \mathrm{~g}$ als Kurzinfusion, Pethidinhydrochlorid (Dolantin®, Sanofi aventis, Frankfurt am Main, 
Deutschland) 25 mg i.v. oder Ibuprofen (Ibuhexal®, Hexal, Holzminden, Deutschland) 400-600 mg p.o. verabreicht.

\subsubsection{Statistische Auswertung}

Nach der Datenerhebung wurden die Rohdaten in eine Excel-Tabelle eingegeben. Die statistische Auswertung sowie die graphische Darstellung der ermittelten Daten in Form von Boxplots erfolgte mit dem Statistikprogramm Statistica ${ }^{\circledR}$ Version 9.0 (Statistica 9.0, StatSoft Europe GmbH, Hamburg). Für alle statistischen Tests wurde ein p-Wert $<0,05$ als statistisch signifikant angenommen. Vor Studienbeginn wurde eine Poweranalyse durchgeführt. Grundlage dieser Analyse waren die Daten der Arbeit von Knabe, Götte und Janitzki (Knabe et al. 1997). Bei zwei Grundgesamtheiten (ESGMittelwert $1=500$ und ESG-Mittelwert 2=250, Standardabweichung $=250$, Teststärke $=0,9$ ) wurden je 23 Patienten in jeder Gruppe benötigt, um einen Fehler < 0,05 auszuschliessen. Die Überprüfung der Daten auf Normalverteilung erfolgte mit dem Kolmogorov-Smirnov-Test. Bei Annahme von normalverteilten Daten wurden ordinalskalierte Ergebnisse als Mittelwert +/- Standardabweichung angegeben. Die Überprüfung auf Unterschiede zwischen den beiden Studiengruppen erfolgte dann mit dem t-Test für unverbundene Stichproben. Bei Ablehnung der Normalverteilung wurden die Ergebnisse als Median und Bereich angegeben. Die Überprüfung auf signifikante Unterschiede bei ordinalskalierten Daten erfolgte dann mit dem Mann-Whitney-U-Test bei nicht verbundenen Daten und mit dem Wilcoxon-Matched-Pair-Test bei verbundenen Daten. Kategoriale Daten wurden unabhängig von ihrer Verteilung als prozentuale Verteilung angegeben. Die statistische Testung erfolgte mit dem Fishers-Exact-Test. 


\section{Ergebnisse}

\subsection{Patienten}

In die Studie wurden insgesamt 46 Patientinnen und Patienten eingeschlossen, die sich unter Allgemeinanästhesie (ITN) oder Regionalanästhesie (axPlex) einer handchirurgischen Operation unterzogen. Die Analyse der anthropometrischen Daten ist in Tabelle 3 dargestellt.

\begin{tabular}{|c|c|c|c|}
\hline Variable & $\begin{array}{l}\text { ITN } \\
n=23\end{array}$ & $\begin{array}{c}\text { axPlex } \\
n=23\end{array}$ & p-Wert \\
\hline Alter [Jahre] & $21-76(51)$ & $27-66(52)$ & 0,73 \\
\hline Geschlecht [m/w] [\%] & $35 / 65$ & $61 / 39$ & 0,07 \\
\hline Größe [cm] & $152-188(168)$ & $155-190(174)$ & 0,09 \\
\hline Gewicht [kg] & $42-111(74)$ & $62-110(78)$ & 0,46 \\
\hline BMI & $18,2-40,3(26,4)$ & $20,9-34,9(25,5)$ & 0,97 \\
\hline ASA I [\%] & 35 & 52 & - \\
\hline ASA II [\%] & 65 & 48 & - \\
\hline \multicolumn{4}{|l|}{ Vorerkrankungen } \\
\hline Hypertonus [\%] & 30 & 26 & - \\
\hline Reflux [\%] & 8,7 & 8,7 & - \\
\hline Neurodermitis [\%] & 0 & 4,3 & - \\
\hline Asthma bronchiale [\%] & 0 & 13 & - \\
\hline Absolute Arrhythmie [\%] & 4,3 & 4,3 & - \\
\hline chron. Bronchitis [\%] & 0 & 4,3 & - \\
\hline D.m. Typ II [\%] & 4,3 & 0 & - \\
\hline \multicolumn{4}{|l|}{ Perioperative Zeiten } \\
\hline Schnitt-Naht-Zeit [min] & $9-76(24)$ & $7-75(24)$ & 1,0 \\
\hline Aufwachraum [min] & $19-90(36)$ & $10-91(28)$ & 0,009 \\
\hline Krankenhaus [h] & $5,7-54,45(10,5)$ & $5,71-30,4(8,8)$ & 0,05 \\
\hline
\end{tabular}

Tabelle 3: Analyse der anthropometrischen Daten 
Die Auswertung von Alter, Geschlecht, Größe, Gewicht und Body Mass Index ergab keinen statistisch signifikanten Unterschied zwischen beiden Gruppen. Die Analyse der perioperativen Zeiten - Schnitt/Naht-Zeit $(p=1,0)$ und Dauer des Aufenthalts im Krankenhaus $(p=0,05)-$ konnte ebenfalls keinen signifikanten Unterschied zwischen beiden Untersuchungsgruppen nachweisen. Lediglich beim Vergleich des Aufenthalts im Aufwachraum ( $\mathrm{p}=0,009)$ zeigte sich eine signifikant längere Aufenthaltsdauer der Patienten der ITN-Gruppe (Median=36) im Vergleich $\mathrm{zu}$ den Patienten in der axPlex-Gruppe $($ Median $=28)($ siehe Tabelle 3$)$.

Um eine ausreichende Narkosetiefe auf Seiten der Patienten der ITN-Gruppe zu gewährleisten, wurde ein BIS-Wert von 40-60 angestrebt. Die statistische Prüfung der intraoperativen BIS-Werte aller Patienten der ITN-Gruppe ergab einen Wert von $52 \pm 5$.

\subsection{Schmerzempfinden und Schmerztherapie}

Die statistische Auswertung der peri- und postoperativen NRS-Werte beider Studiengruppen zeigte in beiden Phasen signifikant höhere Schmerzen in der ITN-Gruppe als in der axPlex-Gruppe (siehe Tabelle 4).

\begin{tabular}{l|ccc}
\hline NRS & $\begin{array}{c}\text { ITN } \\
\mathrm{n}=23\end{array}$ & $\begin{array}{c}\text { axPlex } \\
\mathrm{n}=23\end{array}$ & p-Wert \\
\hline \hline Aufwachraum & $0-8(0)$ & $0-0(0)$ & 0,001 \\
Normalstation & $0-5(2,5)$ & $0-5(0)$ & 0,01 \\
\hline
\end{tabular}

Tabelle 4: peri- und postoperative Schmerzen 
Tabelle 5 zeigt die Auswertung des Schmerzmittelbedarfs beider Untersuchungsgruppen.

\begin{tabular}{l|ccc}
\hline & ITN & axPlex & p-Wert \\
\hline \hline Analgetika intraop & & & \\
Fentanyl [mg] & $0,1-0,2(0,15)$ & 0 & 0,000000 \\
Piritramid [mg] & $0-6(0)$ & 0 & 0,024 \\
Metamizol Natrium 1 $\mathrm{H}_{2} \mathrm{O}$ [g] & $0-2,5(2,5)$ & 0 & 0,000000 \\
Paracetamol [g] & $0-1(0)$ & 0 & 0,037 \\
Analgetika postop & & & \\
Piritramid [mg] & $0-7,5(0)$ & 0 & 0,002 \\
Metamizol Natrium 1 $\mathrm{H}_{2} \mathrm{O}$ [g] & $0-0,75(0)$ & 0 & 0,322 \\
Paracetamol [g] & $0-1(0)$ & 0 & 0,037 \\
Pethidinhydrochlorid [mg] & $0-25(0)$ & 0 & 0,323 \\
Ibuprofen [mg] & $0-600(0)$ & 0 & 0,323 \\
\hline
\end{tabular}

Tabelle 5: intra- und postoperativer Schmerzmittelbedarf

Die Auswertung des intraoperativen und postoperativen Schmerzmittelbedarfes ergab einen signifikant höheren Bedarf in der ITN-Gruppe.

\subsection{Basismonitoring}

$\mathrm{Zu}$ Beginn der Messungen wurden zum Messzeitpunkt $\mathrm{T}_{\text {Ausgangswert }}$ die Ausgangswerte in beiden Gruppen bestimmt. Die Ausgangswerte definierten den Grundtonus des Patienten. Zur Auswertung erfolgte ein Vergleich der im weiteren Verlauf der Studie ermittelten Daten von Herzfrequenz, mittlerem arteriellen Druck (MAD), ESG-Messung und Hauttemperatur mit dem jeweiligen Ausgangswert. Veränderungen wurden als prozentuale Abweichung vom Ausgangswert angegeben. Bei der Auswertung der Temperaturmessung wurden die Veränderungen in Grad Celsius errechnet. Die Tabelle 6 zeigt die ermittelten Daten und die statistische Auswertung über die gesamte Messung. 


\begin{tabular}{|c|c|c|c|}
\hline & ITN & axPlex & $p$-Wert \\
\hline \multicolumn{4}{|l|}{$\mathbf{T}_{\text {Ausgangswert }}$} \\
\hline $\mathrm{MAD}[\mathrm{mmHg}]$ & $67-111(90)$ & $74-127(93)$ & 0,19 \\
\hline $\mathrm{HF}\left[\mathrm{min}^{-1}\right]$ & $49-98(65)$ & $49-107$ (67) & 0,79 \\
\hline Temp $\left[{ }^{\circ} \mathrm{C}\right]$ & $27,8-35,3(31,3)$ & $28,0-35,2(31,5)$ & 0,72 \\
\hline Hautwiderstand [kOhm] & $75-746(281)$ & $66-392(180)$ & 0,02 \\
\hline \multicolumn{4}{|l|}{$\mathbf{T}_{\text {Narkosebeginn }}$} \\
\hline MAD [mmHg] & $69-131(100)$ & $73-128(93)$ & 0,25 \\
\hline MAD [\% vom Ausgangswert] & $-10-+36(+12)$ & $-11-+8(+2)$ & 0,0001 \\
\hline $\mathrm{HF}\left[\min ^{-1}\right]$ & $49-105$ (73) & $51-109(68)$ & 0,3 \\
\hline HF [\% vom Ausgangswert] & $-13-+30(+4)$ & $-7-+11(+1)$ & 0,15 \\
\hline Hautwiderstand [kOhm] & $58-470(122)$ & $50-543(143)$ & 0,91 \\
\hline Hautwiderstand [\% vom Ausgangswert] & $-80-+9(-52)$ & $-45-+40(-24)$ & 0,0001 \\
\hline Temp $\left[{ }^{\circ} \mathrm{C}\right]$ & $27,9-34(31,2)$ & $26,6-34,9(31,2)$ & 0,48 \\
\hline Temp [Diff. vom Ausgangswert in ${ }^{\circ} \mathrm{C}$ ] & $-4-+2,4(-1,3)$ & $-2,5-+0,4(-0,6)$ & 0,19 \\
\hline \multicolumn{4}{|l|}{$\mathbf{T}_{\text {Blutsperre }}$} \\
\hline MAD [mmHg] & $55-107(71)$ & $83-126(104)$ & 0,000000 \\
\hline MAD [\% vom Ausgangswert] & $-50-+13(-19)$ & $-6-+31(+11)$ & $<0,001$ \\
\hline $\mathrm{HF}\left[\mathrm{min}^{-1}\right]$ & $42-82(58)$ & $53-106(65)$ & 0,008 \\
\hline HF [\% vom Ausgangswert] & $-37-+22(-12)$ & $-11-+25(+5)$ & $<0.001$ \\
\hline Hautwiderstand [kOhm] & $134-825(295,5)$ & $40-182(105)$ & 0,000001 \\
\hline Hautwiderstand [\% vom Ausgangswert] & $-54-+137(14)$ & $-69-+2(-41)$ & $<0,001$ \\
\hline Temp $\left[{ }^{\circ} \mathrm{C}\right]$ & $18,9-33,8(31)$ & $25,2-34,5(29,4)$ & 0,5 \\
\hline Temp [Diff. vom Ausgangswert in ${ }^{\circ} \mathrm{C}$ ] & $-11-+2(-1,4)$ & $-5,3-+2(-2,3)$ & 0,03 \\
\hline \multicolumn{4}{|l|}{$\mathbf{T}_{\text {Schnitt }}$} \\
\hline MAD [mmHg] & $60-93(79)$ & $80-119(99)$ & 0,000000 \\
\hline MAD [\% vom Ausgangswert] & $-30-+10(-8)$ & $-8-+23(+8)$ & $<0,001$ \\
\hline $\mathrm{HF}\left[\min ^{-1}\right]$ & $43-89(54,5)$ & $55-101(66)$ & 0,099 \\
\hline HF [\% vom Ausgangswert] & $-35-+55(-15)$ & $-10-+22(+2)$ & $<0,001$ \\
\hline ESG [kOhm] & $181-823(381)$ & 4-271 (129) & 0,000000 \\
\hline ESG [\% vom Ausgangswert] & $-36-+227(+36)$ & $-98-+29(-34)$ & $<0,001$ \\
\hline Temp $\left[{ }^{\circ} \mathrm{C}\right]$ & $27,5-34,5(31,9)$ & $24,9-35,6(28,6)$ & 0,005 \\
\hline Temp [Diff. vom Ausgangswert in ${ }^{\circ} \mathrm{C}$ ] & $-1,4-+2,8(+0,1)$ & $-5,6-+4,7(-2,5)$ & $<0,001$ \\
\hline \multicolumn{4}{|l|}{ ToP-Ende } \\
\hline $\mathrm{MAD}[\mathrm{mmHg}]$ & $76-108(85)$ & $78-123(100)$ & 0,0002 \\
\hline MAD [\% vom Ausgangswert] & $-26-+40(+2)$ & $-4-+25(+8)$ & 0,006 \\
\hline $\mathrm{HF}\left[\mathrm{min}^{-1}\right]$ & $45-88(66)$ & $52-99(65)$ & 0,86 \\
\hline HF [\% vom Ausgangswert] & $-22-+57(-4)$ & $-14-+27(+3)$ & 0,36 \\
\hline ESG [kOhm] & $178-944(387)$ & $40-314(109)$ & 0,000000 \\
\hline ESG [\% vom Ausgangswert] & $-32-+189(+38)$ & $-74-+60(-32)$ & $<0,001$ \\
\hline Temp $\left[{ }^{\circ} \mathrm{C}\right]$ & $27,7-34,9(32,4)$ & $25,2-35,2(28,4)$ & 0,001 \\
\hline Temp [Diff. vom Ausgangswert in ${ }^{\circ} \mathrm{C}$ ] & $-3,5-+2,8(0)$ & $-6,3-+4,3(-3,8)$ & $<0,001$ \\
\hline \multicolumn{4}{|l|}{$\mathbf{T}_{\text {Aufwachraum }}$} \\
\hline MAD [mmHg] & $72-108(90,5)$ & $73-115(94)$ & 0,15 \\
\hline MAD [\% vom Ausgangswert] & $-22-+25(-7)$ & $-10-+20(+3)$ & 0,76 \\
\hline $\mathrm{HF}\left[\min ^{-1}\right]$ & $48-86(68)$ & $50-98(64)$ & 0,55 \\
\hline HF [\% vom Ausgangswert] & $-19-+31(-7)$ & $-23-+20(-3)$ & 0,94 \\
\hline ESG [kOhm] & $106-1154(250,5)$ & 50-284 (107) & 0,001 \\
\hline ESG [\% vom Ausgangswert] & $-61-+168(-2)$ & $-71-+31(-32)$ & 0,005 \\
\hline Temp $\left[{ }^{\circ} \mathrm{C}\right]$ & $26,8-35,1(31)$ & $25-35,2(29,7)$ & 0,024 \\
\hline Temp [Diff. vom Ausgangswert in ${ }^{\circ} \mathrm{C}$ ] & $-3,8-+2,3(0)$ & $-6,2-+3,7(-2,7)$ & 0,001 \\
\hline \multicolumn{4}{|l|}{$\mathbf{T}_{\text {Normalstation }}$} \\
\hline ESG [kOhm] & $41-455(81)$ & $8-167(76,5)$ & 0,09 \\
\hline ESG [\% vom Ausgangswert] & $-83--18(-67)$ & $-99-+42(-65)$ & 0,81 \\
\hline $\operatorname{Temp}\left[{ }^{\circ} \mathrm{C}\right]$ & $28,4-34,5(32,7)$ & $32,1-36(34,35)$ & 0,001 \\
\hline Temp [Diff. vom Ausgangswert in ${ }^{\circ} \mathrm{C}$ ] & $-2-+4,9(+1,9)$ & $0-+5(+1,8)$ & 0,52 \\
\hline
\end{tabular}

Tabelle 6: statistische Auswertung der gesamten Messung (Diff: = Differenz) 


\subsection{Herzfrequenz}

Die ermittelten Herzfrequenzen zum Zeitpunkt $\mathrm{T}_{\text {Ausgangswert }}$ wurden als Schläge pro Minute [1/min] angegeben. In der ITN-Gruppe lagen die Werte zwischen 49 und $98 \mathrm{~min}^{-1}$ $\left(\right.$ Median $\left.=65 \mathrm{~min}^{-1}\right)$, in der axPlex-Gruppe zwischen 49 und $107 \min ^{-1}\left(\right.$ Median $\left.=67 \mathrm{~min}^{-1}\right)$. Die statistische Analyse zeigte keinen signifikanten Unterschied zwischen beiden Untersuchungsgruppen ( $\mathrm{p}=0,79)$. Bei der Auswertung der folgenden Messzeitpunkte wurden sowohl die absoluten Werte als auch die jeweilige prozentuale Abweichung vom Ausgangswert betrachtet. Zum Messzeitpunkt $\mathrm{T}_{\text {Narkosebeginn }}$ lagen die Herzfrequenzen der ITNGruppe zwischen 49 und $105 \mathrm{~min}^{-1}\left(\right.$ Median $\left.=73 \mathrm{~min}^{-1}\right)$ und der axPlex-Gruppe zwischen 51 und $109 \mathrm{~min}^{-1}\left(\right.$ Median $\left.=68 \mathrm{~min}^{-1}\right)$. Es ergaben sich für die ITN-Gruppe prozentuale Abweichungen von $-13 \%$ bis $+30 \%$ (Median $=+4 \%$ ) und für die axPlex-Gruppe von $-7 \%$ bis $+11 \%$ (Median $=+1 \%)$. Sowohl bei den absoluten Werten $(\mathrm{p}=0,3)$ als auch bei den prozentualen Abweichungen $(\mathrm{p}=0,15)$ zeigte sich kein signifikanter Unterschied. Die Auswertung der Herzfrequenzen zum Messzeitpunkt $\mathrm{T}_{\text {Blutsperre }}$ ergab auf Seiten der ITNGruppe Werte von 42 bis $82 \mathrm{~min}^{-1}\left(\right.$ Median $=58 \mathrm{~min}^{-1}$ ) und Abweichungen von $-37 \%$ bis $+22 \%$ (Median $=-12 \%$ ). In der axPlex-Gruppe lagen die Werte zwischen 53 und $106 \mathrm{~min}^{-1}$ $\left(\right.$ Median $\left.=65 \min ^{-1}\right)$ was einer Abweichung von $-11 \%$ bis $+25 \%($ Median $=+5 \%)$ entsprach . Statistisch errechnete sich ein signifikanter Unterschied zwischen beiden Gruppen bei den absoluten Werten $(\mathrm{p}=0,008)$ und bei den prozentualen Veränderungen $(\mathrm{p}<0,001)$ mit einer größeren Abweichung vom Ausgangswert auf Seiten der ITN-Gruppe. Der Messzeitpunkt $\mathrm{T}_{\text {Schnitt }}$ zeigte Herzfrequenzwerte von 43 bis $89 \mathrm{~min}^{-1}$ in der ITN-Gruppe $\left(\right.$ Median $\left.=54,5 \mathrm{~min}^{-1}\right)$ und prozentuale Abweichungen von $-35 \%$ bis $+55 \%$ (Median $=-15 \%$ ). In der axPlex-Gruppe lagen die absoluten Werte zwischen 55 und $101 \mathrm{~min}^{-1}$ $\left(\right.$ Median $\left.=66 \mathrm{~min}^{-1}\right)$ und die Differenzen vom Ausgangswert zwischen $-10 \%$ bis $+22 \%$ $($ Median $=+2 \%)$. Während die absoluten Werte keinen signifikanten Unterschied zeigten $(\mathrm{p}=0,099)$ lag der $\mathrm{p}$-Wert der prozentualen Abweichungen bei $\mathrm{p}<0,001$ und wies hier einen signifikanten Unterschied zwischen beiden Gruppen nach. Erneut lag die größere Abweichung der Herzfrequenz auf Seiten der ITN-Gruppe. Zum Zeitpunkt TOP-Ende $_{\text {und }}$

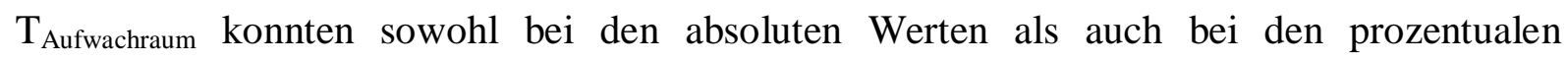
Veränderungen keine signifikanten Unterschiede zwischen beiden Gruppen nachgewiesen werden. In Abbildung 4 ist die Abweichung der gemessenen Herzfrequenz in beiden Gruppen im zeitlichen Verlauf dargestellt. Abbildung 5 zeigt zum Vergleich die prozentuale 
Abweichung der Herzfrequenz vom Ausgangswert in beiden Gruppen im Verlauf der Messung.

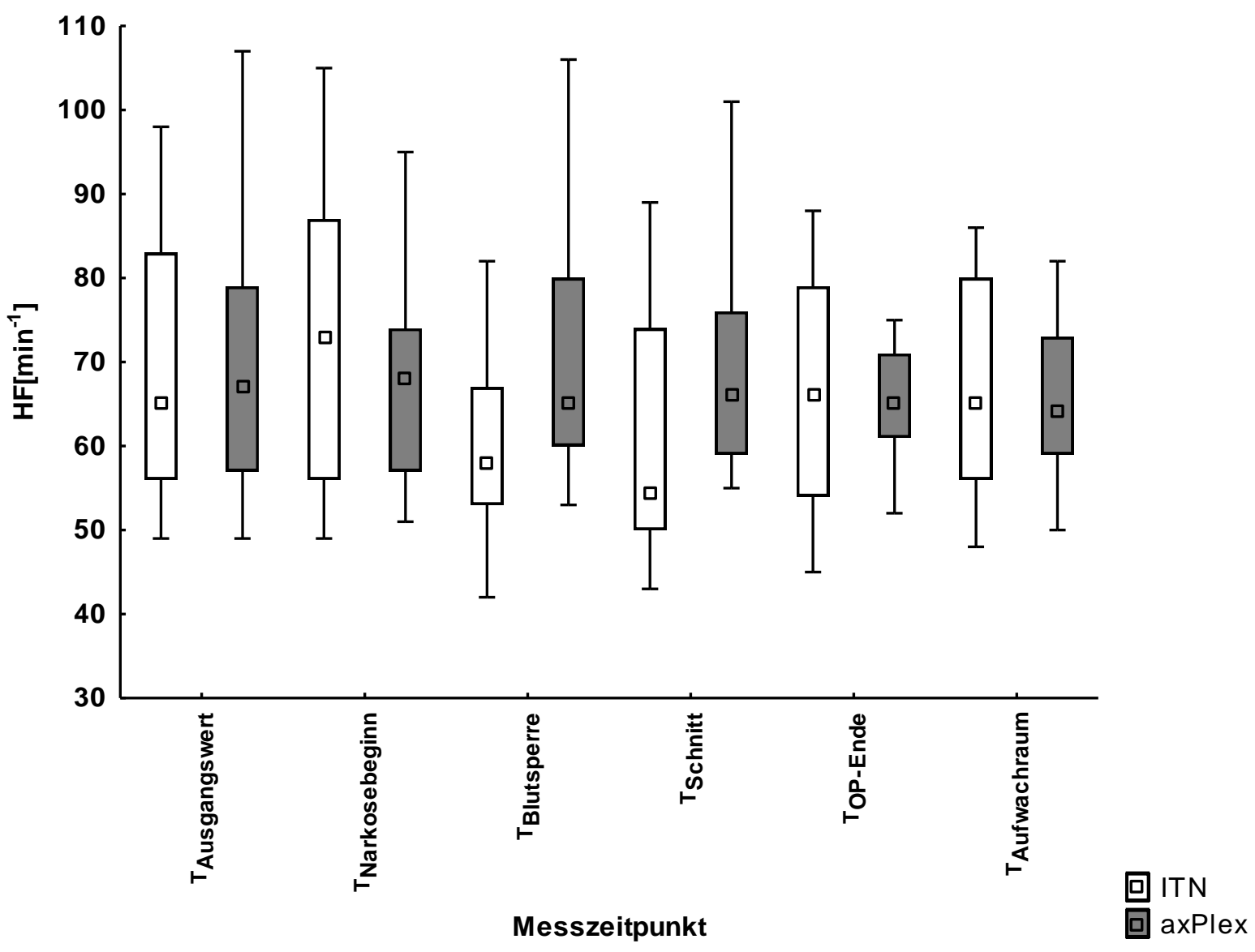

Abbildung 4: zeitlicher Verlauf der Veränderungen der Herzfrequenz in beiden Gruppen über die gesamte Messung 


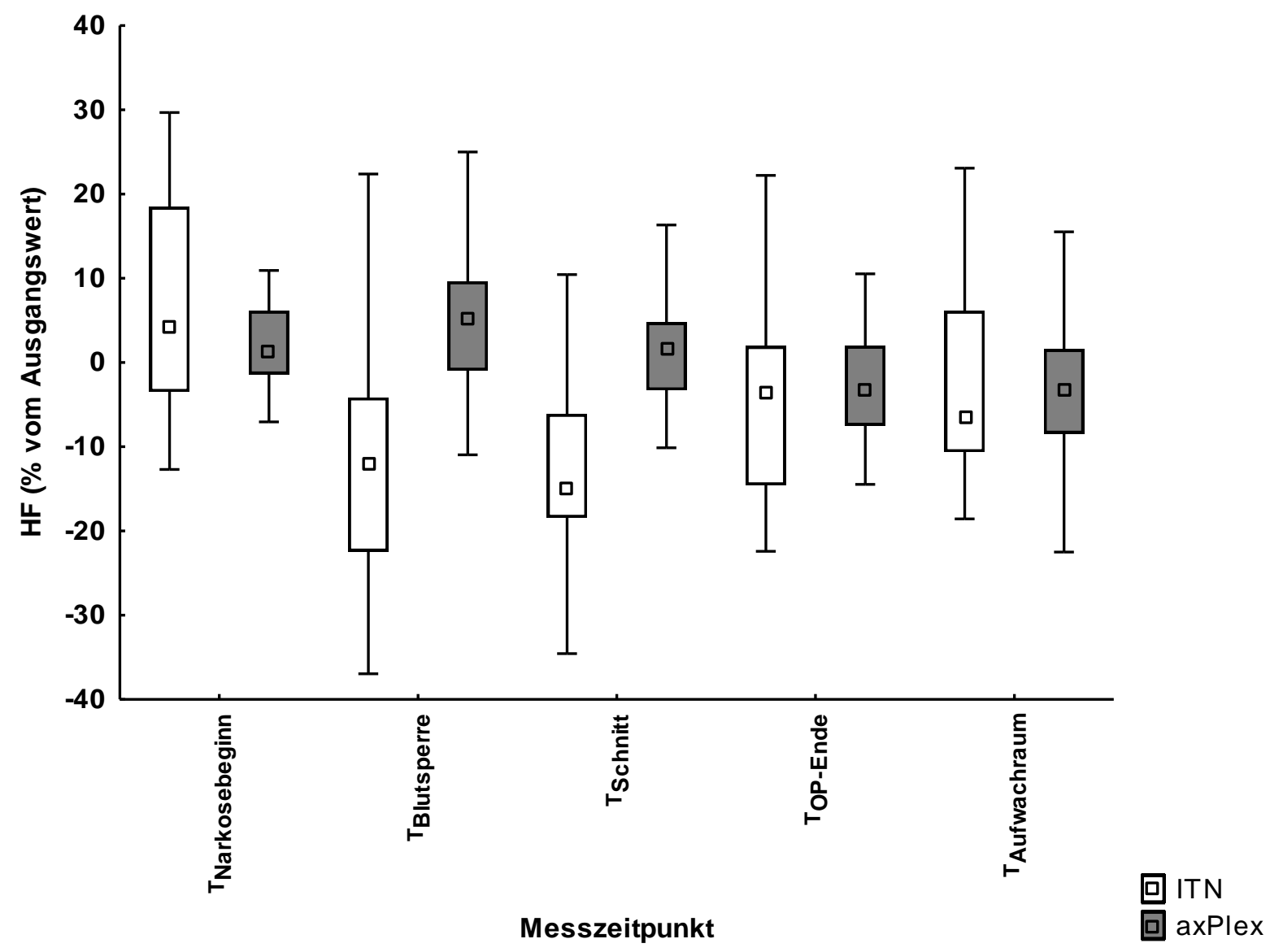

Abbildung 5: zeitlicher Verlauf der prozentualen Änderung der Herzfrequenz in beiden Gruppen über die gesamte Messung 


\subsection{Mittlerer arterieller Druck}

Während der einzelnen Messzeitpunkte wurden der systolische $\left(\mathrm{RR}_{\text {syst }}\right)$ und der diastolische $\left(\mathrm{RR}_{\text {diast }}\right)$ Blutdruck in $\mathrm{mmHg}$ erfasst. Zur statistischen Auswertung erfolgte aus den gemessenen Werten die Berechnung des mittleren arteriellen Drucks (MAD) anhand folgender Formel:

$$
\mathrm{MAD}=\mathrm{RR}_{\text {diast }}+{ }^{1 / 3} \times\left(\mathrm{RR}_{\text {syst }}-\mathrm{RR}_{\text {diast }}\right)
$$

Wie bei allen Parametern, so wurde auch beim MAD ein Ausgangswert zum Messzeitpunkt $\mathrm{T}_{\text {Ausgangswert }}$ bestimmt. In der ITN-Gruppe lagen die Werte des MAD zwischen 67 und $111 \mathrm{mmHg}$ (Median = $90 \mathrm{mmHg}$ ) und in der axPlex-Gruppe zwischen 74 und $127 \mathrm{mmHg}$ (Median $=93 \mathrm{mmHg})$. Es zeigte sich kein signifikanter Unterschied zwischen beiden Untersuchungsgruppen $(p=0,19)$. Im folgenden Messzeitpunkt $T_{\text {Narkosebeginn }}$ konnte ein signifikanter Unterschied beider Gruppen in der prozentualen Abweichung vom Ausgangswert nachgewiesen werden $(p=0,0001)$. Die MAD-Werte wichen in der ITNGruppe stärker vom Ausgangswert ab als in der axPlex-Gruppe (ITN $-10-+36 \%$ Median $=+12 \%$; axPlex $-11-+8 \%$ Median $=+2 \%$ ). Bei den absoluten Werten war hingegen kein signifikanter Unterschied zwischen beiden Gruppen nachweisbar ( $p=0,25)$. Im weiteren Verlauf der Messung zeigten sich zum Zeitpunkt $\mathrm{T}_{\text {Blutsperre }}$ signifikante Unterschiede sowohl bei den absoluten Werten (ITN 55-107 mmHg, Median = $71 \mathrm{mmHg}$; axPlex 83-126 mmHg, Median $=104 \mathrm{mmHg} ; \quad \mathrm{p}=0,000000)$ als auch bei den prozentualen Abweichungen $($ ITN $-50-+13 \%$, Median $=-19 \%$, axPlex $-6-+31 \%$, Median $=+11 \% ; p<0,001)$. Die größere Differenz zum Ausgangswert lag auf Seiten der ITN-Gruppe. Diese Ergebnisse bestätigten sich auch bei der Auswertung der folgenden Messpunkte. Sowohl bei den absoluten Werten $\left(\mathrm{T}_{\text {Schnitt }} \mathrm{p}=0,000000 ; \mathrm{T}_{\text {OP-Ende }} \mathrm{p}=0,0002\right)$ als auch bei den prozentualen Abweichungen $\left(T_{\text {Schnitt }} \mathrm{p}<0,001 ; \mathrm{T}_{\text {OP-Ende }} \mathrm{p}=0,006\right)$ ergab sich jeweils eine signifikant größere Differenz vom Ausgangswert auf Seiten der ITN-Gruppe. Zum Zeitpunkt $\mathrm{T}_{\text {Aufwachraum }}$ waren wiederum keine signifikanten Unterschiede mehr nachweisbar. Die absoluten Werte des MAD lagen in der ITN-Gruppe zwischen 72 und $108 \mathrm{mmHg}$ (Median =90,5 mmHg) und in der axPlex-Gruppe zwischen 73 und $115 \mathrm{mmHg}$ (Median $=94 \mathrm{mmHg}$ ) $\mathrm{p}=0,15$. In der ITN-Gruppe zeigten sich prozentuale Abweichungen zwischen $-22 \%$ und $+25 \%$ $($ Median $=-7 \%)$ und in der axPlex-Gruppe zwischen $-10 \%$ und $+20 \% \quad($ Median $=+3 \%)$ $\mathrm{p}=0$,76. In den Abbildungen 6 und 7 ist der zeitliche Verlauf der absoluten Werte des 
mittleren arteriellen Drucks und der prozentualen Abweichungen vom Ausgangswert beider Gruppen über die gesamte Messdauer dargestellt.

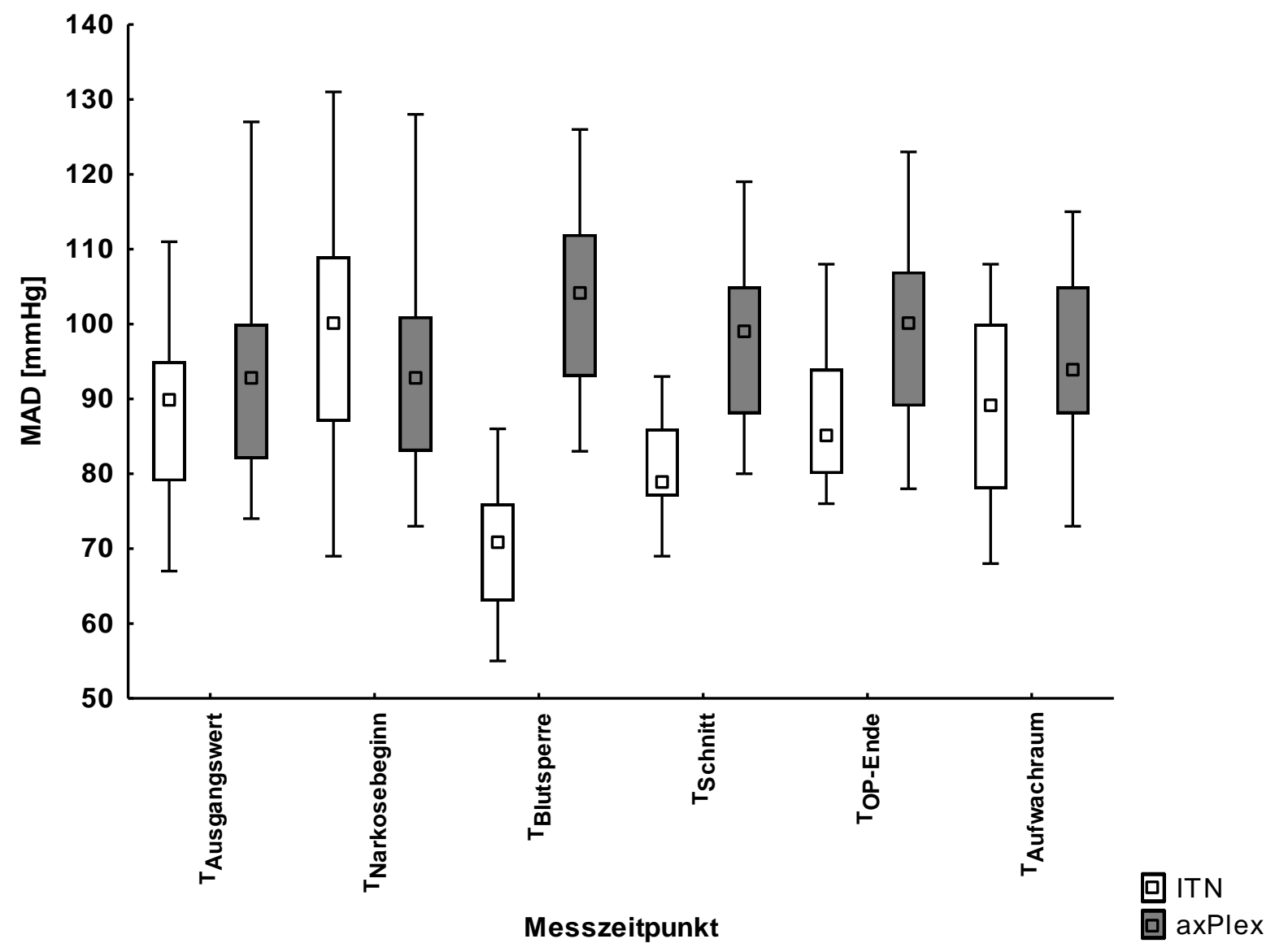

Abbildung 6: zeitlicher Verlauf der absoluten Werte des MAD in beiden Gruppen über die gesamte Messung 


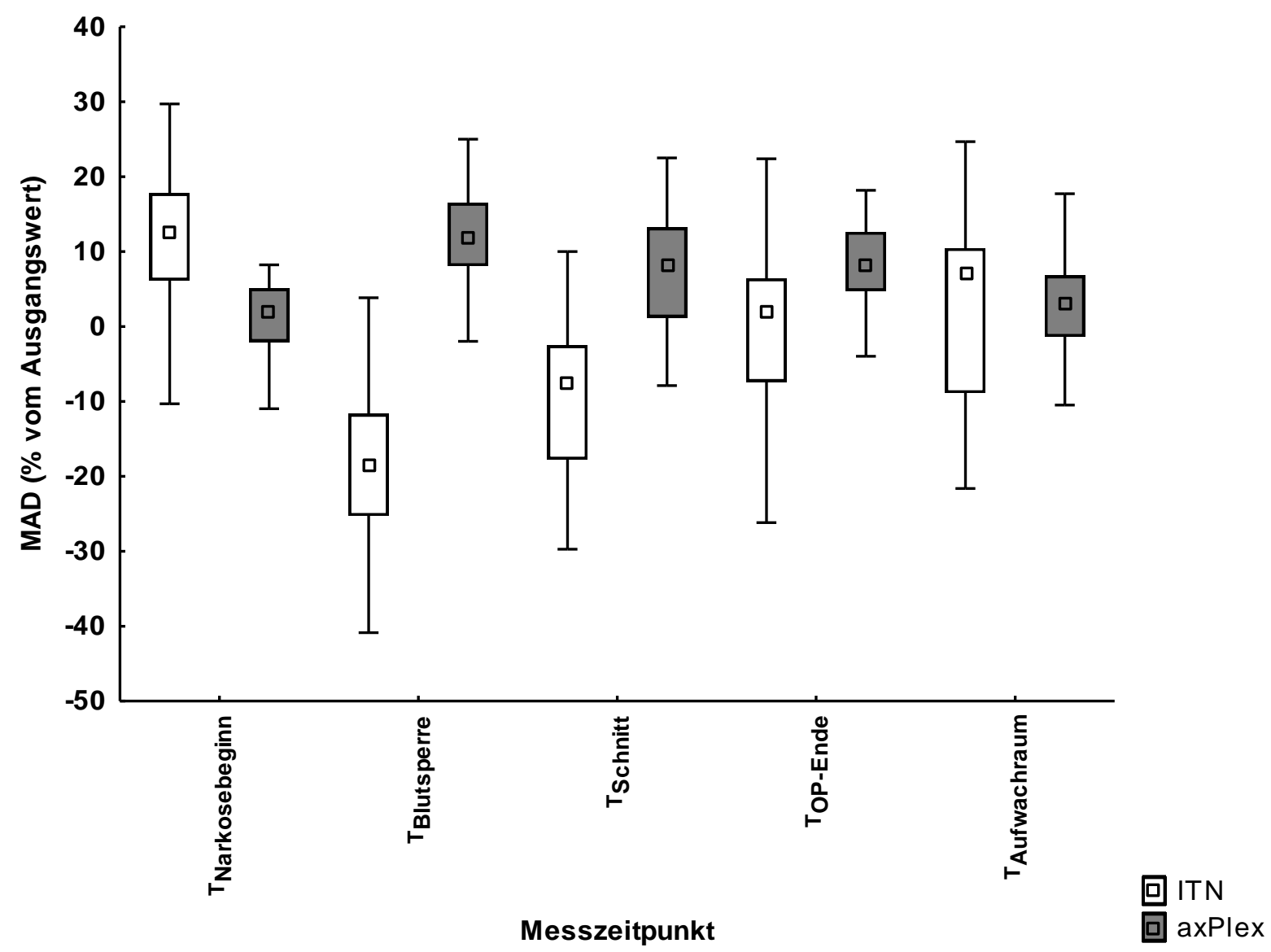

Abbildung 7: zeitlicher Verlauf der prozentualen Änderung des MAD in beiden Gruppen über die gesamte Messung 


\subsection{Elektrosympathikographie}

Die Hautwiderstandsmessung erfolgte an der nicht zu operierenden Hand. Die Auswertung der Ergebnisse der Hautwiderstandsmessung zeigte bereits bei den ermittelten Ausgangswerten zum Messzeitpunkt $\mathrm{T}_{\text {Ausgangswert }}$ einen signifikanten Unterschied zwischen beiden Gruppen $(p=0,02)$. In der ITN-Gruppe lagen die Werte zwischen 75 und 746 kOhm $($ Median $=281 \mathrm{kOhm})$ und in der axPlex-Gruppe zwischen 66 und $392 \mathrm{kOhm}$ $($ Median $=180 \mathrm{kOhm})$. Der Messzeitpunkt $\mathrm{T}_{\text {Narkosebeginn }}$ zeigte hingegen keinen signifikanten Unterschied bei den absoluten Werten $(p=0,91)$, jedoch ergab die Auswertung einen signifikanten Unterschied bei den prozentualen Abweichungen vom Ausgangswert beider Gruppen $(p=0,0001)$. Die absoluten Werte lagen in der ITN-Gruppe zwischen 58 und $470 \mathrm{kOhm}($ Median $=122 \mathrm{kOhm})$ und in der axPlex-Gruppe zwischen 50 und $543 \mathrm{kOhm}$ $($ Median $=143 \mathrm{kOhm})$. Dabei wichen die Werte in der ITN-Gruppe signifikant stärker vom Ausgangswert ab (ITN -80- +9\%, Median=-52\%) als in der axPlex-Gruppe $($ axPlex $-45-+40 \%$, Median $=-24 \%)$. Bei den nachfolgenden Messzeitpunkten waren bei den absoluten Werten $\left(\mathrm{T}_{\text {Blutsperre }} \mathrm{p}=0,000001, \mathrm{~T}_{\text {Schnitt }} \mathrm{p}=0,000000, \mathrm{~T}_{\text {Op-Ende }} \mathrm{p}=0,000000\right.$, $\left.\mathrm{T}_{\text {Aufwachraum }} \mathrm{p}=0,001\right)$ und den prozentualen Abweichungen $\left(\mathrm{T}_{\text {Blutsperre }} \mathrm{p}<0,001\right.$; $\left.\mathrm{T}_{\text {Schnitt }} \mathrm{p}<0,001 ; \mathrm{T}_{\text {Op-Ende }} \mathrm{p}<0,001 ; \mathrm{T}_{\text {Aufwachraum }} \mathrm{p}=0,005\right)$ signifikante Unterschiede

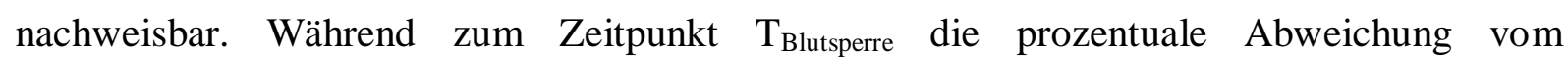
Ausgangswert in der axPlex-Gruppe signifikant größer war (ITN Median $=+14 \%$, axPlex Median $=-41 \%$ ), war diese Abweichung in den beiden folgenden Messzeitpunkten in der ITN-Gruppe größer $\left(\mathrm{T}_{\text {Schnitt }}\right.$ ITN Median $=+36 \%$, axPlex Median $=-34 \% ; \mathrm{T}_{\text {Op-Ende }}$ ITN Median $=+38 \%$, axPlex Median $=-32 \%$ ). $\mathrm{T}_{\text {Aufwachraum zeigte hingegen wieder eine größere }}$ Abweichung auf Seiten der axPlex-Gruppe (ITN Median = -2\%, axPlex Median $=-32 \%$ ) Der

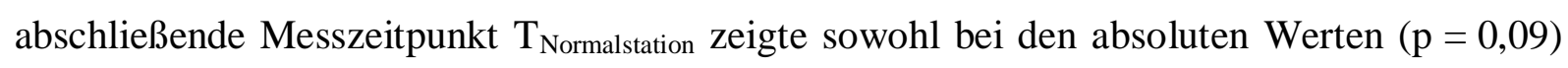
als auch bei den prozentualen Veränderungen $(\mathrm{p}=0,81)$ keine signifikanten Unterschiede zwischen beiden Gruppen. In den Abbildungen 8 und 9 ist der zeitliche Verlauf der gemessenen und prozentualen Veränderungen der Hautwiderstandswerte in beiden Gruppen dargestellt. 


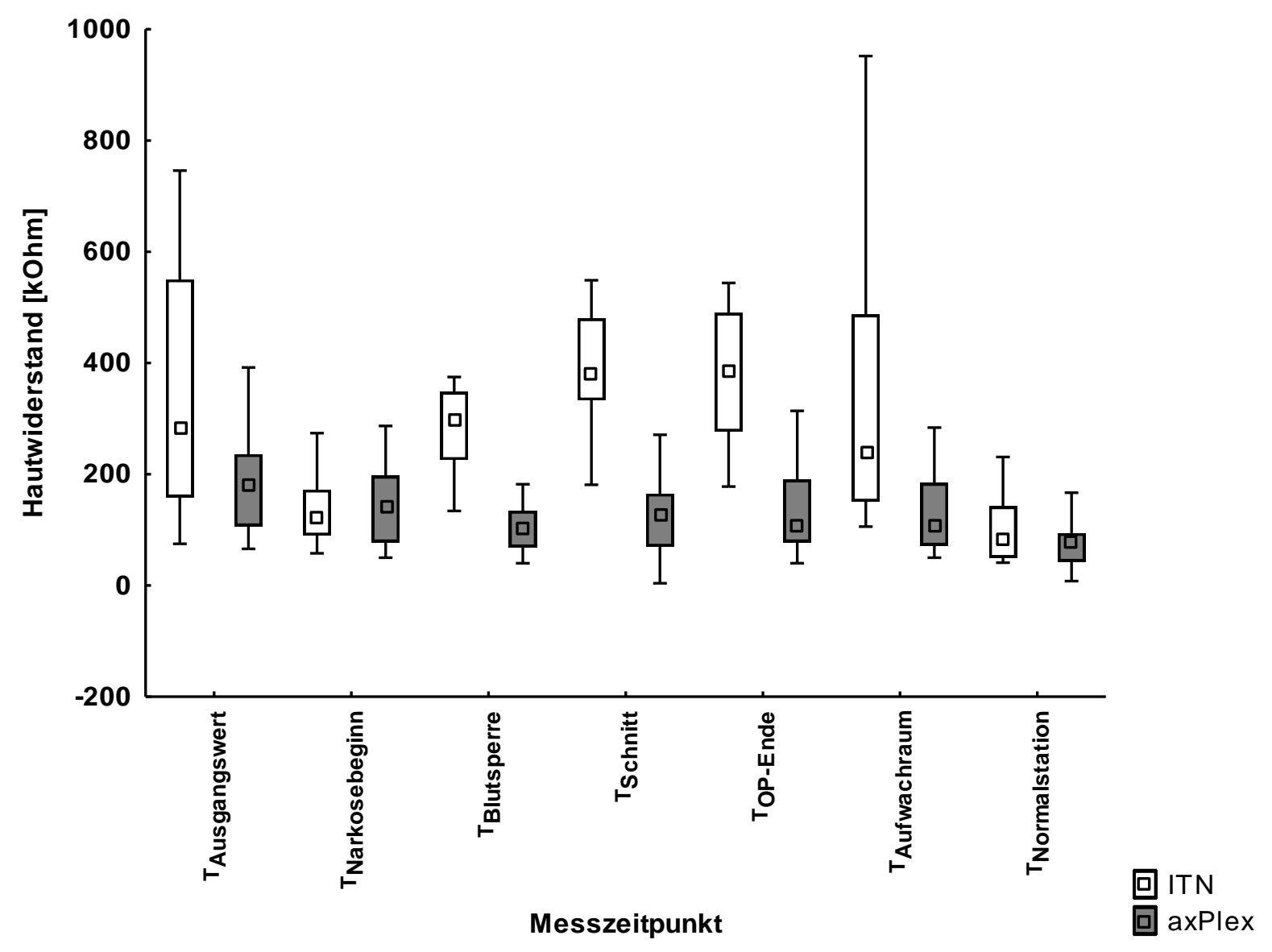

Abbildung 8: zeitlicher Verlauf der Hautwiderstandswerte in beiden Gruppen über die gesamte Messung 


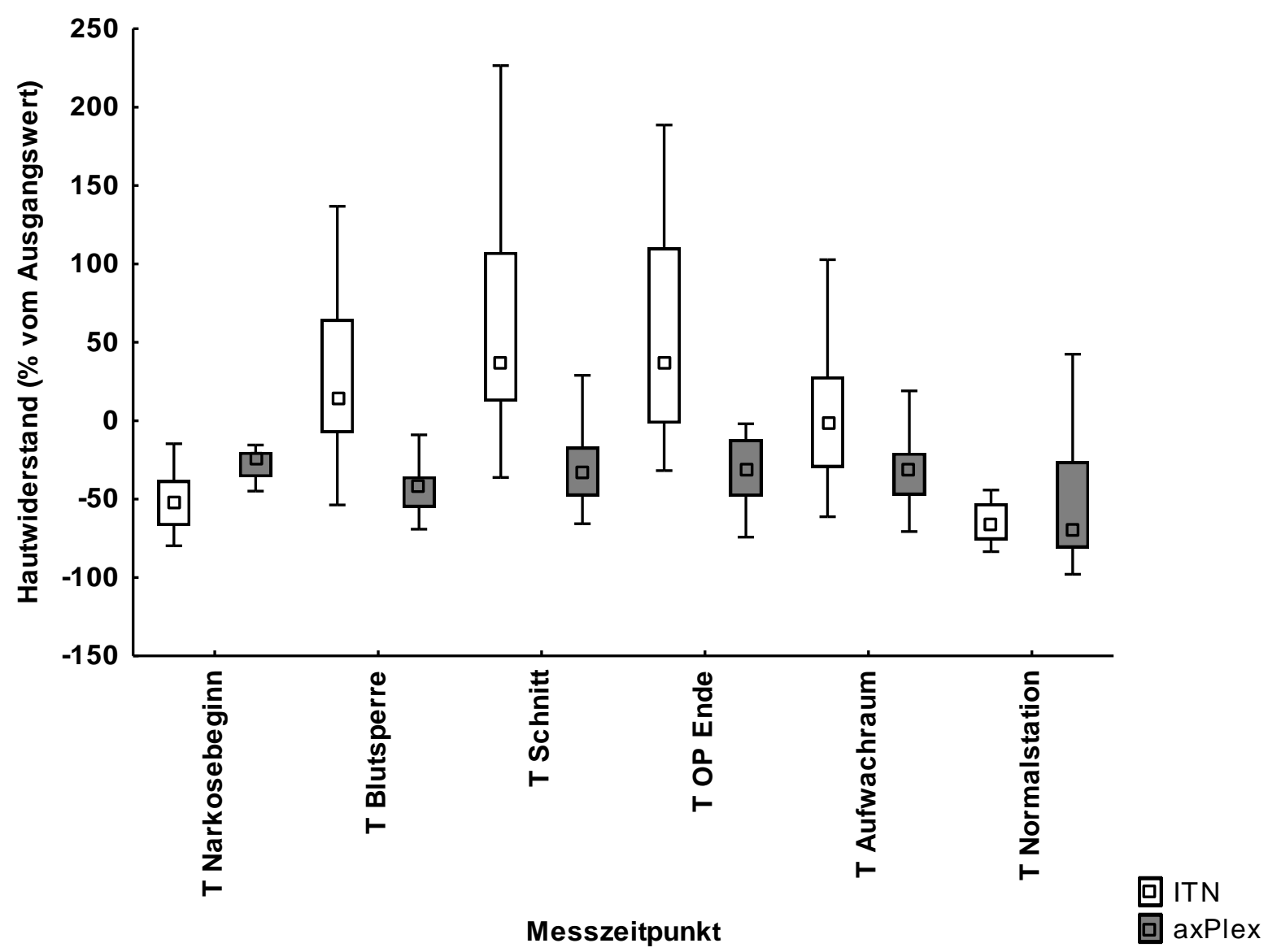

Abbildung 9: zeitlicher Verlauf der prozentualen Änderung des Hautwiderstands in beiden Gruppen über die gesamte Messung 


\subsection{Temperatur}

Die Messung der Hauttemperatur erfolgte ebenso wie die ESG-Messung an der Hand, die nicht operiert wurde. In der statistischen Auswertung wurden die absoluten Temperaturen untersucht und die Veränderungen der Hauttemperatur im Vergleich zum Ausgangswert im Verlauf der Messung. Sowohl die absoluten Werte als auch die Veränderungen wurden in Grad Celsius $\left[{ }^{\circ} \mathrm{C}\right]$ angegeben. Der zum Messzeitpunkt $\mathrm{T}_{\text {Ausgangswert }}$ bestimmte Ausgangswert zeigte keinen signifikanten Temperaturunterschied zwischen beiden Untersuchungsgruppen $(\mathrm{p}=0,72)$. In der ITN-Gruppe lagen die Werte zwischen $27,8{ }^{\circ} \mathrm{C}$ und $35,3{ }^{\circ} \mathrm{C}$ (Median $=31,3{ }^{\circ} \mathrm{C}$ ) und in der axPlex-Gruppe zwischen $28,0{ }^{\circ} \mathrm{C}$ und $35,2{ }^{\circ} \mathrm{C}$ $\left(\right.$ Median $\left.=31,5^{\circ} \mathrm{C}\right)$. Zum Messzeitpunkt $\mathrm{T}_{\text {Narkosebeginn }}$ zeigte sich ebenfalls kein signifikanter Unterschied zwischen beiden Gruppen im Bezug auf die absolute Temperatur $(p=0,43)$ und die jeweilige Temperaturabweichung $(\mathrm{p}=0,19)$. Die Analyse des folgenden Messzeitpunktes

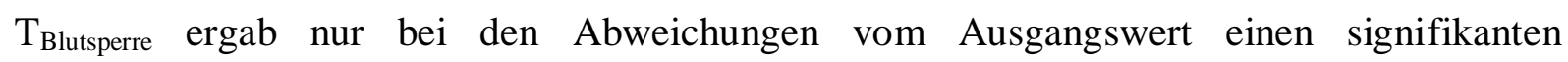
Unterschied zwischen beiden Gruppen (ITN $-11^{\circ} \mathrm{C}-\quad+2{ }^{\circ} \mathrm{C}$, Median $=-1,4{ }^{\circ} \mathrm{C}$; axPlex $-5,3{ }^{\circ} \mathrm{C}-+2{ }^{\circ} \mathrm{C}$, Median $=-2,3{ }^{\circ} \mathrm{C} ; \mathrm{p}=0,03$ ) mit einer größeren Temperaturdifferenz in der axPlex-Gruppe. Die absoluten Werte zeigten keinen signifikanten Unterschied $(\mathrm{p}=0,5)$. Die Analyse der folgenden Messzeitpunkte ergab sowohl einen signifikanten Unterschied zwischen den absolut gemessenen Temperaturen $\left(T_{\text {Schnitt }} p=0,005\right.$, $\left.\mathrm{T}_{\text {Op-Ende }} \mathrm{p}=0,001, \mathrm{~T}_{\text {Aufwachraum }} \mathrm{p}=0,024\right)$ als auch eine signifikant größere Differenz zum Ausgangswert in der axPlex-Gruppe als in der ITN-Gruppe $\left(T_{\text {Schnitt }} p<0,001\right.$, $\left.\mathrm{T}_{\text {Op-Ende }} \mathrm{p}<0,001, \mathrm{~T}_{\text {Aufwachraum }} \mathrm{p}=0,001\right)$. Die Abweichungen zum Zeitpunkt $\mathrm{T}_{\text {Schnitt }}$ lagen in der ITN-Gruppe zwischen $-1,4{ }^{\circ} \mathrm{C}$ und $+2,8{ }^{\circ} \mathrm{C}\left(\right.$ Median $\left.=+0,1^{\circ} \mathrm{C}\right)$ und in der axPlex-Gruppe zwischen $-5,6^{\circ} \mathrm{C}$ und $+4,7^{\circ} \mathrm{C}$ (Median $=-2,5^{\circ} \mathrm{C}$ ). $\mathrm{T}_{\mathrm{OP}}$ Ende zeigte Temperaturveränderungen in der ITN-Gruppe von $-3,5{ }^{\circ} \mathrm{C}$ bis $+2,8{ }^{\circ} \mathrm{C}$ (Median $=0{ }^{\circ} \mathrm{C}$ ) und in der axPlex-Gruppe von $-6,3{ }^{\circ} \mathrm{C}$ bis $+4,3{ }^{\circ} \mathrm{C} \quad\left(\right.$ Median $\left.=-3,8^{\circ} \mathrm{C}\right)$. Die Temperaturdifferenzen zum Zeitpunkt

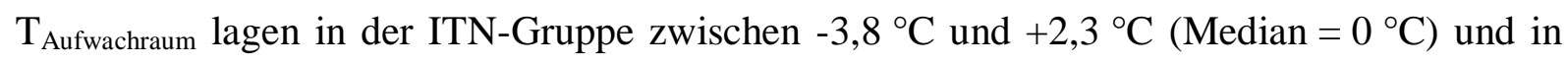
der axPlex-Gruppe zwischen $-6,2{ }^{\circ} \mathrm{C}$ und $+3,7{ }^{\circ} \mathrm{C} \quad\left(\right.$ Median $=-2,7^{\circ} \mathrm{C}$ ). Der letzte

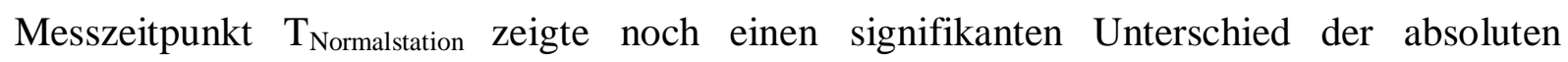
Temperaturwerte (ITN $28,4{ }^{\circ} \mathrm{C}-34,5^{\circ} \mathrm{C}, \quad$ Median $=32,7^{\circ} \mathrm{C}$; $\quad$ axPlex $\quad 32,1^{\circ} \mathrm{C}-36{ }^{\circ} \mathrm{C}$, Median $\left.=34,35{ }^{\circ} \mathrm{C} ; \mathrm{p}=0,001\right)$. Es ergab sich aber kein signifikanter Unterschied mehr zwischen den Temperaturabweichungen der beiden Gruppen (ITN $-2{ }^{\circ} \mathrm{C}-\quad+4,9{ }^{\circ} \mathrm{C}$, Median $=+1,9^{\circ} \mathrm{C}$; axPlex $0{ }^{\circ} \mathrm{C}-+5^{\circ} \mathrm{C}$, Median $=+1,8^{\circ} \mathrm{C} ; \mathrm{p}=0,52$ ) In Abbildung 10 und 11 
ist der Verlauf der gemessenen Werte der Hauttemperatur und der Abweichungen vom Ausgangswert in beiden Gruppen dargestellt.

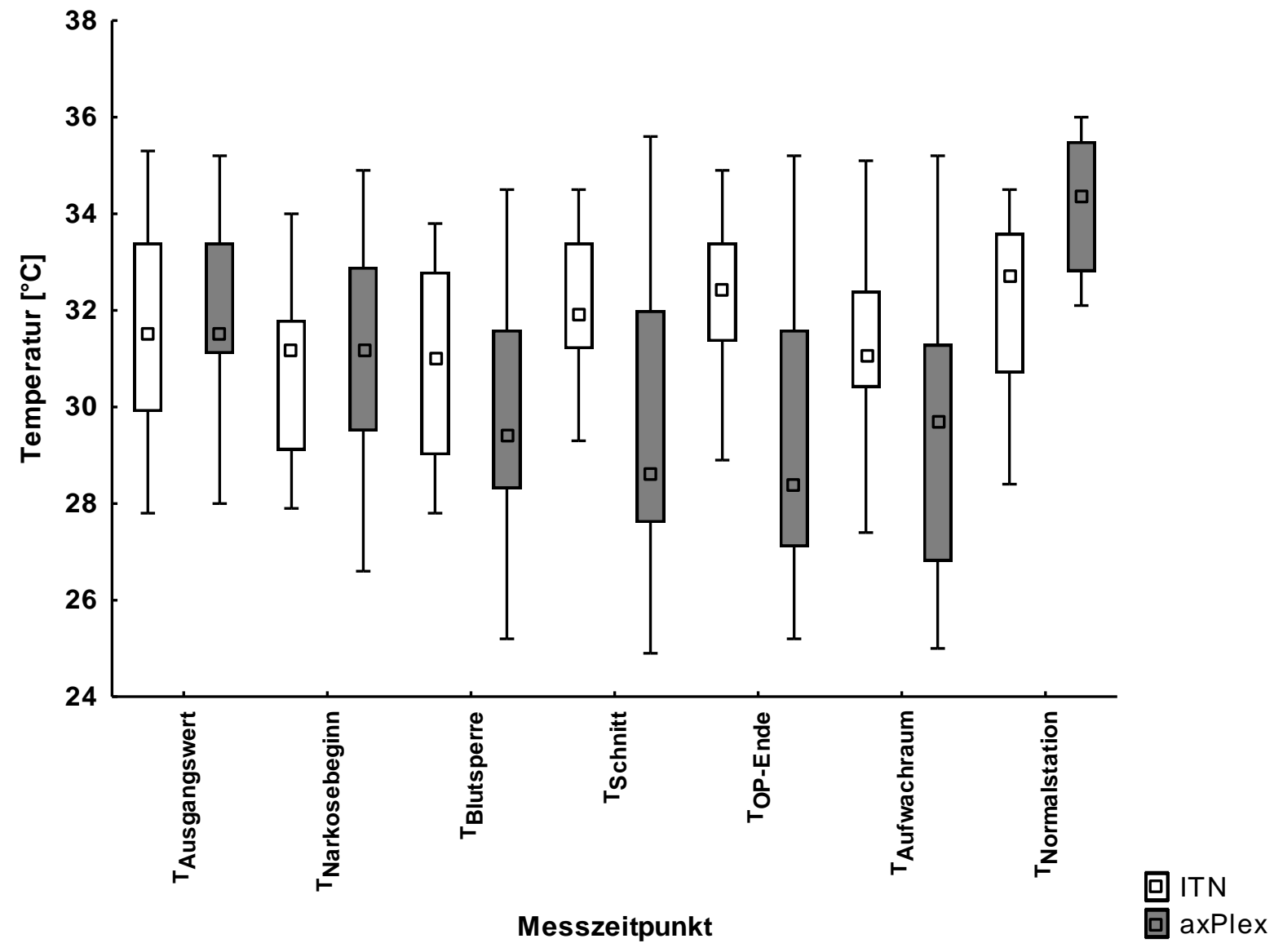

Abbildung 10: zeitlicher Verlauf der Hauttemperatur in beiden Gruppen über die gesamte Messung 


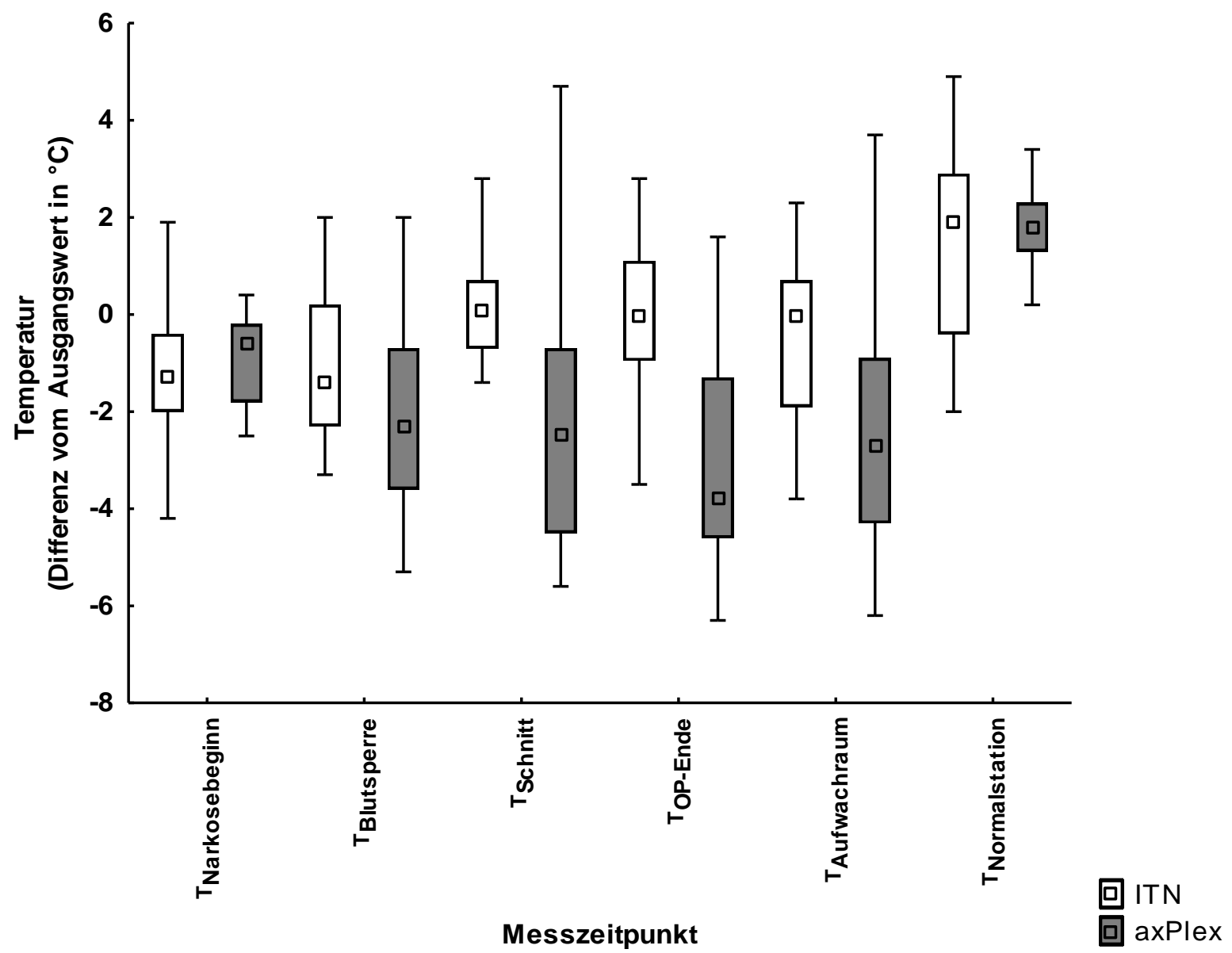

Abbildung 11: zeitlicher Verlauf der Abweichung der Hauttemperatur vom Ausgangswert in beiden Gruppen 


\section{Diskussion}

In der Anästhesie stehen zur optimalen operativen Versorgung des Patienten je nach Art des notwendigen operativen Eingriffs verschiedenste Narkoseverfahren zur Verfügung. Aus den vielfältigen Möglichkeiten der Allgemein- und der Regionalanästhesie muss unter Berücksichtigung aller wichtigen Einflussfaktoren wie beispielsweise Alter und Vorerkrankungen des Patienten das individuell optimale Verfahren für die jeweilige Operation bei einem Patienten ausgewählt werden. Regionale Verfahren bieten gerade bei der zunehmenden Zahl von ambulanten Operationen, als auch bei der steigenden Zahl von alten und schwerkranken Patienten, die einer Operation bedürfen, eine gute und sichere Alternative zur Allgemeinanästhesie. Ferner bieten die regionalen Verfahren eine ganze Reihe von Möglichkeiten, um dem Patienten eine adäquate perioperative Schmerztherapie zukommen zu lassen. Da die negativen Auswirkungen einer überschießenden perioperativen Stressreaktion und ihre Folgen für das Outcome des Patienten - wie oben beschrieben - immer besser bekannt geworden sind, ist es von großem Interesse, den Patienten bestmöglich vor diesen Folgen zu schützen. Beim Vergleich von Regionalanästhesie und Allgemeinanästhesie im Bezug auf die Freisetzung von Stresshormonen, vor allem aus der Nebenniere, zeigt sich, dass es unter Regionalanästhesie zu einer geringeren Hormonausschüttung kommt. Die Stressreaktion ist herabgesetzt (Wacker et al. 2005). Adams et al. verglichen die Hormonspiegel von Patientinnen, die eine Sectio unter Allgemeinanästhesie erhielten, mit denen von Patientinnen, bei denen die Narkose mittels Periduralkatheter erfolgte. Die Ausschüttung von Katecholaminen, von ACTH und Kortisol waren in der Allgemeinanästhesiegruppe wesentlich höher. Als Ursache sahen die Forscher die durch die Periduralanästhesie bedingte Sympathikolyse an (Adams et al. 1989). Weitere Untersuchungen konnten diese Ergebnisse auch während anderer Operationen bestätigen (Celic-Spuzic 2011, Wolf 2012). Die Verfahren, die zur Allgemeinanästhesie zur Verfügung stehen, unterscheiden sich auch untereinander im Bezug auf die hormonale Stressantwort. So schirmen total intravenöse Anästhesien (TIVA) mit Propofol die Hormonausschüttung durch intraoperativen Stress besser ab als inhalative Anästhesien mit Sevofluran (Winterhalter et al. 2007b). Ob bei einer TIVA Remifentanil oder Sufentanil zur Analgesie verwendet wird hat im Bezug auf die stressabschirmende Wirkung keinen Einfluss (Brockmann et al. 2000).

Für größere Operationen kommen häufig Spinalanästhesien oder Epiduralanästhesien zur Anwendung. Obschon diese Verfahren Vorteile gegenüber der Allgemeinanästhesie bieten, so kann es gerade bei Spinalanästhesien durch die ausgeprägte Sympathikolyse zu erheblichen 
kardialen Nebenwirkungen kommen, die mit der Ausbreitungshöhe zunehmen. Eine Ausbreitung über das Segment TH1 hinaus kann mit massiven Blutdruckabfällen und Bradykardien bis zu Herzstillständen einhergehen. Die zeitlich limitierte Wirkung schränkt die Anwendung der Spinalanästhesie weiterhin ein (Wacker et al. 2005). Im Vergleich bietet die Epiduralanästhesie für große chirurgische Eingriffe eine größere hämodynamische Stabilität bei ausreichender peri- und postoperativer Analgesie. Obwohl die Sympathikolyse der Epiduralanästhesie geringer ist, führt eine Kombination aus Allgemeinanästhesie und Epiduralanästhesie im Vergleich zur alleinigen Allgemeinanästhesie zu einer deutlichen Reduktion der humoralen Stressantwort (Ahlers et al. 2008, Kawagoe et al. 2011). Daraus lässt sich schließen, dass die multimodale Anästhesie aus Allgemeinanästhesie und epiduraler Schmerztherapie zurzeit das Mittel der Wahl ist, um den Patienten, bei entsprechender OpIndikation, peri- und postoperativ ideal zu versorgen, ihn vor Schmerzen zu bewahren und die Folgen der hormonalen Stressantwort abzumildern. Im Unterschied zur Allgemeinanästhesie kann bei wachen Patienten der Einfluss äußerer Stressoren und die bestehende emotionale Situation Einfluss auf die Ausprägung der Stressreaktion haben. Eine generelle Aussage, dass eine Regionalanästhesie für Patienten stressreduzierter verläuft als eine Allgemeinanästhesie, ist somit niemals vorhersagbar. Nur eine Erfassung empfindlicher Stressindikatoren spiegelt das direkte nervale Impulsmuster auf intraoperative Stressreize wieder.

Im Fokus dieser Studie stand der Einfluss des Anästhesieverfahrens bei handchirurgischen Operationen auf die Sympathikusaktivität, mit der Fragestellung, ob eine Regionalanästhesie effektiver einer überschießenden Stressantwort vorbeugt als eine Allgemeinanästhesie. Die Erfassung der Stressantwort erfolgte durch die perioperative Messung non-invasiver Parameter wie Herzfrequenz, mittlerem arteriellem Druck, Hautwiderstand und Hauttemperatur. Die Narkosetiefe wurde mittels BIS kontrolliert, um Fälle mit großer Abweichung bzw. mit zu flacher oder zu tiefer Anästhesie auszuschließen. Ferner erfolgte eine Analyse des peri- und postoperativen Schmerzempfindens über eine numerische Ratingskala (NRS), sowie die Erfassung des intra- und postoperativen Schmerzmittelbedarfs der Patienten.

Zum besseren Vergleich wurde die gesamte Messung in sieben Messzeitpunkte $\left(\mathrm{T}_{\text {Ausgangswert }}\right.$, $\mathrm{T}_{\text {Narkosebeginn }}, \mathrm{T}_{\text {Blutsperre, }}, \mathrm{T}_{\text {Schnitt, }}, \mathrm{T}_{\mathrm{OP}-\text { Ende }}, \mathrm{T}_{\text {Aufwachraum, }} \mathrm{T}_{\text {Normalstation }}$ ) unterteilt. Bei der Analyse der $\mathrm{zu}$ Beginn in $\mathrm{T}_{\text {Ausgangswert }}$ erhobenen Ausgangswerte wurde ein signifikanter Unterschied zwischen den ESG-Werten beider Untersuchungsgruppen festgestellt. Es fanden sich signifikant höhere Werte in der ITN-Gruppe. Die Werte der anderen ermittelten Parameter 
ergaben keine signifikanten Unterschiede. Diese Beobachtung deckt sich mit den Ergebnissen, die bereits in Studien von Winterhalter und Zaps erfasst wurden, wonach bei den Hautwiderstandswerten eine große intra- und interindividuelle Breite vorliegt (Winterhalter et al. 2007b, Zaps 2010).

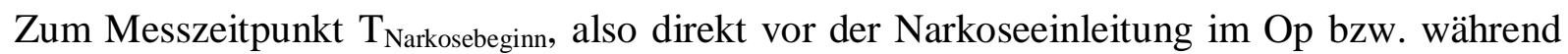
der Plexusanlage, wiesen beide Gruppen eine Steigerung des MAD und einen Abfall des Hautwiderstandes im Sinne eines Einleitungsstresses auf (Janitzki 2010a). Auch wenn sich bei beiden Parametern die absoluten Werte nicht signifikant unterschieden, so wiesen doch die prozentualen Veränderungen vom Ausgangswert auf einen höheren Stresspegel in der ITN-Gruppe hin, in der zudem die Herzfrequenz im Median von 65 Schlägen pro Minute auf 73 Schläge pro Minute anstieg. Dies könnte ein Hinweis darauf sein, dass die Anlage einer Regionalanästhesie, bei der der Patient wach ist, weniger Stress auslöst als die unmittelbar bevorstehende Allgemeinanästhesie, die mit einem Bewusstseins- und Kontrollverlust einhergeht.

Im weiteren Messverlauf ( $\mathrm{T}_{\text {Blutsperre}}$ ) konnten in der ITN-Gruppe die erwarteten, narkoseinduzierten Abfälle der Kreislaufparameter beobachtet werden. Der parallel dazu ansteigende Wert des Hautwiderstandes untermauert die Beobachtung einer reduzierten, in dieser Phase gedämpften Sympathikusaktivität. Die gemessenen BIS-Werte, die eine ausreichende Narkosetiefe belegten, sowie die Tatsache, dass der Abfall der Kreislaufparameter keine kritischen Werte erreichte, sprechen für die Qualität der durchgeführten Allgemeinanästhesie. Das Wirkprofil der benutzten Anästhetika mit Dämpfung des cerebralen und neurovegetativen Funktionszustandes, und damit der Kreislaufregelmechanismen, lässt diese Beeinflussung der Kreislaufparameter auch erwarten. Im Gegensatz dazu zeigte sich in der axPlex-Gruppe ein Anstieg des MAD sowie ein Abfall des Hautwiderstandes im Vergleich zum vorangehenden Messzeitpunkt. Unterschiedlichste Stressoren könnten für diese Veränderungen verantwortlich sein. Zum einen die ungewohnte Umgebung des Operationssaals, sowie die Unsicherheit, ob trotz durchgeführter Regionalanästhesie möglicherweise intraoperativ Schmerzen oder andere unangenehme Ereignisse zu erwarten sind.

Bei der Beobachtung der Hauttemperatur fiel auf, dass es in der axPlex-Gruppe zu einem signifikanten Temperaturabfall im Vergleich zur ITN-Gruppe kam. Vermutlich bedingten die in der Allgemeinanästhesie verwendeten Medikamente durch ihre vasodilatierende Wirkung die konstantere Hauttemperatur in der ITN-Gruppe. Eine weitere Ursache für den Temperaturunterschied könnte darin begründet sein, dass in der Gruppe der 
Allgemeinanästhesie besser auf den Wärmeerhalt geachtet wurde als in der Gruppe der Regionalanästhesie, um Kältezittern und ein Auskühlen der Patienten zu verhindern. Interessanterweise klagte keiner der Patienten unter Regionalanästhesie über ein Kältegefühl. Auch auf gezielte Nachfrage wurde diese Missempfindung verneint.

Zum Messzeitpunkt $\mathrm{T}_{\text {Schnitt }}$ wurden die stabilen Kreislaufparameter bestätigt. Der Anstieg des MAD und der Abfall der Herzfrequenz waren moderat. Der Operationsbeginn ging nicht mit einem Anstieg des Stresslevels einher. Die Narkosetiefe und die Analgesie können deshalb als optimal bewertet werden. Der bereits zuvor beobachtete Temperaturabfall in der axPlexGruppe setzte sich fort, dies scheint die Theorie der Auskühlung zu untermauern.

Mit dem Ende der OP ( $\left.\mathrm{T}_{\mathrm{OP}-\mathrm{Ende}}\right)$ kam es aufgrund der nachlassenden Anästhetikawirkung zu einem Anstieg des MAD und der Herzfrequenz auf Seiten der ITN-Gruppe, wobei die Ausgangswerte wieder nahezu erreicht wurden. Ähnlich sah es bei der axPlex-Gruppe aus. Interessanterweise fiel hier der Hautwiderstandswert nochmals ab, was möglicherweise durch die Freude der überstandenen OP verursacht sein kann. Der weiterhin hohe ESG-Wert der ITN-Gruppe ist vermutlich durch den Überhang der Anästhetika mit noch dämpfender Wirkung auf den neurovegetativen Funktionszustand zu erklären.

Die Messergebnisse im Aufwachraum ( $\mathrm{T}_{\text {Aufwachraum }}$ ) zeigten stabile Kreislaufverhältnisse in beiden Gruppen, die nahezu den Ausgangswerten entsprachen. Die Auswertung der ESGWerte ergab jedoch einen signifikanten Unterschied zwischen beiden Gruppen. Hier fiel vor allem die große Standardabweichung in der ITN-Gruppe auf. Eine Ursache liegt darin begründet, dass einige Patienten nach der Allgemeinanästhesie im Aufwachraum nochmals einschliefen, dies führte zu einer Dämpfung der Sympathikusaktivität mit den entsprechend hohen ESG-Werten. Die beobachtete intraoperative Auskühlung der Patienten der axPlexGruppe war bereits im Aufwachraum rückläufig. Dieser Trend setzte sich in der postoperativen Kontrolle auf der Normalstation ( $\left.\mathrm{T}_{\text {Normalstation }}\right)$ fort.

Betrachtet man nun die Messung insgesamt, so fällt auf, dass in beiden Gruppen die Herzfrequenz über die gesamte Messung weitestgehend stabil war. Selbst bei Änderung des Blutdrucks waren keine wesentlichen Veränderungen der Herzfrequenz messbar. Eine mögliche Ursache könnte in der Verwendung von Anästhetika mit optimal ausgewogener neurovegetativer Dämpfung, gleichsam einer B-Blockade, liegen. Aber auch die Auswahl der Patienten selbst könnte hier eine entscheidende Rolle spielen. Die beobachteten moderaten Blutdruckveränderungen konnten möglicherweise bei den Patienten, die alle als herzgesund zu beurteilen sind, ohne eine wesentliche Steigerung der Herzfrequenz erreicht werden. 
In der ITN- Gruppe blieben über den gesamten intraoperativen Messverlauf Blutdrucksteigerungen und Anstiege der Herzfrequenz im Sinne einer Stressreaktion aus. Hier spielen mit hoher Wahrscheinlichkeit die BIS gesteuerte Narkosetiefe und die intraoperative Analgetikagabe eine Rolle. Gerade die regelhafte Gabe von Metamizol Natrium $1 \mathrm{H}_{2} \mathrm{O}$ (Novaminsulfon-ratiopharm®, Ratiopharm, Ulm, Deutschland) könnte durch seine hypotone Wirkung mögliche intraoperative Blutdruckanstiege verschleiert haben.

Die Beobachtung, dass weder intraoperativ noch postoperativ bis zur Entlassung einem Patienten der axPlex-Gruppe ein Analgetikum verabreicht werden musste, spricht eindeutig für die Qualität der durchgeführten Regionalanästhesie, sowie für die Auswahl der verwendeten Lokalanästhetika und deren Dosierung. Da die Patienten nach der Allgemeinanästhesie im Aufwachraum und auch auf der Normalstation signifikant höhere Schmerzen hatten, muss das postoperative Schmerzmanagement in beiden Phasen besonders beachtet werden. Die gemessenen Zeiten für den Aufenthalt im Aufwachraum zeigen, dass die Regionalanästhesie wirtschaftliche Vorteile gegenüber der Allgemeinanästhesie hat. Die Patienten der axPlex-Gruppe konnten signifikant schneller aus dem Aufwachraum verlegt werden. Dieser Vorteil hätte sich sicherlich auch in der Auswertung der Aufenthaltsdauer im Krankenhaus gezeigt, wenn die Patienten der axPlex-Gruppe nicht erst nach der Durchführung der Abschlussmessung am Ende des OP-Programms entlassen worden wären. Die Regionalanästhesie bietet somit wirtschaftliche Vorteile bei besserer Analgesie.

Die Studie konnte ferner die Möglichkeiten, aber auch die Einschränkungen der Hautwiderstandsmessung zeigen, die bisher in der Medizin im Gegensatz zur Psychologie und Philosophie noch keinen Einzug gehalten hat. Die Veränderungen des Hautwiderstandes im Verlauf der Messung der ITN-Gruppe decken sich mit den Beobachtungen anderer Studien beispielsweise von Storm et al. (Storm et al. 2002). Hier zeigte sich mit Beginn der Einleitung und Vertiefung der Narkose eine Zunahme des Hautwiderstandes, passend zur Narkosetiefe und Reduktion von Herzfrequenz und Blutdruck, mit der Einschränkung, dass zum OP-Ende zwar die Kreislaufparameter wieder die Ausgangswerte erreicht hatten, der Hautwiderstand jedoch noch relativ hoch war. Hier scheint eine Ursache darin zu liegen, dass die Messungen der Studie im OP nicht bis zum vollständigen Abklingen der Anästhetika durchgeführt werden konnten. Eine längere Messung unmittelbar nach der Operation im OP hätte zu einer größeren Verzögerung im OP-Ablauf geführt. Die hier beobachteten Veränderungen des Hautwiderstandes während der Regionalanästhesie zeigten jedoch auch, dass die 
Hautwiderstandsmessung beim wachen Patienten sehr unspezifisch ist, da gerade im wachen Zustand verschiedene Faktoren die Sympathikusaktivität beeinflussen können. Die intraoperativ erfassten Hautwiderstandsänderungen beim wachen Patienten verdeutlichen die insgesamt mannigfaltigen Reaktionen des Neurovegetativums auf mögliche Schmerzen und andere Stressoren und Umstände.

Es wäre wünschenswert gewesen, wenn während der Operation parallel auch an der zu operierenden Hand der Hautwiderstand gemessen worden wäre, um den dämpfenden Einfluss der Regionalanästhesie auf den sympathischen Signalfluss zu erfassen. Aus Gründen der Sterilität war dies jedoch nicht möglich. Die bereits erwähnten intra- und interindividuellen Unterschiede des Hautwiderstandes stellen des Weiteren ein Problem dar, das noch weiterer Forschung bedarf, um definitive quantitative Aussagen machen zu können. Die Möglichkeiten der Hauwiderstandsmessung werden in verschiedenen Studien kontrovers diskutiert. Einige Studien beispielsweise von Winterhalter et al. sowie von Storm et al. sehen in der Hautwiderstandmessung ein effektives Verfahren zur Stressprävention und zur Narkosetiefemessung (Storm et al. 2002, Winterhalter 2007). Zudem gibt es Untersuchungen, die in der Hautwiderstandsmessung eine Möglichkeit zur Objektivierung des Schmerzempfindens von pädiatrischen Patienten sehen, die selbst noch keine definitiven Schmerzangaben machen können (Dalal et al. 2012). Im Gegensatz dazu stehen Studien von Lehavi et al. und Kirnö et al. Lehavi und Kollegen konnten nach Anlage eines infraclaviculären Blocks keine signifikanten Änderungen des Hautwiderstandes an der betroffenen Extremität nachweisen (Lehavi et al. 2012). Dieses Ergebnis könnte jedoch auch durch das Vorliegen einer insuffizienten Blockade bedingt sein. Kirnö et al. führten des weiteren bei angelegtem axillären Plexus intraneurale Stimulationen an derselben Extremität durch und konnten damit keine Veränderungen des peripher gemessenen Hautwiderstandes auslösen (Kirnö et al. 1991). In Zukunft bedarf es sicherlich noch weiterer Forschungen, um die Möglichkeiten einer regelhaften klinischen Nutzung der Sympathikographie zu erörtern.

Zusammenfassend konnte in dieser Studie kein Nachweis dafür erbracht werden, dass eine suffiziente Blockade des axillären Plexus eine bessere Stressreduktion für den Patienten bewirkt als eine qualitativ gute Allgemeinanästhesie. Im Verlauf der gesamten Messungen konnten weder ausgeprägte Tachykardien noch massive Blutdruckerhöhungen als Hinweise für perioperativen Stress in einer der beiden Gruppen beobachtet werden. Dies spricht zum einen für die gute Wirkung der Regionalanästhesie, zum anderen aber auch für das gute perioperative Schmerzmanagement. 
Weitere Ursachen für diese Ergebnisse liegen zum einen in der guten Aufklärung der Patienten, die sich individuell für das für sie optimale Narkoseverfahren entschieden haben. Zum anderen ermöglichte der Ablauf dieser Studie, dass nahezu alle Patienten von der Aufklärung bis zur Entlassung durch denselben Anästhesisten betreut werden konnten. Diese direkte Betreuung wäre auch im normalen Klinikalltag wünschenswert, da sie eine deutliche stressreduzierende Wirkung auf die Patienten zu haben scheint, leider ist dies aus organisatorischen Gründen meist nicht möglich.

Einschränkend muss erwähnt werden, dass Patienten, die jünger waren als 18 Jahre oder älter als 80 Jahre, Patienten mit multiplen Vorerkrankungen (ASA > 3), sowie Notfallpatienten und Patienten, bei denen eine Kontraindikation für das ein oder andere Narkoseverfahren bestand, nicht an der Studie teilgenommen haben. Es gab somit keinen Patienten, bei dem das von ihm gewünschte Verfahren nicht durchgeführt werden konnte. Keinem der Patienten musste aufgrund einer absoluten Kontraindikation zu einem bestimmten Verfahren geraten werden. Des Weiteren handelte es sich bei den durchgeführten handchirurgischen Operationen um relativ kleine Eingriffe. So bleibt die Frage offen, wie sich die Parameter bei längeren und risikoreicheren Operationen und bei Patienten mit schweren Vorerkrankungen verhalten hätten. Ferner handelt es sich bei den gemessenen Parametern lediglich um indirekte Stressindikatoren. So sind weitere Studien notwendig, um die hier erhobenen Ergebnisse zu untermauern. Untersuchungen mit dem multimodalen Neuromonitoring, bestehend aus BIS und ESG, und der parallelen Bestimmung der Stresshormone könnten hier weitere wichtige Erkenntnisse liefern. 


\section{Zusammenfassung}

Die Auswahl des individuellen Narkoseverfahrens unter Beachtung der patientenund operationsspezifischen Rahmenbedingungen stellt eine große Herausforderung in der Anästhesie dar. Die Patientensicherheit sowie die Herstellung optimaler Operationsbedingungen spielen dabei eine zentrale Rolle. Studien haben gezeigt, dass die Aktivität des autonomen Nervensystems, die durch verschiedenste perioperative Stressoren beeinflusst wird, einen nicht zu unterschätzenden Faktor für das postoperative Ergebnis darstellt. Es gibt Hinweise, dass eine überschießende traumatisch bedingte Aktivierung des Sympathikus das postoperative Ergebnis nachhaltig beeinflussen und mit einer erhöhten Tumorrezidivrate einhergehen kann (Ben-Eliyahu et al. 1999, Brodner et al. 2011). Zukünftige prospektive Studien sollen diesen Hinweisen weiter nachgehen (Heaney und Buggy 2012). Mit der Regionalanästhesie steht ein effektives Verfahren zur Verfügung, das die überhöhte Sympathikusaktivität effektiv dämpfen kann (Celic-Spuzic 2011). Die bisherigen Studien beziehen sich aber vor allem auf rückenmarksnahe Verfahren und nicht auf periphere Nervenblockaden (Wacker et al. 2005). Ziel dieser Studie war die Beobachtung der Sympathikusaktivität bei handchirurgischen Operationen, mit der Frage, ob eine Regionalanästhesie effektiver vor überschießenden Stressreaktionen schützt als eine Allgemeinanästhesie. Dazu wurden zwei Patientengruppen á 23 Patienten untersucht, die sich entweder unter Allgemeinanästhesie oder axillärer Plexusblockade einer handchirurgischen Operation unterziehen mussten. Die statistische Auswertung der erhobenen Parameter (Herzfrequenz, mittlerer arterieller Druck, Sympathikographie (ESG), Hauttemperatur, Schmerzempfinden (NRS) und Bispectral-Index (BIS)) konnten eine sehr hohe Qualität der durchgeführten Verfahren nachweisen, da sich kein einziger Hinweis auf überschießende sympathische Reaktionen in einer der beiden Gruppen zeigte. Ferner konnte ein signifikant höherer postoperativer Schmerzmittelbedarf, sowie eine längere Aufenthaltsdauer im Aufwachraum auf Seiten der ITN-Gruppe nachgewiesen werden. In der Gruppe der Regionalanästhesien zeigte sich eine zunehmende Auskühlung über die OP-Dauer, was die Bedeutung des perioperativen Wärmeerhalts unterstreicht.

Insgesamt konnte jedoch eine Überlegenheit des regionalen Verfahrens in Bezug auf die Stressprävention nicht nachgewiesen werden. Diese Ergebnisse sollten durch die Untersuchung länger andauernder, risikoreicherer Operationen und Anästhesien, anderer regionaler Verfahren und die intraoperative Bestimmung von Stresshormonen weiter untersucht werden. 


\section{Anhang}

Einverständniserklärung

UNIVERSITÄTSMEDIZIN GÖTTINGEN

\section{UMG}

Studienleiter: $\quad$ Priv.-Doz. Dr. med. José Hinz

Zentrum Anaesthesiologie, Rettungs- und Intensivmedizin

Universitätsmedizin Göttingen

Robert-Koch-Str. 40

37075 Göttingen Tel: 0551-3912639

Studienärzte: $\quad$ Dr. med. Albert Götte

Klinik für Anästhesiologie, Intensivmedizin und Schmerztherapie

St. Vincenz - Krankenhaus Paderborn

Am Busdorf 2

33098 Paderborn

Tel:05251-861585

Einverständniserklärung zur Untersuchung

„Beobachtung der Sympathikusaktivität bei handchirurgischen Operationen unter Plexusblockade oder Allgemeinanästhesie“

Ich, geb. am wurde von dem

aufklärenden Arzt vollständig über Wesen, Bedeutung und Tragweite der Untersuchung mit dem o.g. Titel aufgeklärt. Ich habe den Aufklärungstext gelesen und verstanden. Ich hatte die Möglichkeit, Fragen zu stellen, und habe die Antworten verstanden und akzeptiere sie. Der Arzt hat mich über die mit der Teilnahme an der Untersuchung verbundenen Maßnahmen und Risiken informiert.

Ich hatte ausreichend Zeit, mich zur Teilnahme an dieser Studie zu entscheiden und weiß, dass die Teilnahme an dieser Untersuchung freiwillig ist. Ich weiß, dass ich jederzeit und ohne Angaben von Gründen diese Zustimmung widerrufen kann.

Ich habe dem aufklärenden Arzt vollständige und wahrhafte Angaben über meinen Gesundheitszustand, über Vorerkrankungen und über einen etwaigen Konsum von Alkohol, Nikotin oder Drogen gemacht. Ich versichere, dass keine der Ausschlusskriterien auf mich zutreffen.

Mir ist bekannt, dass meine persönlichen Daten in verschlüsselter (pseudonymisierter) Form gespeichert und für wissenschaftliche Publikationen verwendet werden.

Mit meiner Einwilligung erkläre ich mich damit einverstanden, dass meine persönlichen Daten, die erhobenen Daten der Stressmessung und die weiteren Werte wie Herzfrequenz, Blutdruck, Körpertemperatur und Sauerstoffsättigung im Blut sowie die relevanten Daten aus meiner Krankenakte für die Dauer von 10 Jahren gespeichert und 
in anonymisierter Form für wissenschaftliche Publikationen verwendet werden. Es erfolgt keine Weitergabe meiner Daten an Dritte.

Ich habe das Recht, diese Einwilligung jeder Zeit zu widerrufen. Die gespeicherten persönlichen Daten werden auf Verlangen gelöscht.

Ich habe eine Kopie der Patienteninformation und dieser Einwilligungserklärung erhalten. Ich erkläre hiermit die freiwillige Teilnahme an dieser Untersuchung.

Ort und Datum

Ort und Datum
Unterschrift des/der Probanden/in

Unterschrift des aufklärenden Arztes 


\title{
Patientenaufklärung
}

\section{UNIVERSITÄTSMEDIZIN \\ comonex: UMG}

\author{
Studienleiter: $\quad$ Priv.-Doz. Dr. med. José Hinz \\ Zentrum Anaesthesiologie, Rettungs- und Intensivmedizin \\ Universitätsmedizin Göttingen \\ Robert-Koch-Str. 40 \\ 37075 Göttingen Tel: 0551-3912639 \\ Studienärzte: $\quad$ Dr. med. Albert Götte \\ Klinik für Anästhesiologie, Intensivmedizin und Schmerztherapie \\ St. Vincenz - Krankenhaus Paderborn \\ Am Busdorf 2 \\ 33098 Paderborn
}

Tel:05251-861585

\section{Patientenaufklärung zur Studie}

\section{„Beobachtung der Sympathikusaktivität bei handchirurgischen Operationen unter Plexusblockade oder Allgemeinanästhesie“"}

Sehr geehrte Patientin, sehr geehrter Patient,

wir bitten Sie um die freiwillige Teilnahme an einer Untersuchung mit der wir den Einfluss der Narkoseform auf den Stress des Patienten messen. Der genaue Ablauf und die Methoden sind unten näher beschrieben.

Der Körper des Menschen kann sich unabhängig von dessen Willen an bestimmte Situationen anpassen. Man bemerkt zum Beispiel in Stresssituationen, dass das Herz schneller schlägt und dass man feuchte Hände bekommt, ohne dies beeinflussen zu können.

Diese Vorgänge werden über das so genannte vegetative oder autonome Nervensystem gesteuert, das der Mensch nicht willentlich beeinflussen kann.

Eine Operation ist eine Stresssituation für den Körper.

Auch wenn der Patient während der Operation schläft reagiert das autonome Nervensystem auf diesen Stress. Ziel einer jeden Narkose ist es, diesen Stress möglichst gering zu halten. Um diesem Ziel näher zu kommen, möchten wir den Stress unter Vollnarkose und bei „örtlicher“ Betäubung vergleichen.

Sie haben keinen direkten Nutzen von der Teilnahme an dieser Studie.

Mit Hilfe eines besonderen Gerätes, des Elektrosympathikographen, ist es uns möglich den Stress eines Patienten indirekt zu messen und damit Rückschlüsse auf die Aktivität des autonomen Nervensystems zu ziehen.

Um diese Messung durchzuführen, werden an Ihrem Daumen und an Ihrem Zeigefinger zwei Klebeelektroden angebracht. Diese Elektroden kennen Sie von einer EKG-Untersuchung. 
An diesen Elektroden liegt ein minimaler Strom an, der für Sie weder spürbar noch gefährlich ist. Unser Gerät misst nun den Widerstand der Haut zwischen den beiden Elektroden.

Wenn der Körper bei einer Operation Stress erfährt, so kommt es zu einer erhöhten Aktivität des autonomen Nervensystems und kleine Drüsen in der Haut produzieren vermehrt Schweiß. Die Hände werden feucht. Diese Feuchtigkeit führt zu einer besseren Leitung des Stroms und damit zu einem geringeren Hautwiderstand.

Je größer der Stress des Körpers, desto feuchter wird die Haut. Der Hautwiderstand sinkt.

Die Messung erfolgt vor, während und nach der Operation. Zudem werden durch unsere Narkosegräte noch Ihre Herzfrequenz, Ihr Blutdruck, und der Sauerstoffgehalt in Ihrem Blut erfasst. Im Abstand von fünf Minuten wird weiterhin Ihre Körpertemperatur mit einem Thermometer gemessen.

Diese Werte werden unabhängig von dieser Studie bei jeder Narkose erfasst, lediglich die Messung der Körpertemperatur und die Stressmessung erfolgt studienbedingt.

Sollte vor der Operation eine Blutentnahme erfolgen, so hat diese nichts mit der Studie zu tun.

Ferner werden wir Einblick in Ihre Krankenakte nehmen, um zu sehen, ob es Dinge gibt, die sich auf den Stress Ihres Körpers auswirken könnten.

Alle erfassten Werte werden von uns notiert und später ausgewertet.

Bei unserer Untersuchung möchten wir vergleichen, ob der Körper während einer Operation unter Vollnarkose oder bei einer „örtlichen“ Betäubung mehr Stress erfährt.

Es steht Ihnen frei sich für eine der beiden Narkoseformen zu entscheiden.

Die Teilnahme an dieser Untersuchung ist mit keinem Risiko verbunden. Eine Verletzung durch den Elektrosympathikographen ist ausgeschlossen.

Die Teilnahme an dieser Untersuchung ist freiwillig. Sie können die Teilnahme an der Untersuchung zu jeder Zeit ohne Angabe von Gründen beenden. Mit Ihrer Unterschrift willigen Sie in die Erhebung, Speicherung und wissenschaftliche Auswertung der o.g. Daten ein. Die erhobenen Daten werden in pseudonymisierter Form gespeichert, d.h. mit einer Codenummer versehen, die den Rückschluss auf Ihre Person unmöglich macht. Die Daten werden für die gesetzlich vorgeschriebene Dauer von zehn Jahren gespeichert. Es erfolgt keine Weitergabe der Daten an Dritte. Sie können diese Einwilligung widerrufen und verlangen, dass Ihre persönlichen Daten gelöscht werden.

Bei Rückfragen können Sie sich an den Leiter der Untersuchung wenden. Die Telefonnummer finden Sie in der Kopfzeile. 
Überwachungsprotokoll

Überwachungsprotokoll für Sympathikus-ESG-Allgemeinanästhesie/ Plexusanästhesie Untersuchung:

lfd. Nummer.:

Ort:

Geräte:

Raumtemperatur:

Analgetikabedarf intraoperativ:

Analgetikabedarf postoperativ :

Vorerkrankungen :

Vormedikation:

Anästhesieform: Allgemeinanästhesie Plexusanästhesie

OP/Untersuchungsdatum:

Untersucher:

Operateur: 
Datum:

\section{Uhrzeit}

(h/min/sec) 1fd. Nummer:

ESG

(kOhm)

(mmHG)

$(1 / \mathrm{min})$

(mmHg)

(\%)

Seitenzahl:

\begin{tabular}{|c|c|c} 
BIS & $T$ & Sevo
\end{tabular}

$\left({ }^{\circ} \mathrm{C}\right)$ (MAC)

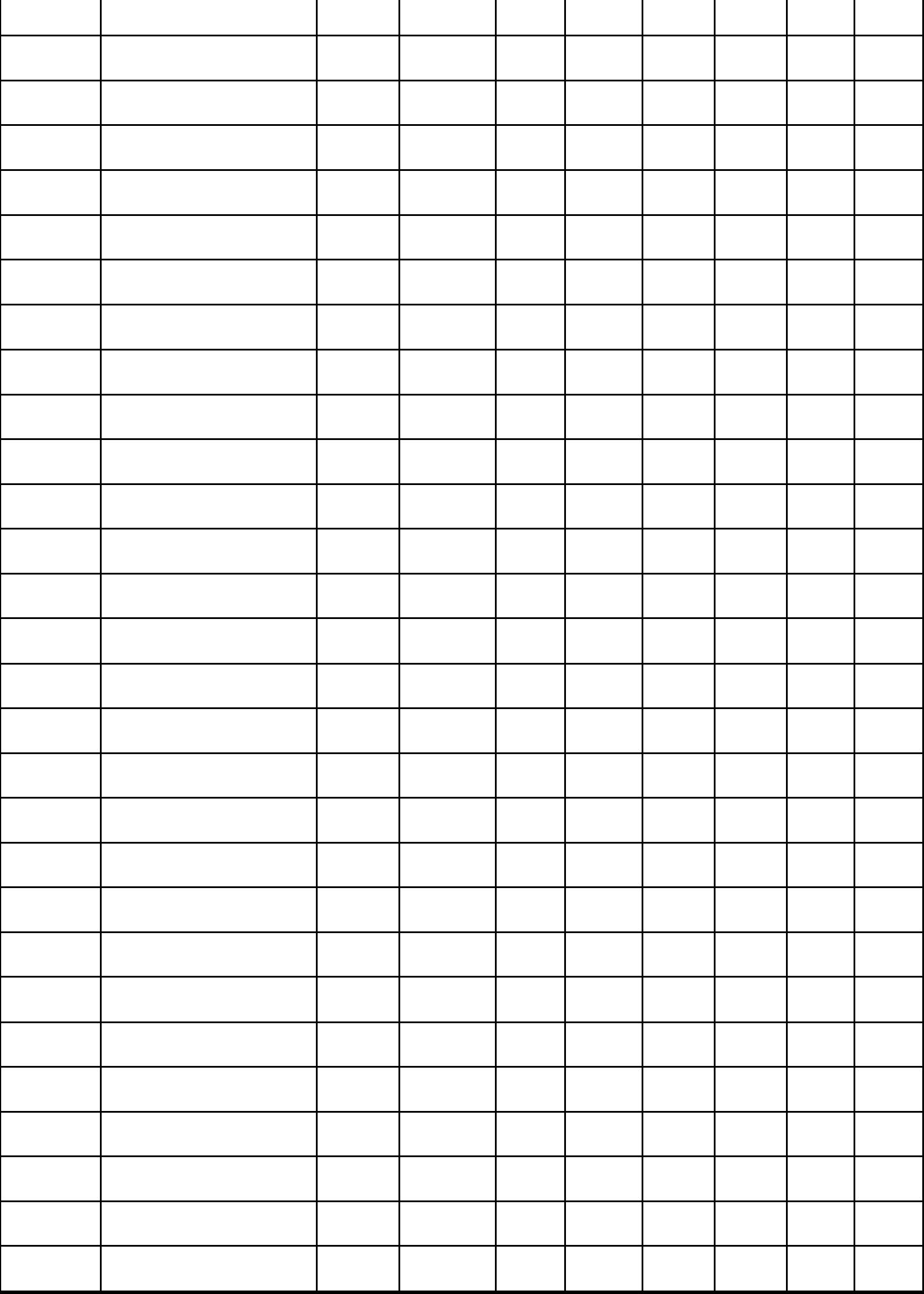


Besonderheiten?

(Zahnschäden, PONV, Hypotension (RR-Abfall<20\%)

Komplikationen bei Plexusanlage?

Postoperative Messung:

NRS (0-10)

\begin{tabular}{|l|l|l|l|}
\hline & direkt post OP & 6h post OP & 1Tag post OP \\
\hline Uhrzeit & & & \\
\hline NRS & & & \\
\hline
\end{tabular}

Zufriedenheit in Noten (1-6): 


\section{Tabellenverzeichnis}

Tabelle 1: $\quad$ Erläuterung der BIS-Werte (ab Version 3.0)

Tabelle 2: $\quad$ Übersicht der Messzeitpunkte

Tabelle 3: Analyse der anthropometrischen Daten

Tabelle 4: peri- und postoperative Schmerzen

Tabelle 5: intra- und postoperativer Schmerzmittelbedarf

Tabelle 6: statistische Auswertung der gesamten Messung 


\section{Abbildungsverzeichnis}

Abbildung 1: Basismonitoring im Aufwachraum

Abbildung 2: Anschluss des ESG

Abbildung 3: $\quad$ Tischgerät ESG 1001

Abbildung 4: $\quad$ zeitlicher Verlauf der Veränderungen der Herzfrequenz in beiden Gruppen über die gesamte Messung

Abbildung 5: $\quad$ zeitlicher Verlauf der prozentualen Änderung der Herzfrequenz in beiden Gruppen über die gesamte Messung

Abbildung 6: zeitlicher Verlauf der absoluten Werte des MAD in beiden Gruppen über die gesamte Messung

Abbildung 7: $\quad$ zeitlicher Verlauf der prozentualen Änderung des MAD in beiden Gruppen über die gesamte Messung

Abbildung 8: zeitlicher Verlauf der Hautwiderstandswerte in beiden Gruppen über die gesamte Messung

Abbildung 9: zeitlicher Verlauf der prozentualen Änderung des Hautwiderstands in beiden Gruppen über die gesamte Messung

Abbildung 10: zeitlicher Verlauf der Hauttemperatur in beiden Gruppen über die gesamte Messung

Abbildung 11: zeitlicher Verlauf der Abweichung der Hauttemperatur vom Ausgangswert in beiden Gruppen 


\section{Literaturverzeichnis}

Adams HA, Biscoping J, Baumann P, Börmann A, Hempelmann G (1989): Mütterliche und kindliche Streß-Parameter bei Schnittentbindungen in Allgemein- und Periduralanaesthesie. Reg Anaesth 12, 87 - 94

Ahlers O, Nachtigall I, Lenze J, Goldmann A, Schulte E, Höhne C, Fritz G, Keh D (2008): Intraoperative thoracic epidural anaesthesia attenuates stress-induced immunosuppression in patients undergoing major abdominal surgery. Br J Anaesth $\underline{101}, 781-787$

Anand KJS, Hickey PR (1992): Halothane-morphine compared with high-dose sufentanil for anesthesia and postoperative analgesia in neonatal cardiac surgery. N Engl J Med $\underline{326}$, $1-9$

Añez C, Papaceit J, Sala JM, Fuentes A, Rull M (2001): The effect of encephalogram bispectral index monitoring during total intravenous anesthesia with propofol in outpatient surgery. Rev Esp Anestesiol Reanim 4, 264 - 269

Avidan MS, Jacobsohn E, Glick D, Burnside BA, Zhang L, Villafranca A, Karl L, Kamal S, Torres B, O’Connor M, Evers AS, Gradwohl S, Lin N, Palanca BJ, Mashour GA (2011): Prevention of intraoperative awareness in a high-risk surgical population. $\mathrm{N}$ Engl J Med 365, 591 - 600

Becker R: Interindividuelle Auswirkungen von Schlafentzug. Phil. Diss. Berlin 2008

Ben-Eliyahu S, Page GG, Yirmiya R, Shakhar G (1999): Evidence that stress and surgical interventions promote tumor development by suppressing natural killer cell activity. Int J Cancer $\underline{80}, 880$ - 888

Berger H (1929): Über das Enzephalogramm des Menschen. Arch Psychiatr Nervenkr $\underline{\text { 77, }} 527$ $-570$

Bergmann F, Dmoch N, Farber MU, Grüll K, Ihne W, Kreß K, Lottermoser J, Lübke W, Salm W, Zöllner W: Grundgesetze des elektrischen Stromes; in: Einführung in die Physik, Ausgabe E; hrsg. v. Ihne W, Lottermoser J, Zöllner W; Verlag Moritz Diesterweg, Frankfurt am Main 1994, 50 - 73

Bergmann I, Göhner A, Crozier TA, Hesjedal B, Wiese CH, Popov AF, Bauer M, Hinz JM (2012): Surgical pleth index-guided remifentanil administration reduces remifentanil and propofol consumption and shortens recovery times in outpatient anaesthesia. $\mathrm{Br} \mathbf{J}$ Anaesth

Birbaumer N, Schmidt RF: Allgemeine Physiologie der Großhirnrinde; in: Physiologie des Menschen, Band 28; hrsg. v. Schmidt RF, Thews G, Lang F; Springer Verlag, Berlin u.a. $2000,128-140$ 
Böttner C: Erkrankungen der Hand; in: Facharztkompendium Orthopädie und Unfallchirurgie, Band 4; hrsg. v. Böttner C; OrthoForum-Verlag, Berlin 2010, 641 - 658

Brockmann C, Raasch C, Bastian C (2000): Verhalten endokriner Stressparameter bei remifentanil- oder sufentanilgestützter TIVA. Anästhesiol Intensivmed Notfallmed Schmerzther $\underline{35}, 685$ - 691

Brodner G, Van Aken H, Gottschalk A: Die thorakale Periduralanalgesie - ist eine Neubewertung notwendig? in: Refresher Course Aktuelles Wissen für Anästhesisten, Nr. 37; hrsg. v. der Deutschen Akademie für Anästhesiologische Fortbildung; Aktiv Druck \& Verlag GmbH, Ebelsbach 2011, 49 - 70

Bromm B: Neurophysiologie des nozizeptiven Systems; in: ains, Band 4: Schmerztherapie; hrsg. v. Beck H, Martin E, Motsch J, Schulte am Esch J; Georg Thieme Verlag, Stuttgart u.a. 2002, 29 - 41

Bruhn J (2003): Vergleich verschiedener EEG Parameter: Spektrale Eckfrequenz 95, approximale Entropie und Bispektral-Index. Anästh Intensivmed 44, 17 - 21

Burk CL: Phasische elektrodermale Aktivität als Persönlichkeitsindikator. Phil. Diss. Gießen 2005

Busse M: Gefäßsystem und Kreislaufregulation; in: Physiologie des Menschen, Band 28; hrsg. v. Schmidt RF, Thews G, Lang F; Springer Verlag, Berlin u.a. 2000, 498 - 560

Celic-Spuzic E (2011): Effect of anesthesia on the changes in the hormones levels during and after transvesical prostatectomy. Med Arh 65, 348 - 353

Chen X, Thee C, Gruenewald M, Wnent J, Illies C, Hoecker J, Hanss R, Steinfath M, Bein B (2010): Comparision of surgical stress index-guided analgesia with standard clinical practice during routine general anesthesia: a pilot study. Anesthesiology 112, 1175 1183

Chen X, Thee C, Gruenewald M, Ilies C, Höcker J, Hanss R, Steinfath M, Berin B (2012): Correlation of surgical pleth index with stress hormones during propofol-remifentanil anaesthesia. Sci World J 2012, 1 - 8

Chizmadzhev YA, Indenbom AV, Kuzmin PI, Galichenko SV, Weaver JC, Potts RO (1998): Electrical properties of skin at moderate voltages: contribution of appendageal macropores. Biophys J $\underline{74}, 843$ - 856

Dalal PG, Doheny KK, Klick L, Britcher S, Rebstock S, Benzinover D, Palmer C, Berlin C, Postula M, Kong L, Janicki PK (2012): Analysis of acute pain scores and skin conductance measurements in infants. Early Hum Dev 12, S0378 - 3782

Darrow CW (1927): Sensory, secretory, and electrical changes in the skin following bodily excitation. J Exp Psychol 10, 197 - 226 
Dawson ME, Schell AM, Filion, DL: The electrodermal system; in: Handbook of Psychophysiology, $2^{\text {nd }}$ ed.; hrsg. v. Cacioppo JT, Tassinary L G, Berntson GG; University Press, Cambridge 2000, 200 - 223

Domino KB, Posner KL, Caplan RA, Cheney FW (1999): Awareness during anaesthesia: a closed claim analysis. Anesthesiology 90, 1053 - 1061

Elenkov IJ, Ronald LW, George PC, Sylvester EV (2000): The sympathetic nerve - an integrative interface between two supersystems: the brain and the immune system. Pharmacol Rev 52(4), 595 - 638

Evans JM: Clinical signs and automatic responses; in: Consciousness, awareness and pain in general anaesthesia; hrsg. v. Rosen M, Lunn JN; Butterworth, London 1987, 18 - 34

Féré C (1888): Note sur les modifications de la résistance électrique sous l'influence des excitations sensorielles et des émotions. CR Soc Biol 모, 217 - 219

Freye E, Grabitz K, Latasch L (2000): Bestimmung der analgetischen Tiefe während der Anästhesie. Anästh Intensivmed 41, 71 - 81

Glass PS, Blom M, Kearse L, Rosow C, Sebe P, Manberg P (1997): Bispectral analysis measures sedation and memory effects of propofol, midazolam, isoflurane, and alfentanil in healthy volunteers. Anesthesiology $\underline{86}, 836$ - 847

Götte A: TIVA und autonomes Nervensystem. Zitiert nach vorliegendem Vortrag auf: Internationales Symposium für Anästhesie-, Notfall-, Schmerz- und Intensivbehandlungsprobleme, St. Anton am Arlberg, 30. Januar bis 06. Februar 1999, o. Hrsg., o. Verl., o.O.

Gratzl M: Haut; in: Histologie; hrsg. v. Junqueira LC, Carneiro J, Kelley RO; Springer Verlag Berlin u.a. 2002, 311 - 324

Hach-Wunderle V: Gefäße; in: Basislehrbuch Innere Medizin; hrsg. v. Renz-Polster H, Krautzig S, Braun J; Urban\&Fischer-Verlag, München u.a. 2004, 193 - 254

Heaney Á, Buggy DJ (2012): Can anaesthetic and analgesic techniques affect cancer recurrence or metastasis? Br J Anaesth $\underline{109}, 17$ - 28

Herwig R., Brinkmann OA, Sievert KD, Brodner G, Hertle L (2003): Cystectomy causes immunosuppression in bladder cancer. Adv Exp Med Biol 539(Pt B), 741 - 748

Hick A: Vegetatives Nervensystem; in: Kurzlehrbuch Physiologie; hrsg. v. Hick C, Hick A; Urban\&Fischer-Verlag, München u.a. 2000, 257 - 273

Holst, DV (1993): Zoologische Stress-Forschung - ein Bindeglied zwischen Psychologie und Medizin. Spektrum Wiss $\underline{5}, 92-96$

Huiku M, Uutela K, van Gils M, Korhonen I, Kymäläinen M, Meriläinen P, Paloheimo M, Rantanen M, Takala P, Viertiö-Oja H, Yli-Hankala A (2007): Assessment of surgical stress during general anaesthesia. Br J Anaesth $\underline{98}, 447$ - 455 
Iselin-Chaves IA, Flaishon R, Sebel PS, Howell S, Gan TJ, Sigl J, Ginsberg B, Glassm PS (1998): The effect of the interaction of propofol and alfentanil on recall, loss of consciousness, and the Bispectral Index. Anesth Analg 87, 949 - 955

Jänig W: Vegetatives Nervensystem; in: Physiologie des Menschen; hrsg. v. Schmidt RF, Thews G, Lang F; Springer Verlag, Berlin u.a. 2000, 340 - 369

Janitzki A: Funktionsweise des ESG 1001 Monitor-Systems; in: symPATI-GRAPH ESG 1001 Monitorsystem Gebrauchsanweisung; hrsg. v. Janitzki A. o.Verl., o.O. 2010a, 41

Janitzki A: Technische Daten; in: symPATI-GRAPH ESG 1001 Monitorsystem Gebrauchsanweisung; hrsg. v. Janitzki A. o.Verl., o.O. 2010b, 7-1 - 7-2

Janitzki A, Götte A (1995): Spinal anesthesia and functional sympathetic nerve block. Anaesthesist 44, $171-177$

Kahle W: Vegetatives Nervensystem; in: Taschenatlas der Anatomie, Band 3: Nervensystem und Sinnesorgane; hrsg. v. Kahle W, Frotscher M; Georg Thieme Verlag, Stuttgart u.a. 2001,292

Kalia YN, Guy RH (1995): The electrical characteristics of human skin in vivo. Pharm Res $\underline{12}, 1605-1613$

Katoh K, Suzuki A, Ikeda K (1998): Electroencephalographic derivatives as a tool for predicting the depth of sedation and anesthesia induced by sevoflurane. Anesthesiology $\underline{88}, 642$ - 650

Kawagoe I, Tajima K, Kanai M, Kimura S, Mitsuhata H (2011): Comparison of intraoperative stress hormones release between propofol-remifentanil anesthesia and propofol with epidural anesthesia during gynecological surgery. Masui. Jpn J Anesthesiol $\underline{60}, 416$ 424

Kirnö K, Kunimito M, Lundin S, Elam M, Wallin BG (1991): Can galvanic skin response be used as a quantitative estimate of sympathetic nerve activity in regional anesthesia. Anesth Analg 73, 138 - 142

Knabe D, Götte A, Janitzki A: Sympathovegetative Reaktionen, Kreislaufeffekte und Schmerzen postoperativ nach Regionalanästhesien. Zitiert nach vorliegendem Poster in: Deutscher Anästhesie Kongress, Hamburg, 22. April bis 26. April 1997, o. Hrsg., o. Verl., o.O.

Larsen R: Kardiovaskuläre Medikamente; in: Anästhesie; hrsg. v. Larsen R; Urban\&Fischer. München u.a. 2010a, 7 - 188

Larsen R: Narkosetiefe bei Inhalationsanästhesien; in: Anästhesie; hrsg. v. Larsen R; Urban\&Fischer. München u.a. 2010b, 31 - 33

Lehavi A, Kiorescu A, Abecasis P, Baskevitsch A, Katz Y (2012): Skin electrical resistance does not change following infraclavicular block. AANA J $\underline{80}, 185$ - 188 
Lehmann A, Thaler E, Boldt J (2001): Ist es sinnvoll, die Narkosetiefe zu messen? - Ein Versuch der Marktübersicht über die kommerziell erhältlichen Geräte zur Messung

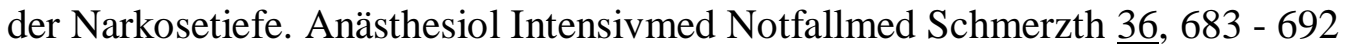

Lidberg L, Wallin BG (1981): Sympathetic skin nerve discharges in relation to amplitude of skin resistance responses. Psychophysiology 18, 268 - 270

Lipfert P, Arndt JO (1993): Kreislaufeffekte rückenmarksnaher Leitungsanästhesien. Anaesthesist 42, 773 - 787

Liu J, Singh H, White PF (1996): Electroencephalogram bispectral analysis predicts the depth of midazolam-induced sedation. Anesthesiology $\underline{84}, 64$ - 69

Liu J, Singh H, White PF (1997): Electroencephalographic bispectral index correlates with intraoperative recall and depth of propofol-induced sedation. Anesth Analg $\underline{84}, 185$ 189

Lorke D: Schmerzrelevante Neuroanatomie; in: ains, Band 4: Schmerztherapie; hrsg. v. Beck H, Martin E, Motsch J, Schulte am Esch J; Georg Thieme Verlag, Stuttgart u.a. 2002, $13-38$

Ludwigs JA: Evaluierung des surgical stress index bei Allgemein- und Spinalanästhesien. Med. Diss. Kiel 2011

Macefield VG, Elam M, Wallin BG (2002): Firing properties of single postganglionic sympathetic neurones recorded in awake human subjects. Auton Neurosci 95(1-2), $146-159$

Mast J: Nervus medianus evozierte Potentiale und Bispektralindex während wiederholten Phasen von Wachheit und Bewusstlosigkeit. Med. Diss. Berlin 2009

Ogawa T (1975): Thermal influence on palmar sweating an mental influence on generalized sweating in man. Jpn J Surg 25, 525 - 536

Rampil IJ (1998): A primer for EEG signal processing in anesthesia. Anesthesiology 90, 890 1002

Rudigier J: Nervenkompressionssyndrome; in: Kurzgefasste Handchirurgie; hrsg. v. Rudigier J; Thieme Verlag, Stuttgart u.a. 2006, 304 - 327

Sato K, Sato F (1983): Individual variations in structure and function of human eccrine sweat gland. Am J Physiol 245(2), 203 - 208

Sato K, Kang WH, Saga K, Sato KT (1989): Biology of sweat glands and their disorders. J Am Acad Dermatol 20, 537 - 563

Schaible HG and Schmidt RF: Nozizeption und Schmerz; in: Physiologie des Menschen, Band 28; hrsg. v. Schmidt RF, Thews G, and Lang F; Springer Verlag, Berlin u.a. $2000,236-250$ 
Schiebler TH, Schmidt W, Zilles K: Hautanhangsorgane; in: Anatomie; hrsg. v. Schiebler TH, Schmidt W, Zilles K; Springer Verlag, Berlin u.a. 1999a, 212 - 218

Schiebler TH, Schmidt W, Zilles K: Vegetatives Nervensystem; in: Anatomie; hrsg. v. Schiebler TH, Schmidt W, Zilles K; Springer Verlag, Berlin u.a. 1999b, 197 - 203

Schiebler TH, Schmidt W, Zilles K: Zentralnervensystem; in: Anatomie; hrsg. v. Schiebler TH, Schmidt W, Zilles K; Springer Verlag, Berlin u.a. 1999c, 719 - 834

Schlereth T, Dieterich M, Birklein F (2009): Hyperhidrose - Ursachen und Therapie von übermäßigem Schwitzen. Dtsch Arztebl Int 106(3), 32 - 37

Schmidt GN, Müller J, Bischoff P (2008): Messung der Narkosetiefe. Anaesthesist 57, 9 - 35

Simon, E. Wärmehaushalt und Temperaturregelung; in: Physiologie des Menschen; hrsg. v. Schmidt RF, Thews G, Lang F; Springer Verlag, Berlin u.a. 2000, 640 - 671

Song D, Joshi GP, White PF (1997): Titration of volatile anesthetics using bispectral index

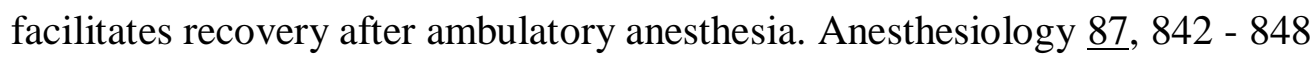

Storm H, Myre K, Rostrup M, Stokland O, Lien MD, Raeder JC (2002): Skin conductance correlates with perioperative stress. Acta Anaesthesiol Scand 46, 887 - 895

Storm H (2008): Changes in skin conductance as a tool to monitor nociceptive stimulation and pain. Curr Opin Anaesthesiol 21(6), 796 - 804

Struys MMRF, Vanpeteghem C, Huiku M, Uutela K, Blyaert NBK, Mortier EP (2007): Changes in a surgical stress index in response to standardized pain stimuli during propofol - remifentanil infusion. Br J Anaesth 99, 359 - 367

Tarchanoff JD (1890): Ueber die galvanischen Erscheinungen in der Haut des Menschen bei Reizungen der Sinnesorgane und bei verschiedenen Formen der psychischen Tätigkeit. Pflugers Arch 46(1), 46 - 55

Tolksdorf W: Der präoperative Streß; in Der präoperative Streß; hrsg. v. Tolksdorf W; Springer Verlag, Berlin u.a. 1985, 1 - 4

Tsigos C, Chrousos GP (2002): Hypothalamic-pituitary-adrenal axis, neuroendocrine factors and stress. J Psychosom Res 53, 865 - 871

Wacker J, Pasch T, Schaub MC, Zaugg M (2005): Perioperative Strategien zur Regulierung des Sympathikotonus. Anaesthesist 54, 303 - 318

Wallenborn J (2012): Neuromonitoring in der Anästhesiologie. Anästh Intensivmed $\underline{53}$, 151 167

Wilhelm W, Kreuer S (2003): Das interpretierte EEG als Überwachungsverfahren in der Anästhesiologie. Anästh Intensivmed 44, 8 - 15 
Winterhalter M: Messungen der Hautimpedanz: Ein neuer Weg zur "Narkosetiefebestimmung" in der Allgemeinanästhesie Med. Habil.-Schr. Hannover 2007

Winterhalter M, Schiller J, Münte S, Bund M, Hoy L, Weilbach C, Piepenbrock S, RaheMeyer N (2007a): Prospective investigation into the influence of various stressors on skin impedance. J Clin Monit Comput 22, 67 - 74

Winterhalter M, Adams HA, Engels T, Rahe-Meyer N, Zuk J, Hagl C, Hecker H, Piepenbrock S (2007b): Endocrine stress response and myocardiac outcome under balanced anesthesia with sevoflurane or TIVA with propofol in patients undergoing CABG. Intensivmed Notfallmed $\underline{44}, 166$ - 177

Winterhalter M, Münte S, Gerhard M, Danzeisen O, Jütter T, Monaca E, Hoy L, Rahe-Meyer N, Kienbaum P (2010): Prospective study comparing skin impedance with EEG parameters during the induction of anaesthesia with fentanyl and etomidate. Eur J Med Res 15, 47 - 53

Wolf AR (2012): Effects of regional analgesia on stress responses to pediatric surgery. Paediatr Anaesth 22, 19 - 24

Wuttke W: Endokrinologie; in: Physiologie des Menschen; hrsg. v. Schmidt RF, Thews G, Lang F; Springer Verlag, Berlin u.a. 2000, 370 - 407

Yamamoto T, Yamamoto Y (1976): Electrical properties of the epidermal stratum corneum. Med Biol Eng 14, 151 - 158

Zaps D: Elektro-physiologische Untersuchungen über den Einfluss chirurgischer Eingriffe auf den elektrischen Hautwiderstand im Bereich von Akupunkturpunkten. Med. Diss. München 2010 


\section{Danksagung}

Ich bedanke mich bei Herrn Prof. Dr. José Hinz, der mir während der gesamten Dissertation jederzeit mit Rat und Tat sowie außerordentlichem persönlichen Engagement zur Seite stand. Ich bedanke mich für die Überlassung dieses interessanten Themas, für die Unterstützung bei der statistischen und graphischen Auswertung und für die Durchsicht der Arbeit.

Mein Dank gilt Herrn Dr. Albert Götte, der mir mit seiner Beharrlichkeit und seinem Enthusiasmus während der gesamten Entstehung der Dissertation helfend zur Seite stand. Ich bedanke mich für das entgegengebrachte Vertrauen, die Bereitstellung der notwendigen Geräte und für die Möglichkeit, die Datenerhebung parallel zu meiner beruflichen Tätigkeit durchführen zu können.

Ich danke Herrn Prof. Dr. Dr. Martin Bauer und Herrn Dr. Martin Baur für ihre Unterstützung und Hilfsbereitschaft während der gesamten Dissertation.

Herrn Prof. Dr. Dr. Thomas Crozier danke ich für die Unterstützung bei der Erstellung des Ethikantrages.

Ich bedanke mich bei Herrn Dr. Armin Janitzki für die Bereitstellung des Elektrosympathikographen und für die stetige Unterstützung bei allen praktischen und theoretischen Fragen in Bezug auf die Sympathikographie.

Frau Felicitas Reinke und Herrn Dr. Stephan Blank danke ich für ihr Verständnis und ihre Geduld, mit der sie mir die Datenerhebung in der handchirurgischen Abteilung des St. Vincenz Krankenhauses ermöglicht haben.

Den ärztlichen und pflegerischen Kolleginnen und Kollegen der Abteilung für Anästhesiologie, Intensivmedizin und Schmerztherapie des St. Vincenz Krankenhauses Paderborn gilt mein besonderer Dank für ihre großartige Unterstützung. 


\section{Lebenslauf}

Mein Name ist Andreas Manfred Klaholz. Ich wurde am 16.11.1980 in Brilon geboren. Ich bin der erste Sohn des Diplom-Vermessungsingenieurs Hubert Klaholz und der pharmazeutisch-technischen Assistentin Maria Klaholz (geb. Erlemeier). Von 1987 bis 1991 besuchte ich die St. Engelbert Grundschule in Brilon. Anschließend besuchte ich das Gymnasium Petrinum in Brilon und erwarb dort im Juni 2000 die Allgemeine Hochschulreife. Vom Juli 2000 bis zum April 2001 leistete ich meinen Grundwehrdienst als Transportsoldat des Transportbataillons 801 in Lippstadt. Im April 2001 begann ich mein Studium der Humanmedizin an der Rheinischen Friedrich-Wilhelms-Universität in Bonn. Dort beendete ich im September 2003 das vorklinische Studium mit dem Erwerb des Physikums. Vom Oktober 2003 bis zum November 2007 studierte ich an der Universität zu Lübeck. Im November 2007 beendete ich mit dem Bestehen des Staatsexamens das Studium der Humanmedizin und erwarb die Approbation. Neben meinem Studium engagierte ich mich ehrenamtlich als Gruppenleiter der Deutschen Pfadfinderschaft St. Georg. Vom April 2008 bis zum September 2009 arbeitete ich in der Abteilung für Unfallchirurgie des St. Vincenz Krankenhauses in Paderborn. Anschließend arbeitete ich vom Oktober 2009 bis zum März 2010 im Zentrum für Schwerbrandverletzte, Intensiv- und Notfallmedizin des Klinikums Dortmund. Seit April 2010 bin ich als Assistenzarzt in der Abteilung für Anästhesiologie, Intensivmedizin und Schmerztherapie des St. Vincenz Krankenhauses in Paderborn tätig. Neben meiner beruflichen Tätigkeit habe ich die Fachkunde Rettungsdienst erworben und bin seit 2009 im Notarztdienst tätig. Ich bin ausgebildeter Traumamanager und habe den DIVIKurs „Intensivtransport“ absolviert. Meine Dissertationsarbeit habe ich im Juni 2010 unter Leitung von Prof. Dr. J. Hinz in der Abteilung für Anaesthesiologie im Zentrum für Anaesthesiologie, Rettungs- und Intensivmedizin der Universität Göttingen begonnen. 